\title{
TOWARD THE FINITE-TIME BLOWUP OF THE 3D AXISYMMETRIC EULER EQUATIONS: A NUMERICAL INVESTIGATION*
}

\author{
GUO LUO ${ }^{\dagger}$ AND THOMAS Y. HOU $\ddagger$
}

\begin{abstract}
Whether the three-dimensional incompressible Euler equations can develop a singularity in finite time from smooth initial data is one of the most challenging problems in mathematical fluid dynamics. This work attempts to provide an affirmative answer to this long-standing open question from a numerical point of view by presenting a class of potentially singular solutions to the Euler equations computed in axisymmetric geometries. The solutions satisfy a periodic boundary condition along the axial direction and a no-flow boundary condition on the solid wall. The equations are discretized in space using a hybrid 6th-order Galerkin and 6th-order finite difference method on specially designed adaptive (moving) meshes that are dynamically adjusted to the evolving solutions. With a maximum effective resolution of over $\left(3 \times 10^{12}\right)^{2}$ near the point of the singularity, we are able to advance the solution up to $\tau_{2}=0.003505$ and predict a singularity time of $t_{s} \approx 0.0035056$, while achieving a pointwise relative error of $O\left(10^{-4}\right)$ in the vorticity vector $\omega$ and observing a $\left(3 \times 10^{8}\right)$-fold increase in the maximum vorticity $\|\omega\|_{\infty}$. The numerical data are checked against all major blowup/non-blowup criteria, including Beale-Kato-Majda, Constantin-Fefferman-Majda, and Deng-Hou-Yu, to confirm the validity of the singularity. A local analysis near the point of the singularity also suggests the existence of a self-similar blowup in the meridian plane.
\end{abstract}

Key words. 3D axisymmetric Euler equations, finite-time blowup

AMS subject classifications. 35Q31, 76B03, 65M60, 65M06, 65M20

DOI. $10.1137 / 140966411$

1. Introduction. The celebrated three-dimensional (3D) incompressible Euler equations in fluid dynamics describe the motion of ideal incompressible flows in the absence of external forcing. First written down by Leonhard Euler in 1757, these equations have the form

$$
u_{t}+u \cdot \nabla u=-\nabla p, \quad \nabla \cdot u=0,
$$

where $u=\left(u_{1}, u_{2}, u_{3}\right)^{T}$ is the $3 \mathrm{D}$ velocity vector of the fluid and $p$ is the scalar pressure. The $3 \mathrm{D}$ Euler equations have a rich mathematical theory, for which the interested readers may consult the excellent surveys $[2,18,24]$ and the references therein. This paper primarily concerns the existence or nonexistence of globally regular solutions to the 3D Euler equations, which is regarded as one of the most fundamental yet most challenging problems in mathematical fluid dynamics.

The interest in the global regularity or finite-time blowup of (1.1) comes from several directions. Mathematically, the question has remained open for over 250 years and has a close connection to the Clay Millennium Prize Problem on the NavierStokes equations. Physically, the formation of a singularity in inviscid (Euler) flows may signify the onset of turbulence in viscous (Navier-Stokes) flows, and it may

*Received by the editors April 24, 2014; accepted for publication (in revised form) August 15, 2014; published electronically November 18, 2014.

http://www.siam.org/journals/mms/12-4/96641.html

${ }^{\dagger}$ Applied \& Computational Mathematics, California Institute of Technology, MC 9-94, Pasadena, CA 91125. Current address: Department of Mathematics, City University of Hong Kong, Kowloon Tong, Hong Kong (gluo@caltech.edu).

¥Applied \& Computational Mathematics, California Institute of Technology, MC 9-94, Pasadena, CA 91125 (hou@acm.caltech.edu). 
provide a mechanism for energy transfer to small scales. Numerically, the resolution of nearly singular flows requires special numerical treatment, which presents a great challenge to computational fluid dynamicists.

Considerable efforts have been devoted to the study of the regularity properties of the 3D Euler equations. The main difficulty in the analysis lies in the presence of the nonlinear vortex stretching term and the lack of a regularization mechanism, which implies that even the local well-posedness of the equations can only be established for sufficiently smooth initial data (see, for example, [37]). Despite these difficulties, a few important partial results $[3,44,22,46,19,20,25]$ have been obtained over the years which have led to improved understanding of the regularity properties of the 3D Euler. More specifically, the celebrated theorem of Beale, Kato, and Majda [3] and its variants $[22,46]$ characterize the regularity of the 3D Euler equations in terms of the maximum vorticity, asserting that a smooth solution $u$ of (1.1) blows up at $t=T$ if and only if

$$
\int_{0}^{T}\|\omega(\cdot, t)\|_{L^{\infty}} d t=\infty
$$

where $\omega=\nabla \times u$ is the vorticity vector of the fluid. The non-blowup criterion of Constantin, Fefferman, and Majda [19] focuses on the geometric aspects of Euler flows instead and asserts that there can be no blowup if the velocity field $u$ is uniformly bounded and the vorticity direction $\xi=\omega /|\omega|$ is sufficiently "well behaved" near the point of the maximum vorticity. The theorem of Deng, Hou, and $\mathrm{Yu}$ [20] is similar in spirit to the Constantin-Fefferman-Majda criterion but confines the analysis to localized vortex line segments.

Besides the analytical results mentioned above, there also exists a sizable literature focusing on the (numerical) search of a finite-time singularity for the 3D Euler equations. Representative work in this direction include [27, 45], which studied Euler flows with swirls in axisymmetric geometries, the famous computation of Kerr and his collaborators [38, 8, 39], which studied Euler flows generated by a pair of perturbed antiparallel vortex tubes, and the viscous simulations of [5], which studied the 3D Navier-Stokes equations using Kida's high-symmetry initial data. Other interesting pieces of work are [10,47], which studied axisymmetric Euler flows with complex initial data and reported singularities in the complex plane. A more comprehensive list of interesting numerical results can be found in the review article [24].

Although finite-time singularities were frequently reported in numerical simulations of the Euler equations, most such singularities turned out to be either numerical artifacts or false predictions, as a result of either insufficient resolution or inadvertent data analysis procedure (more to follow on this topic in section 4.4). Indeed, by exploiting the analogy between the two-dimensional (2D) Boussinesq equations and the 3D axisymmetric Euler equations away from the symmetry axis, E and Shu [21] studied the potential development of finite-time singularities in the 2D Boussinesq equations, with initial data completely analogous to those of $[27,45]$. They found no evidence for singular solutions, indicating that the "blowups" reported by [27, 45], which were located away from the axis, are likely to be numerical artifacts. Likewise, Hou and Li [32] repeated the computation of [38] with higher resolutions, in an attempt to reproduce the singularity observed in that study. Despite some ambiguity in interpreting the initial data used by [38], they managed to advance the solution up to $t=19$, which is beyond the singularity time $T=18.7$ alleged by [38]. By using newly developed analytic tools based on rescaled vorticity moments, Kerr also confirmed in 
a very recent study [39] that solutions computed from initial data analogous to that used in [38] eventually converge to superexponential growth and hence are unlikely to lead to a singularity. In a later work, Hou and Li [33] also repeated the computation of [5] and found that the singularity reported by [5] is likely an artifact due to insufficient resolution.... To summarize, the existing numerical studies do not seem to provide convincing evidence to support the existence of a finite-time singularity, and the question of whether initially smooth solutions to (1.1) can blow up in finite time remains open.

By focusing on solutions with axial symmetry and other special properties, we have discovered, through careful numerical studies, a class of potentially singular solutions to the 3D axisymmetric Euler equations in a radially bounded, axially periodic cylinder (see (2.1)-(2.2) below). The reduced computational complexity in the cylindrical geometry greatly facilitates the computation of the singularity. With a specially designed adaptive mesh, we are able to achieve a maximum mesh resolution of over $\left(3 \times 10^{12}\right)^{2}$ near the point of the singularity. This allows us to compute the vorticity vector with four digits of accuracy throughout the simulations and to observe a $\left(3 \times 10^{8}\right)$-fold amplification in maximum vorticity. The numerical data are checked against all major blowup/non-blowup criteria, including Beale-Kato-Majda, Constantin-Fefferman-Majda, and Deng-Hou-Yu, to confirm the validity of the singularity. A careful local analysis also suggests the existence of a self-similar blowup in the meridian plane. Our numerical method makes explicit use of the special symmetries built in the blowing-up solutions, which eliminates symmetry-breaking perturbations and facilitates a stable computation of the singularity.

The main features of the potentially singular solutions are summarized as follows. The point of the potential singularity, which is also the point of the maximum vorticity, is always located at the intersection of the solid boundary $r=1$ and the symmetry plane $z=0$. It is a stagnation point of the flow, as a result of the special odd-even symmetries along the axial direction and the no-flow boundary condition (see (2.3)). The vanishing velocity field at this point could have positively contributed to the formation of the singularity, given the potential regularizing effect of convection as observed by $[33,31]$. When viewed in the meridian plane, the point of the potential singularity is a hyperbolic saddle of the flow, where the axial flow along the solid boundary marches toward the symmetry plane $z=0$ and the radial flow marches toward the symmetry axis $r=0$ (see Figure 16(a)). The axial flow brings together vortex lines near the solid boundary $r=1$ and destroys the geometric regularity of the vorticity vector near the symmetry plane $z=0$, violating the ConstantinFefferman-Majda and Deng-Hou-Yu geometric non-blowup criteria and leading to the breakdown of the smooth vorticity field.

The asymptotic scalings of the various quantities involved in the potential finitetime blowup are summarized as follows. Near the predicted singularity time $t_{s}$, the scalar pressure and the velocity field remain uniformly bounded while the maximum vorticity blows up like $O\left(t_{s}-t\right)^{-\gamma}$, where $\gamma$ roughly equals $\frac{5}{2}$. Near the point of the potential singularity, namely the point of the maximum vorticity, the radial and axial components of the vorticity vector grow roughly like $O\left(t_{s}-t\right)^{-5 / 2}$ while the angular vorticity grows like $O\left(t_{s}-t\right)^{-1}$. The nearly singular solution has a locally self-similar structure in the meridian plane near the point of blowup, with a rapidly collapsing support scaling roughly like $O\left(t_{s}-t\right)^{3}$ along both the radial and the axial directions. When viewed in $\mathbb{R}^{3}$, this corresponds to a thin tube on the symmetry plane $z=0$ evolved around the ring $r=1$, where the radius of the tube shrinks to zero as the singularity forms. 
We emphasize that the 3D axisymmetric Euler equations (2.1) are different from their free-space counterpart (1.1) in that they have a constant of motion that is not present in the nonsymmetric case [41]. In addition, it is well known that the choice of the boundary conditions (periodic vs. no-flow) has a nontrivial impact on the qualitative behavior of the solutions of the Euler equations, especially near the solid boundaries $[2,18]$. In view of these differences and the fact that the singularity we discover lies right on the boundary, we stress that the work described in this paper is not directly relevant to the Clay Millennium Prize Problem on the Navier-Stokes equations, which is posed either in free space or on periodic domains. ${ }^{1}$ Rather, it should be viewed as an attempt at the understanding of the effect of solid boundaries in the creation of small scales and, in the case of zero viscosity, the creation of singularities in incompressible flows.

The rest of this paper is devoted to the study of the potential finite-time singularity and is organized as follows. Section 2 contains a brief review of the 3D Euler equations in axisymmetric form and defines the problem to be studied. Section 3 gives a brief description of the numerical method that is used to track and resolve the nearly singular solutions. Section 4 examines the numerical data in great detail and presents evidence supporting the existence of a finite-time singularity. Finally section 5 concludes the paper with a brief discussion on future research directions.

2. Description of the problem. The 3D Euler equations (1.1) with axial symmetry can be conveniently described in the so-called stream-vorticity form. To derive these equations, recall first that in cylindrical coordinates $(r, \theta, z)$, an axisymmetric flow $u$ can be described by the decomposition

$$
u(r, z)=u^{r}(r, z) e_{r}+u^{\theta}(r, z) e_{\theta}+u^{z}(r, z) e_{z},
$$

where $e_{r}=(\cos \theta, \sin \theta, 0)^{T}, e_{\theta}=(-\sin \theta, \cos \theta, 0)^{T}$, and $e_{z}=(0,0,1)^{T}$ are coordinate axes. The vorticity vector $\omega=\nabla \times u$ has a similar representation,

$$
\begin{gathered}
\omega(r, z)=\omega^{r}(r, z) e_{r}+\omega^{\theta}(r, z) e_{\theta}+\omega^{z}(r, z) e_{z}, \\
\omega^{r}=-u_{z}^{\theta}, \quad \omega^{\theta}=u_{z}^{r}-u_{r}^{z}, \quad \omega^{z}=\frac{1}{r}\left(r u^{\theta}\right)_{r},
\end{gathered}
$$

where for simplicity we have used subscripts to denote partial differentiations. The incompressibility condition $\nabla \cdot u=0$ implies the existence of a stream function

$$
\psi(r, z)=\psi^{r}(r, z) e_{r}+\psi^{\theta}(r, z) e_{\theta}+\psi^{z}(r, z) e_{z},
$$

for which $u=\nabla \times \psi$ and $\omega=-\Delta \psi$. Taking the $\theta$-components of the velocity equation (1.1), the vorticity equation

$$
\omega_{t}+u \cdot \nabla \omega=\omega \cdot \nabla u
$$

and the Poisson equation $-\Delta \psi=\omega$ gives an alternative formulation of the 3D Euler equations

$$
\begin{aligned}
u_{1, t}+u^{r} u_{1, r}+u^{z} u_{1, z} & =2 u_{1} \psi_{1, z}, \\
\omega_{1, t}+u^{r} \omega_{1, r}+u^{z} \omega_{1, z} & =\left(u_{1}^{2}\right)_{z}, \\
-\left[\partial_{r}^{2}+(3 / r) \partial_{r}+\partial_{z}^{2}\right] \psi_{1} & =\omega_{1},
\end{aligned}
$$

\footnotetext{
${ }^{1}$ Indeed, according to the partial regularity result of Caffarelli, Kohn, and Nirenberg [9], any finite-time singularity of the 3D axisymmetric Navier-Stokes equations, if it exists, must lie on the symmetry axis.
} 
where $u_{1}=u^{\theta} / r, \omega_{1}=\omega^{\theta} / r$, and $\psi_{1}=\psi^{\theta} / r$ are transformed angular velocity, vorticity, and stream functions, respectively. ${ }^{2}$ The radial and axial components of the velocity can be recovered from $\psi_{1}$ as

$$
u^{r}=-r \psi_{1, z}, \quad u^{z}=2 \psi_{1}+r \psi_{1, r},
$$

for which the incompressibility condition

$$
\frac{1}{r}\left(r u^{r}\right)_{r}+u_{z}^{z}=0
$$

is satisfied automatically. As shown by [40], $\left(u^{\theta}, \omega^{\theta}, \psi^{\theta}\right)$ must all vanish at $r=0$ if $u$ is a smooth velocity field. Thus $\left(u_{1}, \omega_{1}, \psi_{1}\right)$ are well defined as long as the corresponding solution to (1.1) remains smooth. The reason we choose to work with the transformed variables $\left(u_{1}, \omega_{1}, \psi_{1}\right)$ instead of the original variables $\left(u^{\theta}, \omega^{\theta}, \psi^{\theta}\right)$ is that the equations satisfied by the latter,

$$
\begin{aligned}
u_{t}^{\theta}+u^{r} u_{r}^{\theta}+u^{z} u_{z}^{\theta} & =-\frac{1}{r} u^{r} u^{\theta}, \\
\omega_{t}^{\theta}+u^{r} \omega_{r}^{\theta}+u^{z} \omega_{z}^{\theta} & =\frac{2}{r} u^{\theta} u_{z}^{\theta}+\frac{1}{r} u^{r} \omega^{\theta}, \\
-\left[\Delta-\left(1 / r^{2}\right)\right] \psi^{\theta} & =\omega^{\theta},
\end{aligned}
$$

have a formal singularity at $r=0$, which is inconvenient to work with numerically.

We shall numerically solve the transformed equations (2.1) on the cylinder

$$
D(1, L)=\{(r, z): 0 \leq r \leq 1,0 \leq z \leq L\}
$$

with the initial condition

$$
u_{1}^{0}(r, z)=100 \mathrm{e}^{-30\left(1-r^{2}\right)^{4}} \sin \left(\frac{2 \pi}{L} z\right), \quad \omega_{1}^{0}(r, z)=\psi_{1}^{0}(r, z)=0 .
$$

The solution is subject to a periodic boundary condition in $z$,

$$
u_{1}(r, 0, t)=u_{1}(r, L, t), \quad \omega_{1}(r, 0, t)=\omega_{1}(r, L, t), \quad \psi_{1}(r, 0, t)=\psi_{1}(r, L, t),
$$

and a no-flow boundary condition on the solid boundary $r=1$ :

$$
\psi_{1}(1, z, t)=0 .
$$

The pole condition

$$
u_{1, r}(0, z, t)=\omega_{1, r}(0, z, t)=\psi_{1, r}(0, z, t)=0
$$

is also enforced at the symmetry axis $r=0$ to ensure the smoothness of the solution. The initial condition (2.2a) describes a purely rotating eddy in a periodic cylinder, and it satisfies special odd-even symmetries at the planes $z_{i}=\frac{i}{4} L, i=0,1,2,3$. Specifically, $u_{1}^{0}$ is even at $z_{1}, z_{3}$, it is odd at $z_{0}, z_{2}$, and $\omega_{1}^{0}, \psi_{1}^{0}$ are both odd at all $z_{i}$ 's. These symmetry properties are preserved by the equations (2.1), so instead of solving the problem (2.1)-(2.2) on the entire cylinder $D(1, L)$, it suffices to consider the problem on the quarter cylinder $D\left(1, \frac{1}{4} L\right)$, with the periodic boundary condition $(2.2 \mathrm{~b})$ replaced by appropriate symmetry boundary conditions. It is also interesting to notice that the boundaries of $D\left(1, \frac{1}{4} L\right)$ behave like "impermeable walls":

$$
u^{r}=-r \psi_{1, z}=0 \quad \text { on } \quad r=1, \quad u^{z}=2 \psi_{1}+r \psi_{1, r}=0 \quad \text { on } \quad z=0, \frac{1}{4} L,
$$

which is a consequence of the no-flow boundary condition (2.2c) and the odd symmetry of $\psi_{1}$.

\footnotetext{
${ }^{2}$ These variables should not be confused with the components of the velocity, vorticity, and stream function vectors.
}

Copyright $@$ by SIAM. Unauthorized reproduction of this article is prohibited. 
3. Outline of the numerical method. The potential formation of a finite-time singularity from the initial condition (2.2a) makes the numerical solution of the initialboundary value problem (2.1)-(2.2) a challenging and difficult task. In this section, we describe a special mesh adaptation strategy (section 3.1) and a B-spline based Galerkin Poisson solver (section 3.2), which are essential to the accurate computation of the nearly singular solutions. The overall algorithm is outlined in section 3.3.

3.1. The adaptive (moving) mesh algorithm. Singularities (blowups) are abundant in mathematical models of physical systems. Examples include the semilinear parabolic equations describing the blowup of the temperature of a reacting medium, such as a burning gas [23]; the nonlinear Schrödinger equations describing the self-focusing of electromagnetic beams in a nonlinear medium [42]; and the aggregation equations describing the concentration of interacting particles [36]. Often, singularities occur on increasingly small length and time scales, which necessarily requires some form of mesh adaptation. Further, finite-time singularities usually evolve in a "self-similar" manner when singularity time is approached. An adaptive mesh designed for singularity detection must correctly capture this behavior in the numerical solution.

Several methods have been proposed to compute (self-similar) singularities. In [42], a dynamic rescaling algorithm is used to solve the cubic Schrödinger equation. The main advantage of the method is that the rescaled equation is nonsingular and the rescaled variable is uniformly bounded in appropriate norms. The disadvantage is that the fixed-sized mesh is spread apart by rescaling, so accuracy is inevitably lost far from the singularity.

In [4], a rescaling algorithm is proposed for the numerical solution of the semilinear heat equation, based on the idea of adaptive mesh refinement. The method repeatedly refines the mesh in the "inner" region of the singularity and rescales the inner solution so that it remains uniformly bounded. The main advantage of the method is that it achieves uniform accuracy across the entire computational domain and is applicable to more general problems. The disadvantage is that it requires a priori knowledge of the singularity and is not easily adaptable to elliptic equations (especially in multiple space dimensions) due to the use of irregular mesh.

The moving mesh method [35] provides a very general framework for mesh adaptation and has been applied in various contexts, for example, the semilinear heat equation [7] and the nonlinear Schrödinger equation [6]. The main idea of the method is to construct the mesh based on a certain equidistribution principle, for example, the equipartition of the arc length function. In one dimension this completely determines the mesh, while in higher dimensions additional constraints are needed to specify mesh shapes and orientations. The meshes are automatically evolved with the underlying solution, typically by solving a moving mesh partial differential equation (MMPDE).

While being very general, the "conventional" moving mesh method has the following issues when applied to singularity detection. First, it requires explicit knowledge of the singularity, for example, its scaling exponent, in order to correctly capture the singularity [34]. Second, it tends to place too many mesh points near the singularity while leaving too few elsewhere, which can cause instability. Third, mesh smoothing, an operation necessary for maintaining stability, can significantly limit the maximum resolution power of the mesh. Finally, the moving mesh method computes only a discrete approximation of the mesh mapping function, which can result in catastrophic loss of accuracy in the computation of a singularity (see section 3.3). 
For the particular blowup candidate considered in this paper, preliminary uniform mesh computations suggest that the vorticity function tends to concentrate at a single point. In addition, the solution appears to remain slowly varying and smooth outside a small neighborhood of the singularity. These observations motivate the following special mesh adaptation strategy.

The adaptive mesh covering the computational domain $D\left(1, \frac{1}{4} L\right)$ is constructed from a pair of analytic mesh mapping functions

$$
r=r(\rho), \quad z=z(\eta),
$$

which are defined on $[0,1]$, are infinitely differentiable, and have a density that is even at both 0 and 1 . The even symmetries of the mesh density ensure that the resulting mesh can be extended smoothly to the full cylinder $D(1, L)$. The mesh mapping functions contain a small number of parameters, which are dynamically adjusted so that a certain fraction of the mesh points (e.g., $50 \%$ along each dimension) is placed in a small neighborhood of the singularity. Once the mesh mapping functions are constructed, the computational domain $D\left(1, \frac{1}{4} L\right)$ is covered with a tensor-product mesh:

$$
\mathcal{G}_{0}=\left\{\left(r_{j}, z_{i}\right): 0 \leq i \leq M, 0 \leq j \leq N\right\}
$$

where

$$
r_{j}=r\left(j h_{r}\right), z_{i}=z\left(i h_{z}\right), \quad h_{r}=1 / N, h_{z}=1 / M .
$$

The precise definition and construction of the mesh mapping functions are detailed in Appendix A.

The mesh is evolved using the following procedure. Starting from a reference time $t_{0}$, the "singularity region" $S_{0}$ at $t_{0}$ is identified as the smallest rectangle in the $r z$-plane that encloses the set

$$
D_{\delta_{0}}\left(t_{0}\right):=\left\{(r, z) \in D\left(1, \frac{1}{4} L\right):\left|\omega\left(r, z, t_{0}\right)\right| \geq \delta_{0}\left\|\omega\left(\cdot, t_{0}\right)\right\|_{\infty}\right\}, \quad \delta_{0} \in(0,1) .
$$

Once $S_{0}$ is determined, an adaptive mesh $\mathcal{G}_{0}$ is fit to $S_{0}$ and the solution is advanced in the $\rho \eta$-space by one time step to $t_{1}$. The singularity region $S_{1}$ at $t_{1}$ is then computed and compared with $S_{0}$. If the ratios between the sides of $S_{1}$ and $S_{0}$ (in either dimension) drop below a certain threshold (e.g., $80 \%$ ), which indicates the support of the maximum vorticity has shrunk by a sufficient amount, or if the maximum vorticity at $t_{1}$ is "too close" to the boundaries of $S_{0}$,

$$
\max _{(r, z) \in \partial S_{0}}\left|\omega\left(r, z, t_{1}\right)\right| \geq \delta_{1}\left\|\omega\left(\cdot, t_{1}\right)\right\|_{\infty}, \quad \delta_{1} \in\left(\delta_{0}, 1\right),
$$

which indicates the maximum vorticity is about to leave $S_{0}$, then a new mesh $\mathcal{G}_{1}$ is computed and adapted to $S_{1}$. In the event of a mesh update, the solution is interpolated from $\mathcal{G}_{0}$ to $\mathcal{G}_{1}$ in the $\rho \eta$-space using an 8th-order piecewise polynomial interpolation in $\rho$ and a spectral interpolation in $\eta$. The whole procedure is then repeated with $\mathcal{G}_{0}$ replaced by $\mathcal{G}_{1}$ and $t_{0}$ replaced by $t_{1}$.

We remark that the mesh update criterion (3.1) is designed to prevent the peak vorticity from escaping the singularity region, as is the case in one of our earlier computations where the singularity keeps moving toward the symmetry axis. Since 
in the current computation the singularity is fixed at the corner $\tilde{q}_{0}=(1,0)^{T}$, the criterion (3.1) has practically no effect.

The mesh adaptation strategy described above has several advantages compared with the conventional moving mesh method. First, it can automatically resolve a self-similar singularity regardless of its scalings, provided that the singularity has a bell-shaped similarity profile, which is what we observe in our case (see Figure 1(b)). This is crucial to the success of our computations, because the (axisymmetric) Euler equations allow for infinitely many self-similar scalings (see section 4.7), which means that the scaling exponent of the singularity cannot be determined a priori. Second, the method always places enough mesh points (roughly 50\% along each dimension) outside the singularity region, ensuring a well-behaved and stable mesh (see section 4.1). Third, the explicit control of the mesh mapping functions eliminates the need of mesh smoothing, which allows the mesh to achieve arbitrarily high resolutions. Finally, the analytic representation of the mesh mapping functions ensures accurate approximations of space derivatives, hence greatly improving the quality of the computed solutions (see section 3.3).

3.2. The B-spline based Galerkin Poisson solver. A key observation we made from our computations is that the overall accuracy of the numerical solution of the initial-boundary value problem (2.1)-(2.2) depends crucially on the accuracy of the Poisson solver. Among the methods commonly used for solving Poisson equations, namely finite difference, finite element Galerkin, and finite element collocation, we have chosen the Galerkin method for both its high accuracy and for its rigorous theoretic framework, which makes the error analysis much easier.

We have designed and implemented a B-spline based Galerkin method for the Poisson equation (2.1c). Compared with the "conventional" Galerkin methods based on piecewise polynomials, the B-spline based method requires no mesh generation and hence is much easier to implement. More importantly, the method can achieve arbitrary global smoothness and approximation order with relative ease and few degrees of freedom, in contrast to the conventional piecewise polynomial based methods. This makes the method a natural choice for our problem.

The Poisson equation (2.1c) is solved in the $\rho \eta$-space using the following procedure. First, the equation is recast in the $\rho \eta$-coordinates:

$$
-\frac{1}{r^{3} r_{\rho}}\left(r^{3} \frac{\psi_{\rho}}{r_{\rho}}\right)_{\rho}-\frac{1}{z_{\eta}}\left(\frac{\psi_{\eta}}{z_{\eta}}\right)_{\eta}=\omega, \quad(\rho, \eta) \in[0,1]^{2},
$$

where for clarity we have written $\psi$ for $\psi_{1}$ and $\omega$ for $\omega_{1}$. Next, the equation is multiplied by $r^{3} r_{\rho} z_{\eta} \phi$ and is integrated over the domain $[0,1]^{2}$ where $\phi \in V$ (to be defined below) is a suitable test function. After a routine integration by parts, this yields the desired weak formulation of (2.1c), which reads as follows: find $\psi \in V$ such that

$$
\begin{aligned}
a(\psi, \phi) & :=\int_{[0,1]^{2}}\left(\frac{\psi_{\rho}}{r_{\rho}} \frac{\phi_{\rho}}{r_{\rho}}+\frac{\psi_{\eta}}{z_{\eta}} \frac{\phi_{\eta}}{z_{\eta}}\right) r^{3} r_{\rho} z_{\eta} d \rho d \eta \\
& =\int_{[0,1]^{2}} \omega \phi r^{3} r_{\rho} z_{\eta} d \rho d \eta=: f(\phi) \quad \forall \phi \in V,
\end{aligned}
$$

where (recall the odd symmetry of $\psi$ at $\eta=0,1$ )

$$
\begin{aligned}
V=\operatorname{span}\left\{\phi \in H^{1}[0,1]^{2}: \phi(-\rho, \eta)\right. & =\phi(\rho, \eta), \\
\phi(1, \eta) & =0, \phi(\rho, \ell-\eta)=-\phi(\rho, \ell+\eta) \forall \ell \in \mathbb{Z}\} .
\end{aligned}
$$

Copyright (c) by SIAM. Unauthorized reproduction of this article is prohibited. 
To introduce Galerkin approximation, we define the finite-dimensional subspace of weighted uniform B-splines [30] of even order $k$ :

$$
V_{h}:=V_{w, h}^{k}=\operatorname{span}\left\{w(\rho) b_{j, h_{r}}^{k}(\rho) b_{i, h_{z}}^{k}(\eta)\right\} \cap V,
$$

where $w(\rho)$ is a nonnegative weight function of order 1 vanishing on $\rho=1$,

$$
w(\rho) \sim(1-\rho), \quad \rho \rightarrow 1^{-},
$$

and $b_{\ell, h}^{k}(s)=b^{k}((s / h)-(\ell-k / 2))$ are shifted and rescaled uniform B-splines of order $k$. The Galerkin formulation then reads as follows: find $\psi_{h} \in V_{h}$ such that

$$
a\left(\psi_{h}, \phi_{h}\right)=f\left(\phi_{h}\right) \quad \forall \phi_{h} \in V_{h} .
$$

With suitably chosen basis functions of $V_{h}$, this gives rise to a symmetric, positive definite linear system $A x=b$ which can be solved to yield the Galerkin solution $\psi_{h}$. The detailed construction of the linear system is given in Appendix B.

The parameters used in our computations are $k=6$ and $w(\rho)=1-\rho^{2}$.

Using the theory of quasi interpolants, it can be shown that

$$
\int_{[0,1]^{2}}\left|\nabla \psi-\nabla \psi_{h}\right|^{2} r^{3} d r d z \leq C_{0} C_{r z}\left(h_{r} h_{z}\right)^{k-1} \int_{[0,1]^{2}} \sum_{|\alpha| \leq k-1}\left|\tilde{\partial}^{\alpha} \nabla \psi\right|^{2} r^{3} d r d z
$$

where $\nabla=\left(\partial_{r}, \partial_{z}\right)^{T}, \tilde{\partial}^{\alpha}=\partial_{\rho}^{\alpha_{1}} \partial_{\eta}^{\alpha_{2}}$ are differential operators in $r z$ - and $\rho \eta$-planes, respectively, $C_{r z}$ is a mesh-mapping dependent constant, and $C_{0}$ is an absolute constant. In our computations, the constant $C_{r z}$ is observed to be very close to 1 for all times, which confirms the stability of the Galerkin solver.

The detailed error analysis of the Poisson solver will be reported in a separate paper.

3.3. The overall algorithm. Given an adaptive mesh $\mathcal{G}_{0}$ and the data $\left(u_{1}, \omega_{1}\right)$ defined on $\mathcal{G}_{0}$, the solution is advanced using the following procedure. First, the Poisson equation (2.1c) is solved for $\psi_{1}$ in the $\rho \eta$-space using a 6 th-order B-spline based Galerkin method (section 3.2). Second, the 2D velocity $\tilde{u}=\left(u^{r}, u^{z}\right)^{T}$ is evaluated at the grid points using $(2.1 \mathrm{~d})$. Third, an adaptive time step $\delta_{t}$ is computed on $\mathcal{G}_{0}$ so that the CFL condition is satisfied with a suitably small CFL number $\nu$ (e.g., 0.5), and the relative growth of the solution in one step does not exceed a small threshold $\epsilon_{t}$ (e.g., $\left.5 \%\right)$. Finally, the solution $\left(u_{1}, \omega_{1}\right)$ is advanced according to $(2.1 \mathrm{a})-(2.1 \mathrm{~b})$ by $\delta_{t}$ using an explicit 4th-order Runge-Kutta method, and the mesh $\mathcal{G}_{0}$ is adapted to the new solution if necessary (section 3.1).

In the last step of the algorithm, the evolution equations for $u_{1}$ and $\omega_{1}$ are semidiscretized in the $\rho \eta$-space, where the space derivatives are expressed in the $\rho \eta$-coordinates and are approximated using 6th-order centered difference formulas, e.g.,

$$
v_{r}\left(r_{j}, z_{i}\right)=:\left(v_{r}\right)_{i j}=\frac{\left(v_{\rho}\right)_{i j}}{\left(r_{\rho}\right)_{j}} \approx \frac{1}{\left(r_{\rho}\right)_{j}}\left(Q_{\rho, 6} v_{i, \cdot}\right)_{j}, \quad v=u_{1} \quad \text { or } \quad \omega_{1} .
$$

Here, as usual,

$$
Q_{\rho, 6}:=D_{\rho, 0}\left(I-\frac{1}{6} h_{r}^{2} D_{\rho,+} D_{\rho,-}+\frac{1}{30} h_{r}^{4} D_{\rho,+}^{2} D_{\rho,-}^{2}\right)
$$


denotes the standard 6th-order centered approximation to $\partial_{\rho}$, and

$$
\left(D_{\rho, \pm} v_{i,}\right)_{j}:= \pm \frac{1}{h_{r}}\left(v_{i, j \pm 1}-v_{i, j}\right), \quad\left(D_{\rho, 0} v_{i, \cdot}\right)_{j}:=\frac{1}{2 h_{r}}\left(v_{i, j+1}-v_{i, j-1}\right)
$$

denote the standard forward, backward, and centered difference operators, respectively. Note that the derivative $r_{\rho}$ of the mesh mapping function is computed directly from the analytic representation of $r$ without any difference approximation. This is crucial for the accurate evaluation of $v_{r}$, especially in "singularity regions" where the inverse mesh density $r_{\rho}$ is close to 0 and is nearly constant (Appendix A; in particular, see (A.3)). When $r_{\rho}$ is small and nearly constant, a high-order difference approximation of $r_{\rho}$ tends to be contaminated by catastrophic cancellation, and the discretely approximated values of $r_{\rho}$ can have large relative errors or even become negative, causing failures of the entire computation. By computing $r_{\rho}$ directly from the analytic representation of $r$, this problem is avoided and the solution is ensured to be accurately approximated even in regions where the singularity is about to form and where $r_{\rho} \approx c \ll 1$. This also explains why the conventional moving mesh method is not suitable for singularity computations where high accuracy is demanded, because the method computes only a discrete approximation of the mesh mapping function, which necessarily requires a difference approximation of $r_{\rho}$ in the evaluation of a space derivative $v_{r}$. Without mesh smoothing, this can cause instability, while with mesh smoothing the mesh resolution will be inevitably limited, which is undesired.

The centered difference formulas described above need to be supplemented by numerical boundary conditions near $\rho, \eta=0,1$. Along the $\eta$-dimension, the symmetry condition

$$
v_{-i, j}=-v_{i, j}, \quad v_{M+i, j}= \pm v_{M-i, j}, \quad 1 \leq i \leq 3,0 \leq j \leq N,
$$

is used near $\eta=0$ and $\eta=1$, where the + sign applies to $u_{1}$ and the - sign applies to $\omega_{1}$. Along the $\rho$-dimension, the symmetry condition

$$
v_{i,-j}=v_{i, j}, \quad 0 \leq i \leq M, 1 \leq j \leq 3,
$$

is used near the axis $\rho=0$ and the extrapolation condition

$$
\left(D_{\rho,-}^{7} v_{i, \cdot}\right)_{N+j}=0, \quad 0 \leq i \leq M, 1 \leq j \leq 3,
$$

is applied near the solid boundary $\rho=1 .^{3}$ The extrapolation condition is known to be GKS stable for linear hyperbolic problems [28, Theorem 13.1.3], and it is expected to remain stable when applied to the Euler equations as long as the underlying solution is sufficiently smooth.

Using the superconvergence properties of the Poisson solver at the grid points (to be proved elsewhere), it can be shown that the overall algorithm is formally 6 th-order accurate in space and 4th-order accurate in time. The details of this error analysis will be reported in a separate paper.

4. Numerical results. We have numerically solved the initial-boundary value problem (2.1)-(2.2) on the quarter cylinder $D\left(1, \frac{1}{24}\right)$ (with $L=\frac{1}{6}$ ). The results suggest that the solution develops a singularity in finite time, and we shall provide,

\footnotetext{
${ }^{3}$ While a 6th-order extrapolation condition $\left(D_{\rho,-}^{6} v_{i, \cdot}\right)_{N+j}=0$ suffices to maintain a formal 6thorder accuracy for the overall scheme, we choose the higher-order extrapolation condition for better accuracy.
} 
TABLE 1

Stopping time $t_{e}$ and cause of termination, where $\left(\delta_{r}, \delta_{z}\right)$ denote the minimum mesh spacing in $r$ and $z$, respectively.

\begin{tabular}{ccc}
\hline Mesh size & $t_{e}$ & Cause of termination \\
\hline $1024 \times 1024$ & 0.0035055667206 & $\delta_{r}<\epsilon_{r}$ and $\delta_{z}<\epsilon_{z}$ \\
$1280 \times 1280$ & 0.0035055581996 & $\delta_{z}<\epsilon_{z}$ \\
$1536 \times 1536$ & 0.0035055522856 & $\delta_{z}<\epsilon_{z}$ \\
$1792 \times 1792$ & 0.0035055523092 & $\delta_{r}<\epsilon_{r}$ and $\delta_{z}<\epsilon_{z}$ \\
$2048 \times 2048$ & 0.0035055472037 & $\delta_{r}<\epsilon_{r}$ and $\delta_{z}<\epsilon_{z}$ \\
\hline
\end{tabular}

in what follows, ample evidence to support this finding. We start with an overview of our computations in sections 4.1-4.2, where the effectiveness of the adaptive mesh is demonstrated and the first sign of a finite-time singularity is given. After a careful resolution study of the computed solutions in section 4.3, we proceed to sections $4.4-$ 4.5 , where the asymptotic scalings of the vorticity moments are analyzed in great detail. The results indicate the divergence of the time integral of the maximum vorticity and hence the blowup of the computed solutions. This conclusion is further supported in section 4.6, where the geometric structures of the vorticity direction field are analyzed and the consistency between the blowing-up solutions and the various geometric non-blowup criteria is demonstrated. Once the existence of a finite-time singularity is confirmed, we move on to section 4.7 , where the locally self-similar structure of the blowing-up solutions is examined. The discussion is concluded in section 4.8 with a physical interpretation of the finite-time singularity, where the driving mechanism behind the blowup is investigated.

4.1. Effectiveness of the adaptive mesh. We have numerically solved the problem (2.1)-(2.2) on meshes of size $256 k \times 256 k$ where $k=4,5,6,7,8$. In each computation, the solution is initialized on a uniform mesh, which is then adjusted to the initial data using the adaptive mesh algorithm described in section 3.1. Once an "optimal" mesh is obtained, the solution is advanced indefinitely in time using the method described in section 3, until either the time step drops below $10^{-12}$, or the minimum mesh spacing drops below $\epsilon_{r}=10^{-15}$ (in $r$ ) or $\epsilon_{z}=10^{-15}\left(\frac{1}{4} L\right.$ ) (in $z$ ), whichever happens first.

Table 1 shows the stopping time $t_{e}$ and the cause of termination for each resolution run. For all the computations shown in the table, the total number of remeshing steps is about 130, and this number depends on the choice of the parameters $\delta_{0}, \delta_{1}$ (see (3.1)) but is relatively insensitive to the particular choice of the mesh mapping functions. As indicated by the mostly decreasing stopping time (with respect to the increasing resolution) and the vanishing minimum mesh spacings, the solution seems to develop a very singular structure in finite time. To determine the nature of the singular structure and to see how well the adaptive mesh resolves it, we plot in Figure 1 the vorticity function $|\omega|$ computed on the $1024 \times 1024$ mesh at $t=0.003505$, in both the $r z$-coordinates (Figure 1(a)) and the $\rho \eta$-coordinates (Figure 1(b)). The $r z$ plot suggests that the singular structure could be a point-singularity at the corner $\tilde{q}_{0}=(1,0)^{T}$, which corresponds to a ring-singularity on the solid boundary due to the axial symmetry. The $\rho \eta$-plot, on the other hand, shows that a good portion of the mesh points (roughly 50\% along each dimension) are consistently placed in regions where $|\omega|$ is comparable with the maximum vorticity $\|\omega\|_{\infty}$, hence demonstrating the effectiveness of the adaptive mesh. To quantitatively measure the maximum resolution 


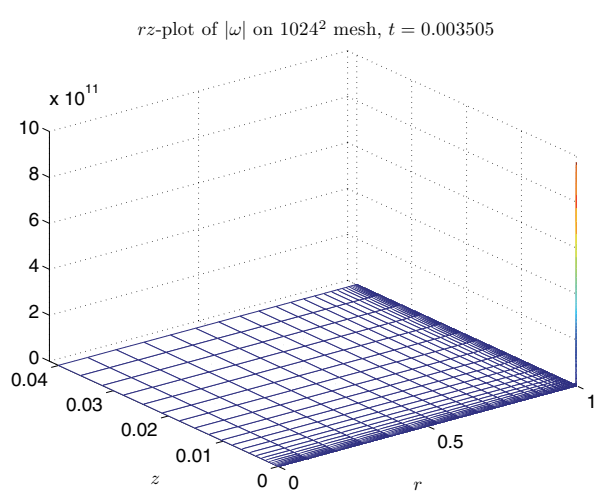

(a) $r z$-plane

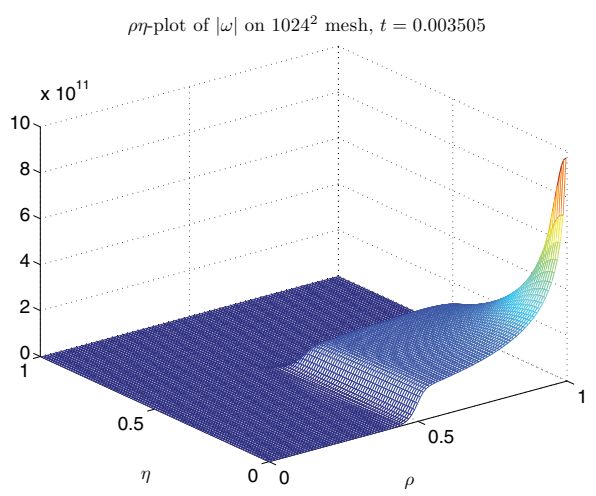

(b) $\rho \eta$-plane

FIG. 1. The vorticity function $|\omega|$ on the $1024 \times 1024$ mesh at $t=0.003505$, in (a) rz-coordinates and (b) $\rho \eta$-coordinates, where for clarity only one-tenth of the mesh lines are displayed along each dimension.

TABLE 2

Mesh compression ratios $\left(p_{\infty}, q_{\infty}\right)$ and effective mesh resolutions $\left(M_{\infty}, N_{\infty}\right)$ at the location of the maximum vorticity at $t=0.003505$.

\begin{tabular}{ccccc}
\hline \multirow{2}{*}{ Mesh size } & \multicolumn{4}{c}{$t=0.003505$} \\
\cline { 2 - 5 } & $p_{\infty}$ & $M_{\infty}$ & $q_{\infty}$ & $N_{\infty}$ \\
\hline $1024 \times 1024$ & $1.9456 \times 10^{9}$ & $1.9923 \times 10^{12}$ & $1.6316 \times 10^{9}$ & $1.6708 \times 10^{12}$ \\
$1280 \times 1280$ & $1.9530 \times 10^{9}$ & $2.4999 \times 10^{12}$ & $1.6285 \times 10^{9}$ & $2.0844 \times 10^{12}$ \\
$1536 \times 1536$ & $1.9444 \times 10^{9}$ & $2.9866 \times 10^{12}$ & $1.6328 \times 10^{9}$ & $2.5079 \times 10^{12}$ \\
$1792 \times 1792$ & $1.9504 \times 10^{9}$ & $3.4951 \times 10^{12}$ & $1.6344 \times 10^{9}$ & $2.9288 \times 10^{12}$ \\
$2048 \times 2048$ & $1.9503 \times 10^{9}$ & $3.9942 \times 10^{12}$ & $1.6330 \times 10^{9}$ & $3.3444 \times 10^{12}$ \\
\hline
\end{tabular}

power achieved by the adaptive mesh, we define the mesh compression ratios

$$
p_{\infty}:=\frac{L}{4 z^{\prime}\left(\eta_{\infty}\right)}, \quad q_{\infty}:=\frac{1}{r^{\prime}\left(\rho_{\infty}\right)}
$$

and the effective mesh resolutions

$$
M_{\infty}:=p_{\infty} M=\frac{L M}{4 z^{\prime}\left(\eta_{\infty}\right)}, \quad N_{\infty}:=q_{\infty} N=\frac{N}{r^{\prime}\left(\rho_{\infty}\right)}
$$

at the location $\left(\rho_{\infty}, \eta_{\infty}\right)^{T} \equiv(1,0)^{T}$ of the maximum vorticity $\|\omega\|_{\infty}$. The values of these quantities computed at $t=0.003505$ are summarized in Table 2 .

The above analysis confirms the effectiveness of the adaptive mesh in the "inner region" where the vorticity function $|\omega|$ is most singular, but it says nothing about the quality of the mesh outside the inner region. To address this issue, we plot in Figure 2(a) the trajectories of the $r$-mesh points

$$
\rho_{j}^{*}:=\rho_{\infty}-\frac{j}{10} \equiv 1-\frac{j}{10}, \quad j=1, \ldots, 9,
$$

which can be viewed as "Lagrangian markers" equally spaced (in $\rho$ ) away from the location of the maximum vorticity $\rho_{\infty} \equiv 1$. The ordinate of the figure represents the distance between the selected mesh points and the location of the maximum vorticity,

$$
d_{r, j}^{*}:=\frac{1}{1}\left[r\left(\rho_{\infty}\right)-r\left(\rho_{j}^{*}\right)\right] \equiv 1-r\left(\rho_{j}^{*}\right), \quad j=1, \ldots, 9,
$$




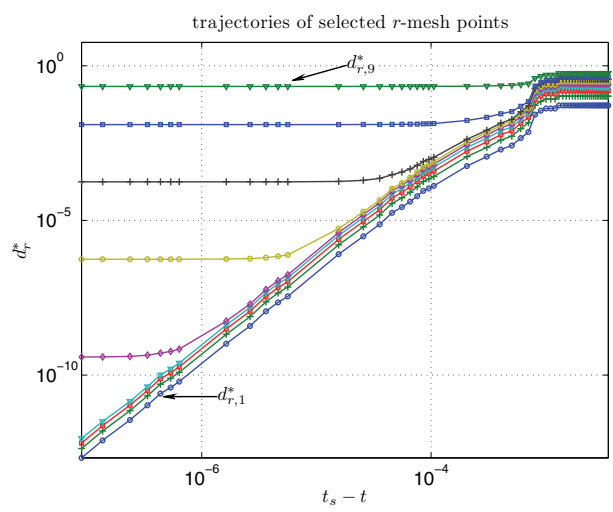

(a) full view

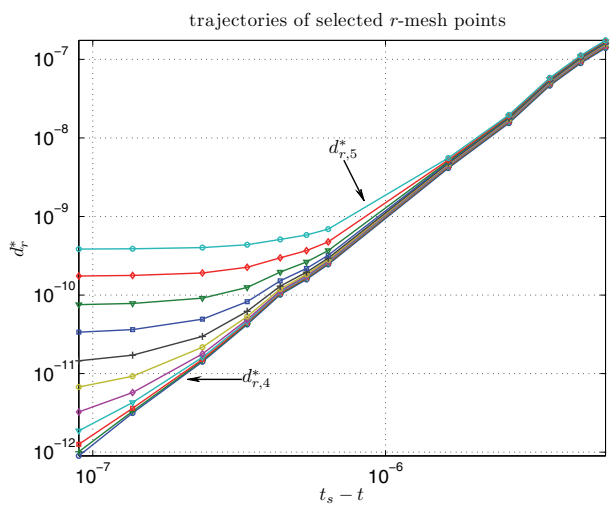

(b) detailed view

FIG. 2. Trajectories of selected $r$-mesh points on the $2048 \times 2048$ mesh in log-log scale (see text for explanation). The last time instant shown in the figure is $t_{e}$, the stopping time.

expressed as a fraction of the total length of the computational domain ( 1 in this case). The abscissa of the figure represents $t_{s}-t$ where $t_{s}$ is the predicted singularity time (see section 4.4). As is clear from the figure, the $40 \%$ mesh points that lie closest to $\rho_{\infty}$ are always placed in the inner region while the $50 \%$ points farthest away from $\rho_{\infty}$ eventually move into the outer region. The $10 \%$ points lying between the inner and outer regions belong to the "transition region" and are shown in greater detail in Figure 2(b). A similar analysis performed along the $z$-dimension shows that the $z$-adaptive mesh has a completely similar character.

To see how well the solution is resolved in the transition region, we define

$$
\Omega_{j}^{*}:=\frac{1}{\|\omega\|_{\infty}} \sup _{(r, z) \in D_{j}^{*}}|\omega(r, z)|, \quad j=1, \ldots, 9,
$$

where

$$
D_{j}^{*}=D\left(1, \frac{1}{4} L\right) \backslash\left[r\left(\rho_{j}^{*}\right), 1\right] \times\left[0, z\left(\eta_{j}^{*}\right)\right], \quad \eta_{j}^{*}:=\eta_{\infty}+\frac{j}{10} \equiv \frac{j}{10},
$$

is the portion of the quarter cylinder $D\left(1, \frac{1}{4} L\right)$ outside the region $\left[r\left(\rho_{j}^{*}\right), 1\right] \times\left[0, z\left(\eta_{j}^{*}\right)\right]$. As is clear from Figure 3(a), the values of $\Omega_{j}^{*}$ stay nearly constant for $j \leq 4$ and steadily decay for $j \geq 5$, consistent with the observation that the $40 \%$ points lying closest to $\left(\rho_{\infty}, \eta_{\infty}\right)$ belong to the inner region while the $50 \%$ points farthest away from $\left(\rho_{\infty}, \eta_{\infty}\right)$ belong to the outer region. Within the transition region to which the remaining $10 \%$ points belong, the vorticity function $|\omega|$ varies smoothly from $10^{-3}\|\omega\|_{\infty}$ to $10^{-1}\|\omega\|_{\infty}$ (Figure 3(b)). This suggests that the adaptive mesh generates a nearly uniform representation of the computed solution across the entire computational domain, hence confirming its efficacy.

To analyze the performance of the Poisson solver, in particular that of the linear solve $A x=b$, we define as in [1] the componentwise backward errors of the first and second kinds,

$$
\omega_{i}=\max _{j} \frac{\left|A^{(i)} \hat{x}-b^{(i)}\right|_{j}}{\left\{\left|A^{(i)}\right||\hat{x}|+f^{(i)}\right\}_{j}}, \quad i=1,2
$$




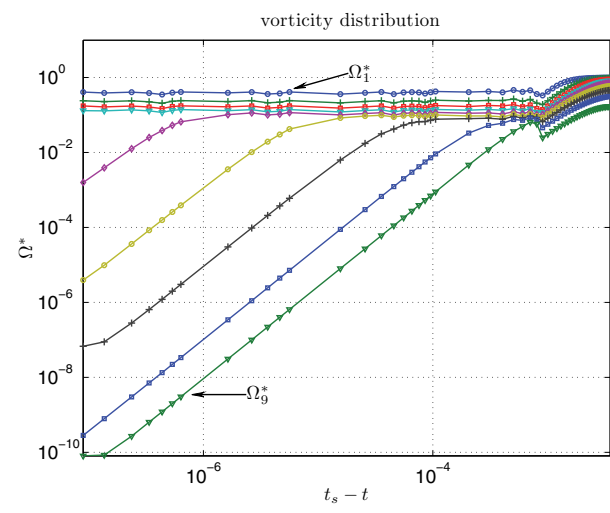

(a) full view

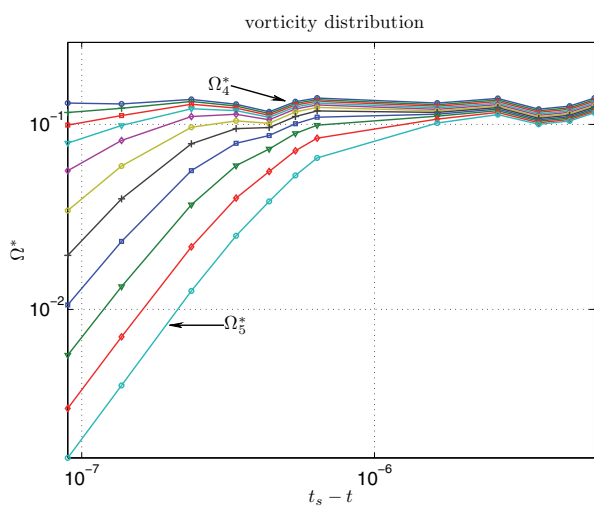

(b) detailed view

FIG. 3. Vorticity distribution on the $2048 \times 2048$ mesh in log-log scale (see text for explanation). The last time instant shown in the figure is $t_{e}$, the stopping time.

TABLE 3

Backward errors of the linear solve $A x=b$ associated with (3.2b) at $t=0.003505$.

\begin{tabular}{cccccc}
\hline \multirow{2}{*}{ Mesh size } & $\omega_{1}$ & $\kappa_{\omega_{1}}$ & $\omega_{2}$ & $\kappa_{\omega_{2}}$ & $\|\delta x\|_{\infty} /\|x\|_{\infty}$ \\
\cline { 2 - 6 } & $4.2456 \times 10^{-12}$ & 974.28 & $5.0563 \times 10^{-20}$ & $1.6772 \times 10^{7}$ & $4.1372 \times 10^{-9}$ \\
\hline $256 \times 256$ & $5.8812 \times 10^{-15}$ & 1247.29 & $1.8902 \times 10^{-23}$ & $2.3027 \times 10^{7}$ & $7.3360 \times 10^{-12}$ \\
$512 \times 512$ & $1.0843 \times 10^{-15}$ & 1788.84 & $2.1290 \times 10^{-23}$ & $5.2033 \times 10^{7}$ & $1.9407 \times 10^{-12}$ \\
$768 \times 768$ & $1.4721 \times 10^{-15}$ & 6748.83 & $6.4433 \times 10^{-23}$ & $9.2646 \times 10^{7}$ & $9.9408 \times 10^{-12}$ \\
$1024 \times 1024^{\dagger}$ & 1.472003505 \\
\hline+ For technil
\end{tabular}

and the componentwise condition numbers of the first and second kinds $\kappa_{\omega_{1}}, \kappa_{\omega_{2}}$. Here $\hat{x}$ is the numerical approximation to the exact solution $x$ and

$$
f^{(1)}=\left|b^{(1)}\right|, \quad f^{(2)}=\left|A^{(2)}\right| e\|\hat{x}\|_{\infty}, \quad e \text { is the vector of all ones. }
$$

The equations in the linear system are classified as follows: let $w=|A||\hat{x}|+|b|$ be the vector of denominators in the definition of $\omega_{i}$. If $w_{j}>\tau_{j}$ for a user-defined threshold $\tau_{j}$, then the $j$ th equation is said to belong to the first category $(i=1)$; otherwise it is said to belong to the second category $(i=2)$. To leading-order approximation, the error $\delta x=\hat{x}-x$ of the linear solve satisfies [1]

$$
\frac{\|\delta x\|_{\infty}}{\|x\|_{\infty}} \leq \omega_{1} \kappa_{\omega_{1}}+\omega_{2} \kappa_{\omega_{2}} .
$$

Compared with the standard norm based error metrics, the error predicted by (4.1) tends to give a much tighter bound for the actual error, especially when $A$ is badly row-scaled [1].

Table 3 shows the backward errors (4.1) as well as other related error metrics computed for the linear system associated with the Poisson solve (3.2b). It can be observed that both condition numbers $\kappa_{\omega_{1}}, \kappa_{\omega_{2}}$ grow roughly like $h^{-2}$ where $h:=$ $\min \left\{h_{r}, h_{z}\right\}=\min \{1 / M, 1 / N\}$ is the (uniform) mesh spacing in the $\rho \eta$-space. It can also be observed that the value of $\kappa_{\omega_{2}}$ is considerably larger than that of $\kappa_{\omega_{1}}$, but the backward error $\omega_{2}$ is so small that the net contribution of $\omega_{2} \kappa_{\omega_{2}}$ is negligible 
TABLE 4

Forward errors of the linear solve $A x=b$ and of the discrete problem $(3.2 \mathrm{~b})$ at $t=0.003505$.

\begin{tabular}{ccccc}
\hline \multirow{2}{*}{ Mesh size } & $\hat{4}$ Sup-norm relative error at $t=0.003505$ \\
\cline { 2 - 5 } & $\hat{x}$ & $\hat{\psi}_{h}$ & $\hat{\psi}_{h, r}$ & $\hat{\psi}_{h, z}$ \\
\hline $1024 \times 1024$ & $3.9638 \times 10^{-14}$ & $1.6697 \times 10^{-14}$ & $4.2104 \times 10^{-12}$ & $5.3310 \times 10^{-12}$ \\
$1280 \times 1280$ & $4.1397 \times 10^{-14}$ & $1.1431 \times 10^{-14}$ & $5.6280 \times 10^{-12}$ & $6.4547 \times 10^{-12}$ \\
$1536 \times 1536$ & $7.0504 \times 10^{-14}$ & $4.8934 \times 10^{-14}$ & $1.1191 \times 10^{-11}$ & $9.3045 \times 10^{-12}$ \\
$1792 \times 1792$ & $4.3910 \times 10^{-14}$ & $1.1045 \times 10^{-14}$ & $9.4986 \times 10^{-12}$ & $1.4097 \times 10^{-11}$ \\
$2048 \times 2048$ & $6.9127 \times 10^{-14}$ & $3.3393 \times 10^{-14}$ & $1.2582 \times 10^{-11}$ & $1.4449 \times 10^{-11}$ \\
\hline
\end{tabular}

TABLE 5

Time step $\delta_{t}$ at selected time $t$.

\begin{tabular}{cccccc}
\hline \multirow{2}{*}{ Mesh size } & \multicolumn{5}{c}{$\delta_{t}$} \\
\cline { 2 - 6 } & $t=0^{\dagger}$ & $t=0.003$ & $t=0.0034$ & $t=0.0035$ & $t=0.003505$ \\
\hline $1024 \times 1024$ & $1 \times 10^{-6}$ & $2.8754 \times 10^{-7}$ & $4.9502 \times 10^{-8}$ & $2.8831 \times 10^{-9}$ & $2.4240 \times 10^{-10}$ \\
$1280 \times 1280$ & $1 \times 10^{-6}$ & $2.3120 \times 10^{-7}$ & $3.9636 \times 10^{-8}$ & $2.2983 \times 10^{-9}$ & $2.5772 \times 10^{-10}$ \\
$1536 \times 1536$ & $1 \times 10^{-6}$ & $1.9165 \times 10^{-7}$ & $3.2907 \times 10^{-8}$ & $1.9165 \times 10^{-9}$ & $2.2223 \times 10^{-10}$ \\
$1792 \times 1792$ & $1 \times 10^{-6}$ & $1.6578 \times 10^{-7}$ & $2.8451 \times 10^{-8}$ & $1.6418 \times 10^{-9}$ & $1.9122 \times 10^{-10}$ \\
$2048 \times 2048$ & $1 \times 10^{-6}$ & $1.4509 \times 10^{-7}$ & $2.4046 \times 10^{-8}$ & $1.4367 \times 10^{-9}$ & $2.0272 \times 10^{-10}$ \\
\hline$\dagger:$ The maximum time step allowed in our computations is $10^{-6}$.
\end{tabular}

compared with that of $\omega_{1} \kappa_{\omega_{1}}$. As a result, the backward error bound $\|\delta x\|_{\infty} /\|x\|_{\infty}$ of the computed solution remains uniformly small on all meshes.

The backward error analysis as described above is applied only to meshes of size no larger than $1024 \times 1024$, due to a technical restriction of the linear solve package that we use. To complete the picture, we also carry out a forward error analysis where the error of the linear solve $A x=b$ as well as that of the discrete problem $(3.2 \mathrm{~b})$ are estimated directly using a three-step procedure. First, the approximate solution $\hat{x}$ of the linear system is taken as the exact solution and a new right-hand side $\hat{b}=A \hat{x}$ is computed from $\hat{x}$ using 128-bit (quadruple-precision) arithmetic. ${ }^{4}$ Second, the linear system $A x=\hat{b}$ with the new right-hand side $\hat{b}$ is solved numerically, yielding an approximate solution $\tilde{x}$. Finally, the reference and the approximate stream functions $\hat{\psi}_{h}, \tilde{\psi}_{h}$ are assembled from the solution vectors $\hat{x}, \tilde{x}$, and the relative errors of $\hat{x}$ as well as those of $\hat{\psi}_{h}, \hat{\psi}_{h, r}, \hat{\psi}_{h, z}$ are computed. The results of this error analysis are summarized in Table 4. As is clear from the table, the Poisson solver is numerically stable despite the very high compression ratios achieved by the adaptive mesh (Table 2). Combined with the discretization error estimate (3.3), this establishes the convergence of the Poisson solver under mesh refinement.

4.2. First sign of singularity. To examine more closely the nature of the singular structure observed in Figure 1, we report in Tables 5-6 the (variable) time steps $\delta_{t}$ and the maximum vorticity $\|\omega\|_{\infty}$ recorded at selected time instants $t$. We also plot in Figure 4 the double logarithm of the maximum vorticity, $\log \left(\log \|\omega\|_{\infty}\right)$, computed on the coarsest $1024 \times 1024$ and the finest $2048 \times 2048$ meshes. It can be observed from these results that, for each computation, there exists a short time interval right before the stopping time $t_{e}$ in which the solution grows tremendously. This can be readily inferred from the sharp decrease in the time step $\delta_{t}$ (Table 5) as

\footnotetext{
${ }^{4}$ This is implemented using GNU's GMP library.
} 
TABLE 6

Maximum vorticity $\|\omega\|_{\infty}$ at selected time $t$.

\begin{tabular}{cccccc}
\hline \multirow{2}{*}{ Mesh size } & \multicolumn{5}{c}{$\|\omega\|_{\infty}$} \\
\cline { 2 - 6 } & $t=0$ & $t=0.003$ & $t=0.0034$ & $t=0.0035$ & $t=0.003505$ \\
\hline $1024 \times 1024$ & $3.7699 \times 10^{3}$ & $9.0847 \times 10^{4}$ & $4.3127 \times 10^{6}$ & $5.8438 \times 10^{9}$ & $1.2416 \times 10^{12}$ \\
$1280 \times 1280$ & $3.7699 \times 10^{3}$ & $9.0847 \times 10^{4}$ & $4.3127 \times 10^{6}$ & $5.8423 \times 10^{9}$ & $1.2407 \times 10^{12}$ \\
$1536 \times 1536$ & $3.7699 \times 10^{3}$ & $9.0847 \times 10^{4}$ & $4.3127 \times 10^{6}$ & $5.8417 \times 10^{9}$ & $1.2403 \times 10^{12}$ \\
$1792 \times 1792$ & $3.7699 \times 10^{3}$ & $9.0847 \times 10^{4}$ & $4.3127 \times 10^{6}$ & $5.8415 \times 10^{9}$ & $1.2401 \times 10^{12}$ \\
$2048 \times 2048$ & $3.7699 \times 10^{3}$ & $9.0847 \times 10^{4}$ & $4.3127 \times 10^{6}$ & $5.8413 \times 10^{9}$ & $1.2401 \times 10^{12}$ \\
\hline
\end{tabular}

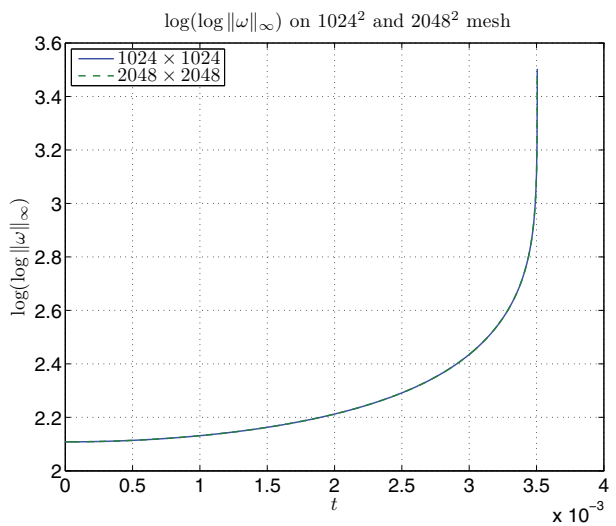

FIG. 4. The double logarithm of the maximum vorticity $\log \left(\log \|\omega\|_{\infty}\right)$ computed on the $1024 \times$ 1024 and the $2048 \times 2048$ meshes. The two curves overlap and are virtually indistinguishable from each other (see section 4.3 below for a detailed resolution study on the nearly singular solutions).

well as the super-double-exponential growth of the maximum vorticity $\|\omega\|_{\infty}$ (Table 6 , Figure 4). In addition, the nearly singular solution seems to converge under mesh refinement (Table 6). These behaviors are characteristic of a blowing-up solution and may be viewed as the first sign of a looming finite-time singularity.

4.3. Resolution study. Of course, neither a rapidly decreasing time step nor a fast growing vorticity can be used alone as evidence for a finite-time singularity. To investigate the issue of finite-time blowup more closely, a much more thorough analysis is needed which, in the first place, requires a careful examination of the accuracy of the computed solutions.

There are several well-established, "standard" methods in the literature to gauge the quality of an Euler computation:

(i) Energy conservation. It is well known that, under suitable regularity assumptions, the solutions of the Euler equations conserve the kinetic energy

$$
E=\frac{1}{2} \int_{D(1, L)}|u|^{2} d x=\frac{1}{2} \int_{0}^{1} \int_{0}^{L}\left(\left|u_{1}\right|^{2}+\left|\psi_{1, r}\right|^{2}+\left|\psi_{1, z}\right|^{2}\right) r^{3} d r d z .
$$

Thus a widely used "quality indicator" for Euler computations is the relative change of the energy integral $E$ over time.

(ii) Enstrophy and enstrophy production rate. Another widely accepted "error 
indicator" for Euler computations is the enstrophy integral

$$
\mathcal{E}=\int_{D(1, L)}|\nabla u|^{2} d x=\int_{D(1, L)}|\omega|^{2} d x
$$

and the enstrophy production rate integral

$$
\mathcal{E}_{p}:=\frac{d \mathcal{E}}{d t}=2 \int_{D(1, L)} \omega \cdot S \omega d x, \quad S=\frac{1}{2}\left(\nabla u+\nabla u^{T}\right) .
$$

These quantities are not conserved over time, but their convergence under mesh refinement provides partial evidence on the convergence of the underlying numerical solutions.

(iii) Energy spectra. For problems defined on periodic domains, it is also a common practice to perform convergence analysis on the energy spectra of the periodic velocity field $u$,

$$
E_{p}(k)=\sum_{|\ell| \in(k-1 / 2, k+1 / 2]}\left|\hat{u}_{\ell}\right|^{2},
$$

and use the results as a measure of the quality of the underlying solutions. Here, as usual, $\hat{u}_{\ell}$ denotes the vector Fourier coefficients of the velocity $u$, which on an $L_{1} \times L_{2} \times L_{3}$ periodic box $B$ is defined by

$$
\begin{aligned}
\hat{u}_{\ell} & =\frac{1}{|B|} \int_{B} u \mathrm{e}^{-\mathrm{i} \ell \cdot x} d x \\
& =\frac{1}{L_{1} L_{2} L_{3}} \int_{0}^{L_{3}} \mathrm{e}^{-\mathrm{i} \ell_{3} x_{3}} \int_{0}^{L_{2}} \mathrm{e}^{-\mathrm{i} \ell_{2} x_{2}} \int_{0}^{L_{1}} u \mathrm{e}^{-\mathrm{i} \ell_{1} x_{1}} d x_{1} d x_{2} d x_{3} .
\end{aligned}
$$

(iv) Maximum vorticity. Perhaps one of the most important quantities in the regularity theory of the Euler equations, the maximum vorticity

$$
\|\omega\|_{\infty}:=\|\omega\|_{L^{\infty}(D(1, L))}=\max _{(r, z) \in D(1, L)}|\omega(r, z)|
$$

is closely monitored in most Euler computations, and its convergence under mesh refinement is also frequently used as a "quality indicator" for the underlying numerical simulations.

(v) Conservation of circulation. In a more recent work [8], the relative change of the circulation

$$
\Gamma=\oint_{C} u \cdot d s
$$

around selected material curves $C$ is proposed as an "error indicator" for Euler computations. The idea is that, according to Kelvin's circulation theorem, the circulation around any closed material curve $C$ is conserved by an Euler flow, and hence the same should be expected for a numerical solution as well. While conservation of circulation is a physically important principle, its numerical confirmation is not always plausible, because it is not always clear how to choose the "representative" material curves $C$. In addition, it is generally not easy to follow a material curve in an Euler flow, since 
most such simulations are performed on Eulerian meshes while tracking a material curve requires the use of a Lagrangian mesh.

We argue that none of the above "quality indicators" is adequate for the purpose of singularity detection. Admittedly, energy, enstrophy, and circulation are all physically significant quantities, and without doubt they should all be accurately resolved in any "reasonable" Euler simulations. On the other hand, it is also important to realize that these quantities are global quantities and do not measure the accuracy of a numerical solution at any particular point or even in any particular subset of the computational domain. Since blowing-up solutions of the Euler equations must be characterized by rapidly growing vorticity [3], and in most cases such intense vorticity amplification is realized in spatial regions with rapidly collapsing support [38, 32], it is crucial that the accuracy of a numerically detected blowup candidate be measured by local error metrics such as the pointwise (sup-norm) error. When restricted to bounded domains, the pointwise error is stronger than any other global error metrics in the sense that the latter can be easily bounded in terms of the former, while the converse does not hold true in general. Consequently, the pointwise error provides the most stringent measure for the quality of a blowup candidate, both near the point of blowup and over the entire (bounded) computational domain.

Arguing in a similar manner, we see that neither energy spectrum nor maximum vorticity gives an adequate measure of error for a potentially blowing-up solution. On the one hand, the construction of an energy spectrum removes the phase information and reduces the dimension of the data from three to one, leaving only an incomplete picture of a solution and hence of its associated error. On the other hand, maximum vorticity, albeit significant in its own right, does not tell us anything about a solution except at the point where the vorticity magnitude attained its maximum.

In view of the above considerations, we shall gauge the quality of our Euler simulations at any fixed time instant $t$ using the sup-norm relative errors of the computed solutions $\left(u_{1}, \omega_{1}, \psi_{1}\right)$. More specifically, we shall estimate the error of a given solution, say $u_{1}$, by comparing it with a "reference solution," say $\hat{u}_{1}$, that is computed at the same time $t$ on a finer mesh. The reference solution $\hat{u}_{1}$ is first interpolated to the coarse mesh on which $u_{1}$ is defined. Then the maximum difference between the two solutions is computed and the result is divided by the maximum of $\left|\hat{u}_{1}\right|$ (measured on the finer mesh) to yield the desired relative error.

We check the accuracy of our computations in five steps.

4.3.1. Code validation on test problems. First, we apply the numerical method described in section 3 to a test problem with known exact solutions and artificially generated external forcing terms (Appendix C). The exact solutions are chosen to mimic the behavior of the blowing-up Euler solution computed from (2.1)(2.2), and numerical experiments on successively refined meshes confirm the 6th-order convergence of the overall method (Table 7 ).

4.3.2. Resolution study on transformed primitive variables. Second, we perform a resolution study on the actual solutions of problem (2.1)-(2.2) at various time instants $t$, up to the time $t=0.003505$ shortly before the simulations terminate. For each $256 k \times 256 k$ mesh except for the finest one, we compare the solution $\left(u_{1}, \omega_{1}, \psi_{1}\right)$ computed on this mesh with the reference solution $\left(\hat{u}_{1}, \hat{\omega}_{1}, \hat{\psi}_{1}\right)$ computed at the same time $t$ on the finer $[256(k+1)] \times[256(k+1)]$ mesh and compute the sup-norm relative error using the procedure described above. For each $256 k \times 256 k$ mesh except for the coarsest one, we also compute, for each error $e_{k}$ defined on this mesh, the numerical order of convergence 
TABLE 7

Sup-norm relative error and numerical order of convergence (see (4.2)) of the transformed primitive variables $\left(u_{1}, \omega_{1}, \psi_{1}\right)$, computed for a test problem with known exact solutions. The absolute size of each variable, measured on the finest $768 \times 768$ mesh, is indicated in the last row "Sup-norm" of the table.

\begin{tabular}{ccccccc}
\hline \multirow{2}{*}{ Mesh size } & \multicolumn{7}{c}{ Sup-norm relative error at $t=0.029$} \\
\cline { 2 - 7 } & $u_{1}$ & Order & $\omega_{1}$ & Order & $\psi_{1}$ & Order \\
\hline $128 \times 128$ & $1.2252 \times 10^{-4}$ & - & $6.2554 \times 10^{-5}$ & - & $4.7084 \times 10^{-1}$ & - \\
$256 \times 256$ & $2.9249 \times 10^{-6}$ & 5.39 & $4.6254 \times 10^{-7}$ & 7.08 & $2.5819 \times 10^{-3}$ & 7.51 \\
$384 \times 384$ & $2.6925 \times 10^{-7}$ & 5.88 & $1.8224 \times 10^{-8}$ & 7.98 & $2.2455 \times 10^{-4}$ & 6.02 \\
$512 \times 512$ & $4.8713 \times 10^{-8}$ & 5.94 & $1.9185 \times 10^{-9}$ & 7.83 & $3.9857 \times 10^{-5}$ & 6.01 \\
$640 \times 640$ & $1.3293 \times 10^{-8}$ & 5.82 & $3.1179 \times 10^{-10}$ & 8.14 & $1.0217 \times 10^{-5}$ & 6.10 \\
$768 \times 768$ & $4.4301 \times 10^{-9}$ & 6.03 & $8.8603 \times 10^{-11}$ & 6.90 & $3.6163 \times 10^{-6}$ & 5.70 \\
\hline Sup-norm & $1.0000 \times 10^{-6}$ & - & $4.8900 \times 10^{3}$ & - & $1.1036 \times 10^{-10}$ & - \\
\hline
\end{tabular}

$$
\beta_{k}=\log _{k /(k-1)}\left(\frac{e_{k-1}}{e_{k}}\right) .
$$

Here, the error $e_{k}$ is understood as a function of the (uniform) mesh spacing $h_{r}=$ $h_{z}=1 /(256 k)$ in the $\rho \eta$-space, and it is assumed to admit an asymptotic expansion in powers of $h_{r}$ and $h_{z}$. Under suitable regularity assumptions on the underlying exact solutions and with suitable choices of time steps, it can be shown that $\beta_{k}$ converges to its theoretical value (6 in this case) as $k \rightarrow \infty$.

The results of the resolution study on the primitive variables $\left(u_{1}, \omega_{1}, \psi_{1}\right)$ among the five mesh resolutions are summarized in Figure 5. To examine more closely the errors at the times when the solutions are about to "blow up," we also report in Table 8 the estimated sup-norm errors and numerical orders at $t=0.003505$. It can be observed from these results that, for small $t$, specifically for $t \lesssim 0.0015$, the solutions are well resolved even on the coarsest $1024 \times 1024$ mesh, and further increase in mesh size does not lead to further improvement of the sup-norm errors. For $0.0015 \lesssim t \lesssim$ 0.0033 , the errors first grow exponentially in time and then level off after $t \approx 0.0028$. The numerical orders estimated on this time interval roughly match their theoretical values 6 , confirming the full-order convergence of the computed solutions. For $t \gtrsim$ 0.0033 , the exponential growth of the sup-norm errors resumes at an accelerated pace, in correspondence with the strong, nonlinear amplifications of the underlying solutions observed in this stage. The numerical orders estimated for $u_{1}$ and $\omega_{1}$ decline slightly from 6 to 4, as a result of the rapidly growing discretization error in time (Figure 7 ), while the ones for $\psi_{1}$ increase slightly from 6 to 8 , thanks most likely to the superconvergence property of the B-spline based Poisson solver at grid points (section 3.2 ). Based on these observations, we conclude that the primitive variables computed on the finest two meshes have at least four significant digits up to and including the time $t=0.003505$ shortly before the singularity forms. To the best of our knowledge, this level of accuracy has never been observed in previous numerical studies (see also Table 15).

4.3.3. Resolution study on vorticity vector. Since the Beale-Kato-Majda criterion suggests that the vorticity vector $\omega$ controls the blowup of smooth Euler solutions, we next perform a resolution study on $\omega$ to see how well it is resolved in our computations. The procedure is almost identical to that described for the primitive variables $\left(u_{1}, \omega_{1}, \psi_{1}\right)$, except that the difference between a vorticity vector $\omega$ and its reference value $\hat{\omega}$ needs to be measured in a suitable vector norm. By choosing the 


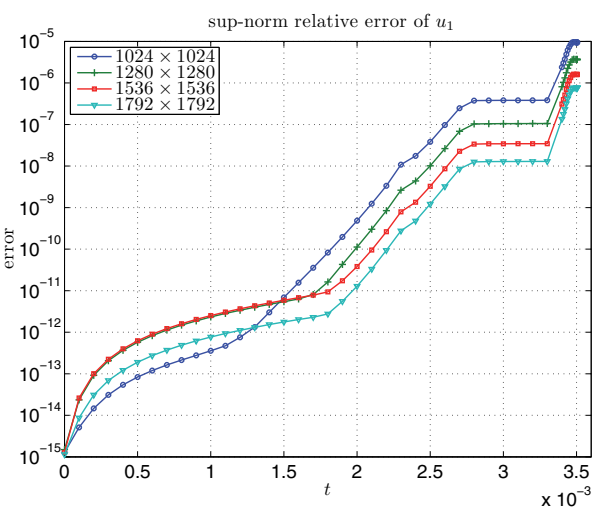

(a) sup-norm relative error of $u_{1}$

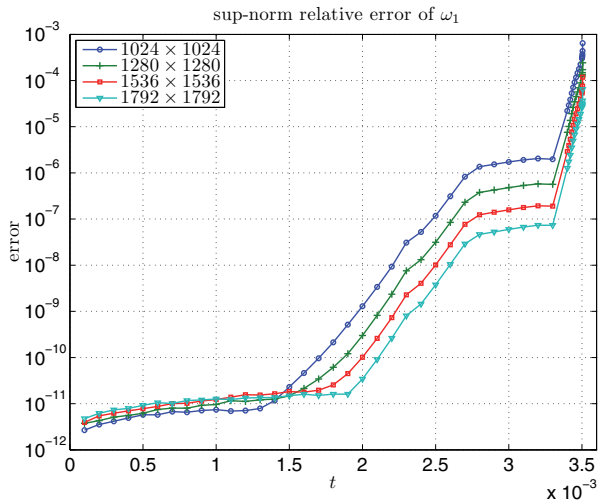

(c) sup-norm relative error of $\omega_{1}$

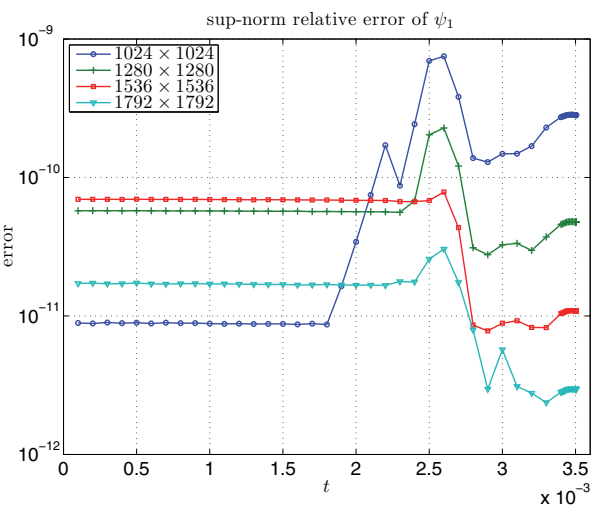

(e) sup-norm relative error of $\psi_{1}$

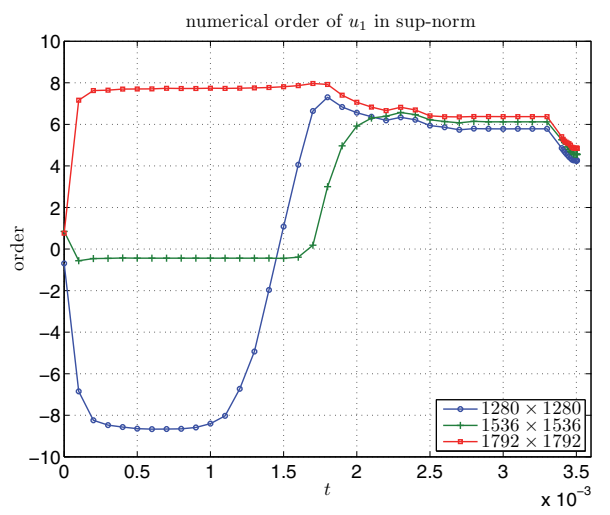

(b) numerical order of $u_{1}$ in sup-norm

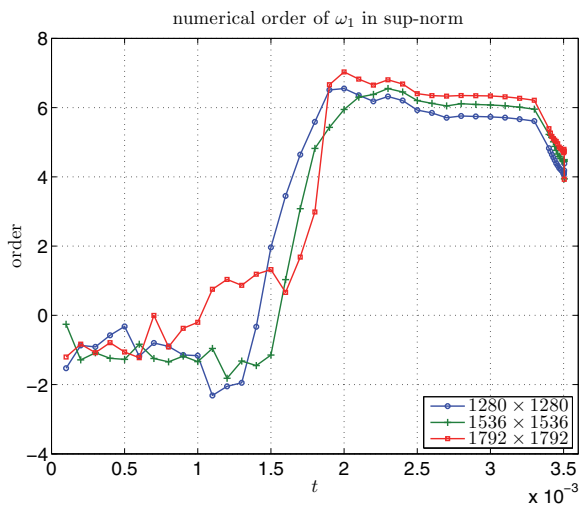

(d) numerical order of $\omega_{1}$ in sup-norm

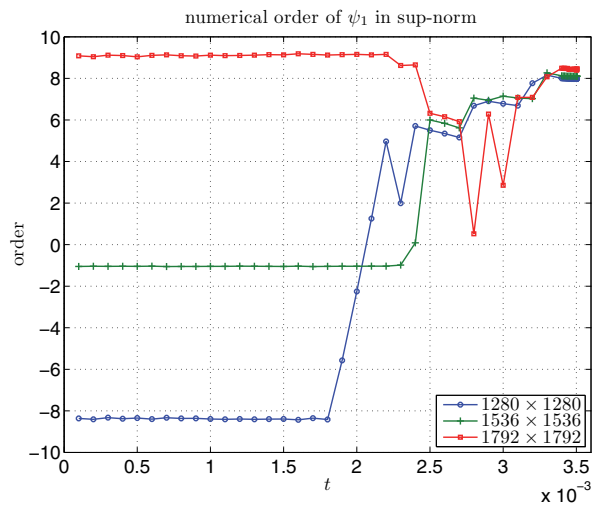

(f) numerical order of $\psi_{1}$ in sup-norm

FIG. 5. Resolution study in space: (a)(c)(e) sup-norm relative error and (b)(d)(f) numerical order in sup-norm of the transformed primitive variables $\left(u_{1}, \omega_{1}, \psi_{1}\right)$. The last time instant shown in the figure is $t=0.003505$.

usual Euclidean norm, we have

$$
\begin{aligned}
|\omega-\hat{\omega}| & =\left[\left(\omega^{r}-\hat{\omega}^{r}\right)^{2}+\left(\omega^{\theta}-\hat{\omega}^{\theta}\right)^{2}+\left(\omega^{z}-\hat{\omega}^{z}\right)^{2}\right]^{1 / 2} \\
& =\left[\left(r u_{1, z}-r \hat{u}_{1, z}\right)^{2}+\left(r \omega_{1, z}-r \hat{\omega}_{1, z}\right)^{2}+\left(2 u_{1}+r u_{1, r}-2 \hat{u}_{1}-r \hat{u}_{1, r}\right)^{2}\right]^{1 / 2} .
\end{aligned}
$$

Copyright (c) by SIAM. Unauthorized reproduction of this article is prohibited. 
TABLE 8

Sup-norm relative error and numerical order of convergence of the transformed primitive variables $\left(u_{1}, \omega_{1}, \psi_{1}\right)$ at $t=0.003505$. The absolute size of each variable, measured on the finest $2048 \times 2048$ mesh, is indicated in the last row "Sup-norm" of the table.

\begin{tabular}{ccccccc}
\hline \multirow{2}{*}{ Mesh size } & \multicolumn{6}{c}{ Sup-norm relative error at $t=0.003505$} \\
\cline { 2 - 7 } & $u_{1}$ & Order & $\omega_{1}$ & Order & $\psi_{1}$ & Order \\
\hline $1024 \times 1024$ & $9.4615 \times 10^{-6}$ & - & $6.4354 \times 10^{-4}$ & - & $2.8180 \times 10^{-10}$ & - \\
$1280 \times 1280$ & $3.6556 \times 10^{-6}$ & 4.26 & $2.4201 \times 10^{-4}$ & 4.38 & $4.7546 \times 10^{-11}$ & 7.97 \\
$1536 \times 1536$ & $1.5939 \times 10^{-6}$ & 4.55 & $1.1800 \times 10^{-4}$ & 3.94 & $1.0873 \times 10^{-11}$ & 8.09 \\
$1792 \times 1792$ & $7.5561 \times 10^{-7}$ & 4.84 & $6.4388 \times 10^{-5}$ & 3.93 & $2.9518 \times 10^{-12}$ & 8.46 \\
\hline Sup-norm & $1.0000 \times 10^{2}$ & - & $1.0877 \times 10^{6}$ & - & $2.1610 \times 10^{-1}$ & - \\
\hline
\end{tabular}

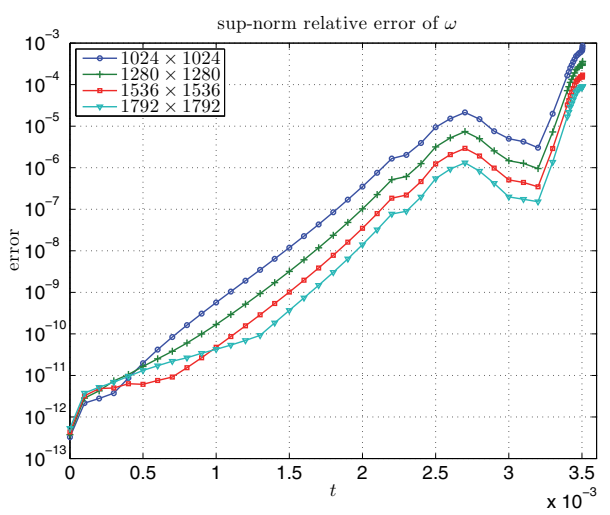

(a) sup-norm relative error

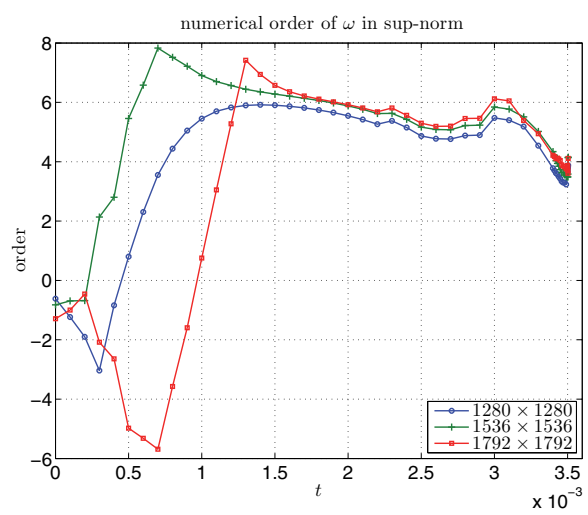

(b) numerical order in sup-norm

FIG. 6. Resolution study in space: (a) sup-norm relative error and (b) numerical order in sup-norm of the vorticity vector $\omega$. The last time instant shown in the figure is $t=0.003504$.

TABLE 9

Sup-norm relative error and numerical order of convergence of the vorticity vector $\omega$ at selected time $t$. The absolute size of $\omega$, measured on the finest $2048 \times 2048$ mesh, is indicated in the last row "Sup-norm" of the table.

\begin{tabular}{ccccc}
\hline \multirow{2}{*}{ Mesh size } & \multicolumn{4}{c}{ Sup-norm relative error of $\omega$} \\
\cline { 2 - 5 } & $t=0.003504$ & Order & $t=0.003505$ & Order \\
\hline $1024 \times 1024$ & $8.5671 \times 10^{-4}$ & - & $1.1352 \times 10^{-3}$ & - \\
$1280 \times 1280$ & $3.6084 \times 10^{-4}$ & 3.87 & $4.5801 \times 10^{-4}$ & 4.07 \\
$1536 \times 1536$ & $1.6929 \times 10^{-4}$ & 4.15 & $2.3050 \times 10^{-4}$ & 3.77 \\
$1792 \times 1792$ & $8.9837 \times 10^{-5}$ & 4.11 & $3.3212 \times 10^{-4}$ & $-^{\dagger}$ \\
\hline Sup-norm & $1.2209 \times 10^{11}$ & - & $1.2401 \times 10^{12}$ & - \\
\hline$\dagger:$ Round-off error begins to dominate.
\end{tabular}

The resulting sup-norm errors and numerical orders are summarized in Figure 6 and Table 9. These results will be used below in section 4.4 in the computation of the asymptotic scalings of the nearly singular solutions.

4.3.4. Resolution study on global quantities. The next step in our resolution study is to examine the "conventional" error indicators defined using global 
TABLE 10

Maximum (relative) change of kinetic energy $E$, minimum circulation $\Gamma_{1}$, and maximum circulation $\Gamma_{2}$ over the time interval [0,0.003505]. The initial value of each quantity, measured on the finest $2048 \times 2048$ mesh, is indicated in the last row "Init. value" of the table.

\begin{tabular}{cccc}
\hline \multirow{2}{*}{ Mesh size } & \multicolumn{3}{c}{$t=0.003505$} \\
\cline { 2 - 4 } & $\|\delta E\|_{\infty, t}$ & $\left\|\delta \Gamma_{1}\right\|_{\infty, t}$ & $\left\|\delta \Gamma_{2}\right\|_{\infty, t}$ \\
\hline $1024 \times 1024$ & $1.5259 \times 10^{-11}$ & $4.3525 \times 10^{-17}$ & $1.2485 \times 10^{-14}$ \\
$1280 \times 1280$ & $4.1730 \times 10^{-12}$ & $3.3033 \times 10^{-17}$ & $7.7803 \times 10^{-15}$ \\
$1536 \times 1536$ & $2.0787 \times 10^{-12}$ & $3.1308 \times 10^{-17}$ & $9.9516 \times 10^{-15}$ \\
$1792 \times 1792$ & $6.4739 \times 10^{-13}$ & $2.7693 \times 10^{-17}$ & $2.1351 \times 10^{-14}$ \\
$2048 \times 2048$ & $6.6594 \times 10^{-13}$ & $2.5308 \times 10^{-17}$ & $3.4921 \times 10^{-14}$ \\
\hline Init. value & 55.9309 & 0.0000 & $6.2832 \times 10^{2}$ \\
\hline
\end{tabular}

quantities such as energy $E$, enstrophy $\mathcal{E}$, enstrophy production rate $\mathcal{E}_{p},{ }^{5}$ maximum vorticity $\|\omega\|_{\infty},{ }^{6}$ and circulation $\Gamma$. As we already pointed out, conservation of circulation is physically important but is difficult to check in practice, because it requires selection and tracking of representative material curves, which is not always easy. On the other hand, in axisymmetric flows the total circulation along the circular contours

$$
C=\left\{(x, y, z) \in \mathbb{R}^{3}: x^{2}+y^{2}=r^{2}<1, z \text { a constant }\right\}
$$

is easily found to be $\Gamma=2 \pi r^{2} u_{1}$. Thus as an alternative to conservation of circulation, we choose to monitor the extreme circulations

$$
\Gamma_{1}=2 \pi \min _{(r, z) \in D(1, L)} r^{2} u_{1}(r, z), \quad \Gamma_{2}=2 \pi \max _{(r, z) \in D(1, L)} r^{2} u_{1}(r, z),
$$

which must be conserved over time according to Kelvin's circulation theorem.

We study the errors of the above-mentioned global quantities as follows. For conserved quantities such as kinetic energy and extreme circulations, the maximum (relative) change

$$
\|\delta Q\|_{\infty, t}=\max _{s \in[0, t]}|\delta Q(s)|
$$

over the time interval $[0, t]$ is computed, where

$$
\delta Q(t)= \begin{cases}Q(0)^{-1}[Q(t)-Q(0)] & \text { if } Q(0) \neq 0 \\ Q(t)-Q(0) & \text { if } Q(0)=0\end{cases}
$$

For other nonconservative quantities, the relative error

$$
\frac{1}{\hat{Q}(t)}|Q(t)-\hat{Q}(t)|
$$

is computed where $Q$ denotes global quantities computed on a $256 k \times 256 k$ mesh and $\hat{Q}$ represents reference values obtained on the finer $[256(k+1)] \times[256(k+1)]$ mesh. The resulting errors and numerical orders at $t=0.003505$ are summarized in Tables $10-11$.

\footnotetext{
${ }^{5}$ All these integrals are discretized in the $\rho \eta$-space using the 6th-order composite Boole's rule.

${ }^{6}$ We define $\|\omega\|_{\infty}$ simply as the maximum value of $|\omega|$ on the discrete mesh points (i.e., no interpolation is used to find the "precise" maximum). In view of the highly effective adaptive mesh, this does not cause any loss of accuracy. In addition, for the specific initial condition $(2.2 \mathrm{a}),\|\omega\|_{\infty}$ is always attained at $\tilde{q}_{0}=(1,0)^{T}$, which is always a mesh point.
} 
TABLE 11

Relative error of enstrophy $\mathcal{E}$, enstrophy production rate $\mathcal{E}_{p}$, and maximum vorticity $\|\omega\|_{\infty}$ at $t=0.003505$. The absolute size of each quantity, measured on the finest $2048 \times 2048$ mesh, is indicated in the last row "Ref. value" of the table.

\begin{tabular}{|c|c|c|c|c|c|c|}
\hline \multirow{2}{*}{ Mesh size } & \multicolumn{6}{|c|}{ Relative error at $t=0.003505$} \\
\hline & $\mathcal{E}$ & Order & $\mathcal{E}_{p}$ & Order & $\|\omega\|_{\infty}$ & Order \\
\hline $1024 \times 1024$ & $4.6075 \times 10^{-6}$ & - & $4.6565 \times 10^{-5}$ & - & $7.7593 \times 10^{-4}$ & - \\
\hline $1280 \times 1280$ & $1.4946 \times 10^{-6}$ & 5.05 & $1.4488 \times 10^{-5}$ & 5.23 & $3.0099 \times 10^{-4}$ & 4.24 \\
\hline $1536 \times 1536$ & $5.6161 \times 10^{-7}$ & 5.37 & $5.3275 \times 10^{-6}$ & 5.49 & $1.2927 \times 10^{-4}$ & 4.64 \\
\hline $1792 \times 1792$ & $2.3385 \times 10^{-7}$ & 5.68 & $2.0314 \times 10^{-6}$ & 6.25 & $6.1010 \times 10^{-5}$ & 4.87 \\
\hline Ref. value & $7.0254 \times 10^{5}$ & - & $1.4270 \times 10^{10}$ & - & $1.2401 \times 10^{12}$ & - \\
\hline
\end{tabular}

As a side remark, we note that the error of the maximum vorticity $\|\omega\|_{\infty}$ is always a lower bound of the error of the vorticity vector $\omega$. This is a direct consequence of the triangle inequality

$$
\left|\|\omega\|_{\infty}-\|\hat{\omega}\|_{\infty}\right| \leq\|\omega-\hat{\omega}\|_{\infty}
$$

and is readily confirmed by the results shown in Tables 9 and 11. In addition, note that global errors such as the error of the enstrophy $\mathcal{E}$ can significantly underestimate the pointwise error of the vorticity vector $\omega$. This confirms the inadequacy of the "conventional" error indicators in the context of singularity detection.

4.3.5. Resolution study in time. Finally, we perform a resolution study in time by repeating the $1792 \times 1792$ mesh computation using smaller time steps $\delta_{t}$. This is achieved by reducing the CFL number from $\nu=0.5$ to $0.4,0.3$ and the relative growth threshold from $\epsilon_{t}=5 \%$ to $4 \%, 3 \%$ (section 3.3). For each reduced time step computation, the resulting solution $\left(\hat{u}_{1}, \hat{\omega}_{1}, \hat{\psi}_{1}, \hat{\omega}\right)$ is taken as the reference solution and is compared with the original solution $\left(u_{1}, \omega_{1}, \psi_{1}, \omega\right)$ computed using $\left(\nu, \epsilon_{t}\right)=$ $(0.5,5 \%)$. The corresponding sup-norm errors are summarized in Figure 7 and Table 12. Note that the error between the computations $\left(\nu, \epsilon_{t}\right)=\{(0.3,3 \%),(0.5,5 \%)\}$ is roughly the same as that between the computations $\left(\nu, \epsilon_{t}\right)=\{(0.4,4 \%),(0.5,5 \%)\}$, which is smaller than the error between the $1792 \times 1792$ and the $2048 \times 2048$ mesh computations. This indicates that the solutions computed on the $1792 \times 1792$ and all the coarser meshes with $\left(\nu, \epsilon_{t}\right)=(0.5,5 \%)$ are well resolved in time up to $t=0.003505$.

4.4. Asymptotic scaling analysis I: Maximum vorticity. With the pointwise error bounds derived in the previous section, we are ready to examine the numerical data in greater detail and apply the mathematical criteria reviewed in section 1 to assess the likelihood of a finite-time singularity.

The basic tool that we shall use is the well-known Beale-Kato-Majda (BKM) criterion [3]. According to this criterion, a smooth solution of the 3D Euler equations blows up at time $t_{s}$ if and only if

$$
\int_{0}^{t_{s}}\|\omega(\cdot, t)\|_{\infty} d t=\infty
$$

where $\|\omega(\cdot, t)\|_{\infty}$ is the maximum vorticity at time $t$. The BKM criterion was originally proved in [3] for flows in free space $\mathbb{R}^{3}$ and was later generalized by $[22,46]$ to flows in smooth bounded domains subject to no-flow boundary conditions. In view of this criterion, a "standard" approach to singularity detection in Euler computations is to 


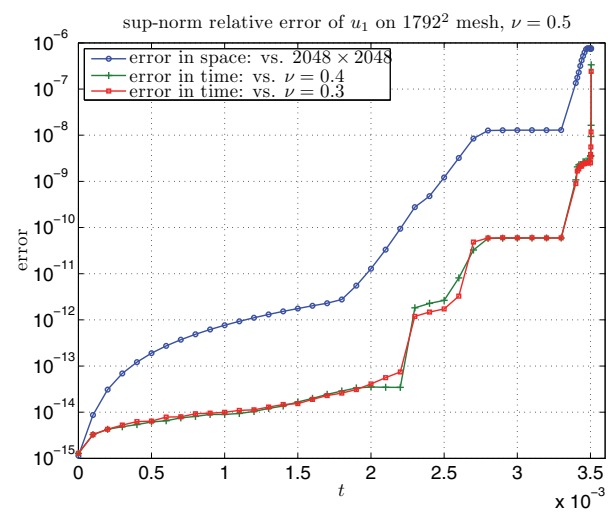

(a) sup-norm relative error of $u_{1}$

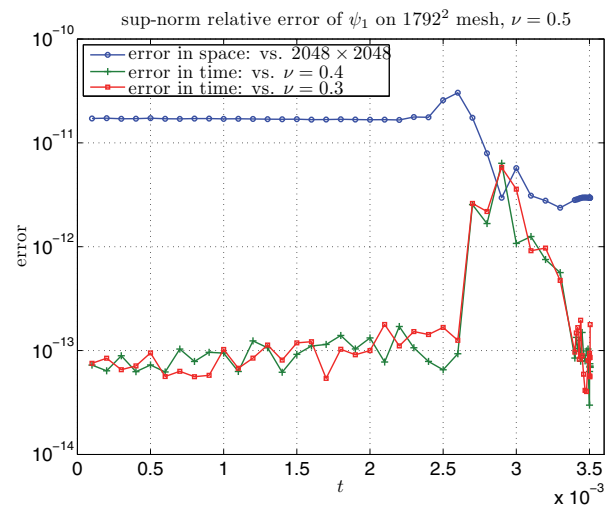

(c) sup-norm relative error of $\psi_{1}$

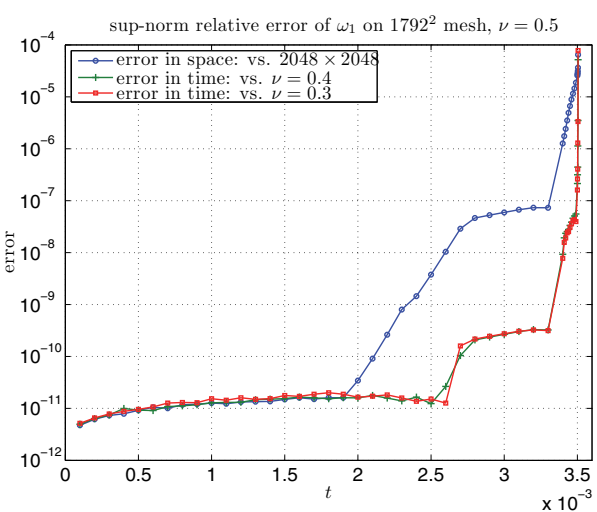

(b) sup-norm relative error of $\omega_{1}$

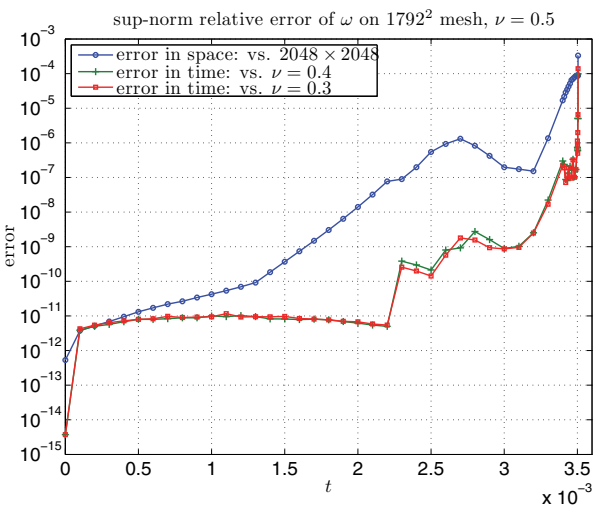

(d) sup-norm relative error of $\omega$

FIG. 7. Resolution study in time: sup-norm relative error of (a)-(c) the transformed primitive variables $\left(u_{1}, \omega_{1}, \psi_{1}\right)$ and $(\mathrm{d})$ the vorticity vector $\omega$, computed on the $1792 \times 1792$ mesh. The last time instant shown in the figure is $t=0.003505$.

TABLE 12

Sup-norm relative error of the transformed primitive variables $\left(u_{1}, \omega_{1}, \psi_{1}\right)$ and the vorticity vector $\omega$, computed on the $1792 \times 1792$ mesh with $\left(\nu, \epsilon_{t}\right)=(0.5,5 \%)$ and compared with different reference solutions. The absolute size of each variable, measured on the finest $2048 \times 2048$ mesh, is indicated in the last row "Sup-norm" of the table.

\begin{tabular}{ccccc}
\hline \multirow{2}{*}{ Ref. solution } & \multicolumn{4}{c}{ Sup-norm relative error at $t=0.003505$} \\
\cline { 2 - 5 } & $u_{1}$ & $\omega_{1}$ & $\psi_{1}$ & $\omega$ \\
\hline $2048 \times 2048$ & $7.5561 \times 10^{-7}$ & $6.4388 \times 10^{-5}$ & $2.9518 \times 10^{-12}$ & $3.3212 \times 10^{-4}$ \\
$\left(\nu, \epsilon_{t}\right)=(0.4,4 \%)$ & $3.3350 \times 10^{-7}$ & $5.1609 \times 10^{-5}$ & $6.8713 \times 10^{-14}$ & $9.7514 \times 10^{-5}$ \\
$\left(\nu, \epsilon_{t}\right)=(0.3,3 \%)$ & $2.4197 \times 10^{-7}$ & $7.7720 \times 10^{-5}$ & $1.7776 \times 10^{-13}$ & $1.3800 \times 10^{-4}$ \\
\hline Sup-norm & $1.0000 \times 10^{2}$ & $1.0877 \times 10^{6}$ & $2.1610 \times 10^{-1}$ & $1.2401 \times 10^{12}$ \\
\hline
\end{tabular}

assume the existence of an appropriate asymptotic scaling for $\|\omega\|_{\infty}$, typically in the form of an inverse power-law

$$
\|\omega(\cdot, t)\|_{\infty} \sim c\left(t_{s}-t\right)^{-\gamma}, \quad c, \gamma>0
$$

Copyright (C) by SIAM. Unauthorized reproduction of this article is prohibited. 
then an estimate of the (unknown) singularity time $t_{s}$ and the scaling parameters $(c, \gamma)$ is obtained from a line fitting procedure. Normally, the line fitting is computed on some interval $\left[\tau_{1}, \tau_{2}\right]$ prior to the predicted singularity time $t_{s}$, and the results are extrapolated forward in time to yield the desired estimates.

Although seemingly straightforward, the above procedure must be used with caution. Indeed, there are examples where inadvertent line fitting has led to false predictions of finite-time singularities. As we shall demonstrate below, the key to the successful application of the line fitting procedure lies in the choice of the fitting interval $\left[\tau_{1}, \tau_{2}\right]$. One must realize, upon the invocation of (4.3), that the applicability of this form fit is not known a priori and must be determined from the line fitting itself. In order for the line fitting to work, the interval $\left[\tau_{1}, \tau_{2}\right]$ must be placed within the asymptotic regime of (4.3) if scalings of that form do exist. If such an asymptotic regime cannot be identified, then the validity of (4.3) is questionable and any conclusions drawn from the line fitting are likely to be false.

In most existing studies, the choice of the fitting interval $\left[\tau_{1}, \tau_{2}\right]$ is based on discretionary manual selections, which tend to generate results that lack clear interpretations and are difficult to reproduce. To overcome these difficulties, we propose choosing $\tau_{1}, \tau_{2}$ using an automatic procedure which in ideal situations should place $\tau_{2}$ at $t_{s}$ and $\tau_{1}$ at a point "close enough" to $t_{s}$, in such a way that $\left[\tau_{1}, \tau_{2}\right]$ is enclosed in the asymptotic regime of (4.3). In reality, such a choice can never be made, because a singularity time $t_{s}$, if it exists, can never be attained by a numerical simulation. Thus we propose placing $\tau_{1}, \tau_{2}$ close enough to the stopping time $t_{e}$ such that the computed solutions are still "well resolved" on $\left[\tau_{1}, \tau_{2}\right]$ and an asymptotic scaling of the form (4.3) exists and dominates in $\left[\tau_{1}, \tau_{2}\right]$. To this end, we shall choose $\tau_{2}$ to be the first time instant at which the sup-norm relative error of the vorticity vector $\omega$ exceeds a certain threshold $\epsilon_{2}$ and choose $\tau_{1}$ so that $\left[\tau_{1}, \tau_{2}\right]$ is the interval on which the line fitting yields the "best" results (in a sense to be made precise below). Note that the accuracy of the computed solutions is measured in terms of the error of $\omega$, not that of $\left(u_{1}, \omega_{1}, \psi_{1}\right)$, because $\omega$ is the quantity that controls the blowup.

We consider a line fitting "successful" if both $\tau_{2}$ and the line-fitting predicted singularity time $\hat{t}_{s}$ converge to the same finite value as the mesh is refined. The convergence should be monotone, i.e., $\tau_{2} \uparrow t_{s}, \hat{t}_{s} \downarrow t_{s}$, where $t_{s}$ is the common limit, the true singularity time. In addition, $\tau_{1}$ should converge to a finite value that is strictly less than $t_{s}$ as the mesh is refined. The reason that the convergence of $\tau_{2}, \hat{t}_{s}$ to the singularity time $t_{s}$ should be monotone is two-fold: first, the finer the mesh, the longer it takes the error to grow to a given tolerance, and hence the larger the $\tau_{2}$ is; second, as $\tau_{2}$ gets increasingly closer to $t_{s}$, the strong, singular growth of the blowing-up solution is better captured on $\left[\tau_{1}, \tau_{2}\right]$, which then translates into an earlier estimate $\hat{t}_{s}$ of the blowup time.

If the interval $\left[\tau_{1}, \tau_{2}\right]$ can be chosen to satisfy all the above criteria, and the scaling parameters $(c, \gamma)$ estimated on this interval converge to some finite values $c_{s}>0, \gamma_{s} \geq 1$ as the mesh is refined, then the existence of a finite-time singularity is confirmed.

Let us now apply these ideas to our numerical data.

4.4.1. The line fitting procedure. We first describe a line fitting procedure that will be needed in both the choice of the fitting interval $\left[\tau_{1}, \tau_{2}\right]$ (section 4.4.2) and the computation of the asymptotic scaling (4.3). Under the assumption that the maximum vorticity $\|\omega\|_{\infty}$ is approximated sufficiently well by the inverse powerlaw (4.3) on the interval $\left[\tau_{1}, \tau_{2}\right]$, the logarithmic time derivative, or simply the log 
$t$-derivative, of $\|\omega\|_{\infty}$ is easily found to satisfy

$$
\frac{d}{d t} \log \|\omega(\cdot, t)\|_{\infty}=\|\omega(\cdot, t)\|_{\infty}^{-1} \frac{d}{d t}\|\omega(\cdot, t)\|_{\infty} \sim \frac{\gamma}{t_{s}-t} .
$$

This leads to the simple linear regression model

$$
y(t):=\left[\frac{d}{d t} \log \|\omega(\cdot, t)\|_{\infty}\right]^{-1} \sim-\frac{1}{\gamma}\left(t-t_{s}\right)=: a t+b,
$$

with response variable $y$, explanatory variable $t$, and model parameters $a=-1 / \gamma, b=$ $t_{s} / \gamma$. The model parameters in (4.4) can be estimated from a standard least-squares procedure. The fitness of the model can be measured using either the coefficient of determination (the $R^{2}$ ),

$$
R^{2}=1-\frac{S S_{\mathrm{err}}}{S S_{\mathrm{tot}}}
$$

where a value close to 1 indicates good fitness, or the fraction of variance unexplained (FVU),

$$
\mathrm{FVU}=1-R^{2}=\frac{S S_{\mathrm{err}}}{S S_{\mathrm{tot}}}
$$

where a value close to 0 indicates good fitness. Here

$$
S S_{\mathrm{tot}}=\sum_{i}\left(y_{i}-\bar{y}\right)^{2}
$$

is the total sum of squares and

$$
S S_{\mathrm{err}}=\sum_{i}\left(y_{i}-\hat{y}_{i}\right)^{2}
$$

is the residual sum of squares, where $y_{i}, \hat{y}_{i}$ denote the observed and predicted values of the response variable $y$, respectively, and $\bar{y}$ denotes the mean of the observed data $y_{i}$.

To apply the above line fitting procedure to our numerical data, we need the time derivative of the maximum vorticity, $\frac{d}{d t}\|\omega\|_{\infty}$. For the specific initial condition $(2.2 \mathrm{a})$, the maximum vorticity is always attained at the corner $\tilde{q}_{0}=(1,0)^{T}$. Due to the special symmetry properties of the solution (section 2) and the no-flow boundary condition $(2.2 \mathrm{c})$, the vorticity vector $\omega$ at $\tilde{q}_{0}$ has a particularly simple form:

$$
\omega\left(\tilde{q}_{0}\right)=\left.\left(-r u_{1, z}, r \omega_{1}, 2 u_{1}+r u_{1, r}\right)^{T}\right|_{\tilde{q}_{0}}=\left(-u_{1, z}\left(\tilde{q}_{0}\right), 0,0\right)^{T} .
$$

Consequently, the time derivative and the $\log t$-derivative of the maximum vorticity can be readily computed as

$$
\frac{d}{d t}\|\omega(\cdot, t)\|_{\infty}=\frac{d}{d t}\left|\tilde{u}_{1, z}\right|=-\tilde{\psi}_{1, r z}\left|\tilde{u}_{1, z}\right|, \quad \frac{d}{d t} \log \left[\|\omega(\cdot, t)\|_{\infty}^{-1}\right]=\tilde{\psi}_{1, r z},
$$

where for simplicity we have written $\tilde{u}_{1, z}=u_{1, z}\left(\tilde{q}_{0}\right)$ and $\tilde{\psi}_{1, r z}=\psi_{1, r z}\left(\tilde{q}_{0}\right)$.

Once an estimate $\hat{t}_{s}$ of the singularity time $t_{s}$ is obtained, the scaling parameter $c$ in (4.3) can be determined from another linear regression problem:

$$
\tilde{y}(\tilde{t}):=\log \|\omega(\cdot, t)\|_{\infty} \sim-\gamma \log \left(\hat{t}_{s}-t\right)+\log c=: \tilde{a} \tilde{t}+\tilde{b},
$$

where $\tilde{y}$ is the response variable, $\tilde{t}=\log \left(\hat{t}_{s}-t\right)$ is the explanatory variable, and $\tilde{a}=-\gamma, \tilde{b}=\log c$ are model parameters. As before, the model parameters in (4.6) can be estimated from a standard least-squares procedure, and the fitness of the model can be measured using either the $R^{2}$ or the FVU.

Copyright $@$ by SIAM. Unauthorized reproduction of this article is prohibited. 


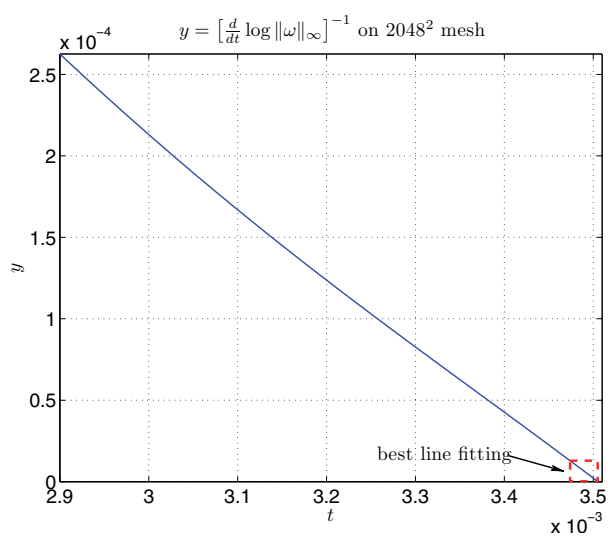

FIG. 8. Inverse log $t$-derivative of the maximum vorticity computed on the $2048 \times 2048$ mesh. The dashed line box represents the best fitting interval $\left[\tau_{1}, \tau_{2}\right]$.

4.4.2. Determination of $\tau_{1}$ and $\tau_{2}$. With the above line fitting procedure, we are now ready to describe the algorithm for choosing the fitting interval $\left[\tau_{1}, \tau_{2}\right]$.

The first step of the algorithm is to determine $\tau_{2}$, which is formally defined to be the first time instant at which the sup-norm relative error of the vorticity vector $\omega$ exceeds a certain threshold $\epsilon_{2}$. Note that this definition of $\tau_{2}$ needs to be modified on the finest $2048 \times 2048$ mesh, because the error of $\omega$ is not available there. In what follows, we shall define the value of $\tau_{2}$ on the $2048 \times 2048$ mesh to be the same as that computed on the $1792 \times 1792$ mesh. This is reasonable given that the error computed on the $1792 \times 1792$ mesh is likely an overestimate of the error computed on the $2048 \times 2048$ mesh, as indicated by the resolution study in section 4.3 .3 where convergence of $\omega$ under mesh refinement is observed.

Once $\tau_{2}$ is known, the next step of the algorithm is to determine $\tau_{1}$, which is formally defined to be the time instant at which the FVU of the line fitting computed on $\left[\tau_{1}, \tau_{2}\right]$ attains its minimum. To avoid placing too few or too many points in $\left[\tau_{1}, \tau_{2}\right]$, which may lead to line fittings with too much noise or too much bias, we choose $\tau_{1}$ in such a way that $\tau_{1} \leq \tau_{2}-\epsilon_{1}$ for some $\epsilon_{1}>0$ and the FVU of the line fitting computed on $\left[t, \tau_{2}\right]$, when viewed as a function of $t$, attains a local (instead of global) minimum in a neighborhood of $\tau_{1}$.

4.4.3. Evidence for finite-time blowup. We now apply the line fitting procedure described in sections 4.4.1-4.4.2 to our numerical data to assess the likelihood of a finite-time singularity. As demonstrated earlier in section 4.2, the maximum vorticity $\|\omega\|_{\infty}$ computed from (2.1)-(2.2) has a growth rate faster than double-exponential (Figure 4). To see whether $\|\omega\|_{\infty}$ blows up in finite time, we plot in Figure 8 the inverse $\log t$-derivative of the maximum vorticity (see (4.4))

$$
y(t)=\left[\frac{d}{d t} \log \|\omega(\cdot, t)\|_{\infty}\right]^{-1}
$$

computed on the $2048 \times 2048$ mesh. Intuitively, the inverse log $t$-derivative approaches a straight line after $t \approx 0.0032$, which suggests that the maximum vorticity indeed admits an inverse power-law of the form (4.3). Motivated by this observation, we apply the line fitting to the data $y$ and report the resulting estimates in Table 13. It can be observed from this table that all estimated parameters converge to a finite 
TABLE 13

The best line fittings (4.4) and (4.6) computed on the interval $\left[\tau_{1}, \tau_{2}\right]$ with $n$ data points.

\begin{tabular}{cccccccc}
\hline Mesh size & $n$ & $\tau_{1}$ & $\tau_{2}$ & $\hat{t}_{s}$ & $\hat{\gamma}_{1}$ & $\hat{\gamma}_{2}$ & $\hat{c}$ \\
\hline $1024 \times 1024$ & 58 & 0.003306 & 0.003410 & 0.0035070 & 2.5041 & 2.5062 & $4.8293 \times 10^{-4}$ \\
$1280 \times 1280$ & 47 & 0.003407 & 0.003453 & 0.0035063 & 2.4866 & 2.4894 & $5.5362 \times 10^{-4}$ \\
$1536 \times 1536$ & 20 & 0.003486 & 0.003505 & 0.0035056 & 2.4544 & 2.4559 & $7.4912 \times 10^{-4}$ \\
$1792 \times 1792$ & 27 & 0.003479 & 0.003505 & 0.0035056 & 2.4557 & 2.4566 & $7.4333 \times 10^{-4}$ \\
$2048 \times 2048$ & 32 & 0.003474 & 0.003505 & 0.0035056 & 2.4568 & 2.4579 & $7.3273 \times 10^{-4}$ \\
\hline
\end{tabular}

limit as the mesh is refined, where in particular both $\tau_{2}$ and $\hat{t}_{s}$ tend to a common limit in a monotonic fashion. ${ }^{7}$ In addition, it can be seen that the limit of $\tau_{1}$ is strictly less than the common limit of $\tau_{2}$ and $\hat{t}_{s}$, indicating the existence of an asymptotic regime. Moreover, both estimates $\hat{\gamma}_{1}, \hat{\gamma}_{2}$ of $\gamma$ (computed from (4.4) and (4.6), respectively) approach a common limit with a value close to $\frac{5}{2} \geq 1$, and the limit of $\hat{c}$ is strictly positive. Based on these observations and the BKM criterion, we conclude that the solution of problem (2.1)-(2.2) develops a singularity at $t_{s} \approx 0.0035056$.

It is interesting to compare at this point the two estimates $\hat{\gamma}_{1}, \hat{\gamma}_{2}$ of the scaling exponent $\gamma$ computed from the line fitting problems (4.4) and (4.6). As can be observed from Table 13, the estimate $\hat{\gamma}_{2}$ computed from (4.6) is always slightly larger than that $\hat{\gamma}_{1}$ computed from (4.4). This is expected, because the singularity time $\hat{t}_{s}$ estimated from (4.4) decreases monotonically as the mesh is refined, indicating that $\hat{t}_{s}$ is always an overestimate of the true singularity time $t_{s}$. Consequently, the inverse power-law $\left(\hat{t}_{s}-t\right)^{-\gamma}$ necessarily underestimates the maximum vorticity $\|\omega\|_{\infty} \sim\left(t_{s}-\right.$ $t)^{-\gamma}$ when $t$ is sufficiently close to $t_{s}$, and the scaling exponent $\hat{\gamma}_{2}$ estimated from (4.6) has to be artificially magnified to compensate for this discrepancy. This explains the larger value of $\hat{\gamma}_{2}$ compared with $\hat{\gamma}_{1}$.

The computation of $\hat{\gamma}_{1}$ from (4.4), on the other hand, does not suffer from this problem and is expected to yield a more accurate estimate of $\gamma$. Thus in what follows we shall always choose $\hat{\gamma}_{1}$ as the estimated value of $\gamma$.

To measure the quality of the line fittings computed in Table 13, we introduce the "extrapolated FVU,"

$$
\mathrm{FVU}_{\mathrm{e}}=\frac{S S_{\mathrm{e}, \mathrm{err}}}{S S_{\mathrm{e}, \mathrm{tot}}}
$$

where $S S_{\text {e,tot }}$ and $S S_{\text {e,err }}$ are the total sum of squares and residual sum of squares defined on the extrapolation interval $\left[\tau_{2}, t_{e}\right]$, respectively. These extrapolated FVU, together with the FVU computed on $\left[\tau_{1}, \tau_{2}\right]$, are summarized below in Table 14 . We also plot in Figure 9 the maximum vorticity $\|\omega\|_{\infty}$, the inverse $\log t$-derivative of $\|\omega\|_{\infty}$, and their corresponding form fit computed on the $2048 \times 2048$ mesh. It can be observed from these results that both linear models (4.4) and (4.6) fit the data very well, as clearly indicated by the very small values of FVU. In addition, the line fittings provide an excellent approximation to the data even in the extrapolation interval, as the small values of $\mathrm{FVU}_{\mathrm{e}}$ show. Based on these observations, we conclude that the estimates obtained in Table 13 are trustworthy.

\footnotetext{
${ }^{7}$ The small discrepancy between the limits of $\tau_{2}$ and $\hat{t}_{s}$ is due to the fact that the sup-norm errors of $\omega$ are computed only at a discrete set of time instants. This restricts the definition of $\tau_{2}$ to a discrete set of values.
} 
TABLE 14

The FVU and FVUe of the line fittings (4.4) and (4.6).

\begin{tabular}{ccccc}
\hline Mesh size & FVU of $(4.4)$ & FVU $_{\mathrm{e}}$ of $(4.4)$ & FVU of $(4.6)$ & FVU $_{\mathrm{e}}$ of $(4.6)$ \\
\hline $1024 \times 1024$ & $8.7255 \times 10^{-7}$ & $6.1426 \times 10^{-4}$ & $1.9901 \times 10^{-8}$ & $1.0657 \times 10^{-1}$ \\
$1280 \times 1280$ & $3.3648 \times 10^{-6}$ & $6.2433 \times 10^{-4}$ & $3.0463 \times 10^{-8}$ & $7.9442 \times 10^{-2}$ \\
$1536 \times 1536$ & $2.4372 \times 10^{-7}$ & $6.0014 \times 10^{-4}$ & $4.1369 \times 10^{-7}$ & $1.0409 \times 10^{-3}$ \\
$1792 \times 1792$ & $1.0127 \times 10^{-7}$ & $4.5958 \times 10^{-4}$ & $2.4588 \times 10^{-7}$ & $8.0410 \times 10^{-4}$ \\
$2048 \times 2048$ & $9.3767 \times 10^{-8}$ & $1.0956 \times 10^{-4}$ & $2.8074 \times 10^{-8}$ & $1.6966 \times 10^{-4}$ \\
\hline
\end{tabular}

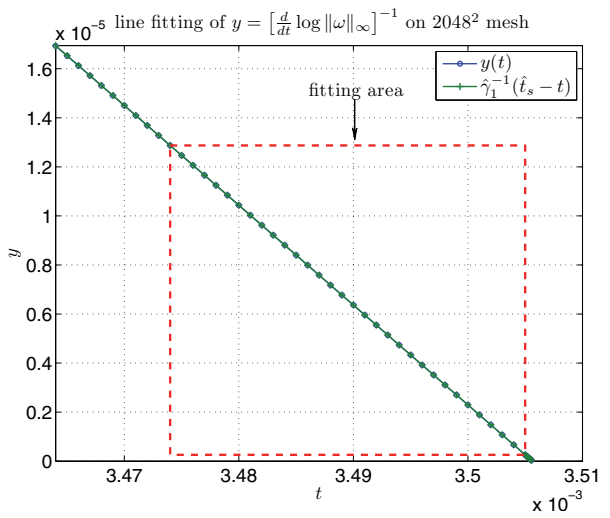

(a) line fitting (4.4)

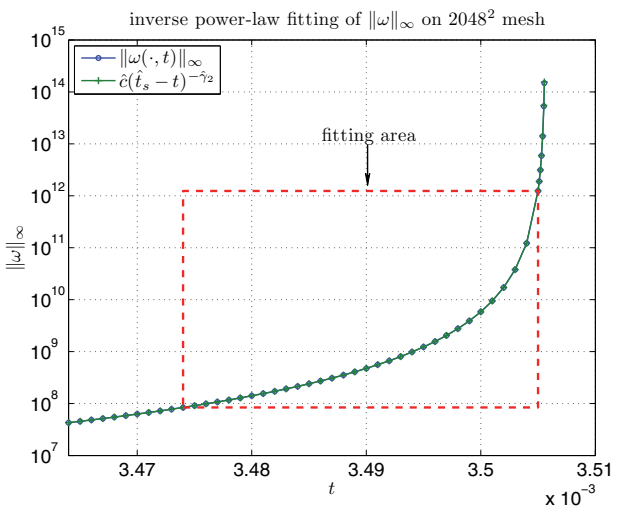

(c) line fitting (4.6)

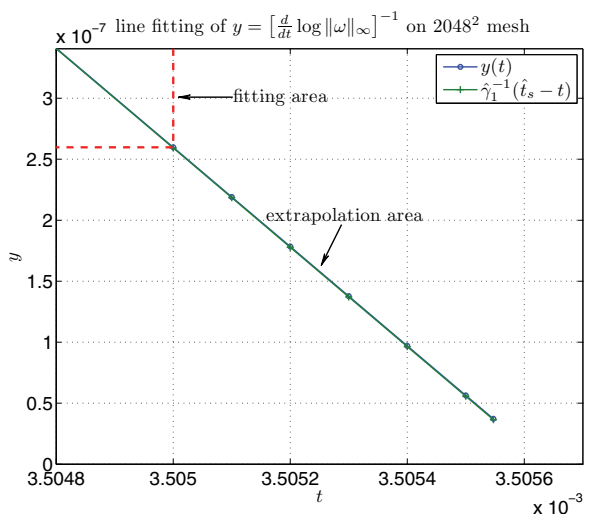

(b) line fitting (4.4) (zoom-in)

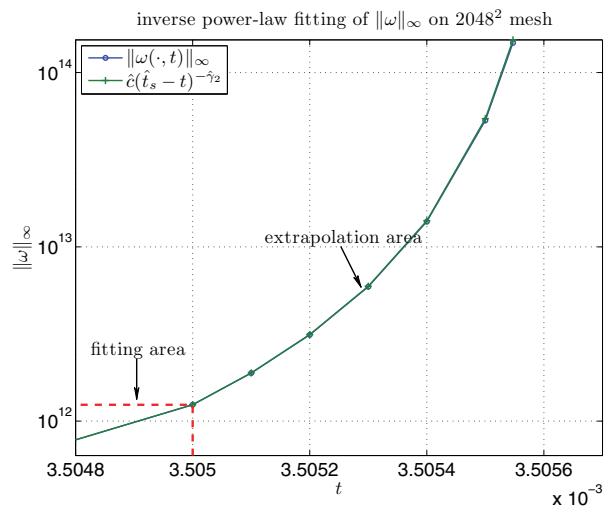

(d) line fitting (4.6) (zoom-in)

FIG. 9. Blowup of the maximum vorticity: (a) inverse log $t$-derivative of $\|\omega\|_{\infty}$ and its line fit $\hat{\gamma}_{1}^{-1}\left(\hat{t}_{s}-t\right)$; (b) a zoom-in view of (a) in the extrapolation interval; (c) maximum vorticity $\|\omega\|_{\infty}$ and its inverse power-law fit $\hat{c}\left(\hat{t}_{s}-t\right)^{-\hat{\gamma}_{2}}$; (d) a zoom-in view of (c) in the extrapolation interval. All results shown in this figure are computed on the $2048 \times 2048$ mesh.

4.4.4. A comparison. We conclude this section with a brief comparison of our results with other representative numerical studies (Table 15). As is clear from the table, our computation offers a much higher effective mesh resolution and advances the solution to a point that is asymptotically closer to the predicted singularity time. It also produces a much stronger vorticity amplification. In short, our computation gives much more convincing evidence for the existence of a finite-time singularity compared with other numerical studies. 
TABLE 15

Comparison of our results with other representative numerical studies. $\tau_{2}$ : the last time at which the solution is deemed "well resolved."

\begin{tabular}{ccccc}
\hline Studies & $\tau_{2}$ & $t_{s}$ & Effec. res. & Vort. amp. \\
\hline$[38]$ & 17 & 18.7 & $\leq 512^{3}$ & 23 \\
{$[5]$} & $1.6^{\dagger}$ & 2.06 & $1024^{3}$ & 180 \\
{$[26]$} & 1.32 & 1.355 & $2048^{3}$ & 21 \\
{$[43]$} & 2.72 & 2.75 & $1024^{3}$ & 55 \\
Ours & 0.003505 & 0.0035056 & $\left(3 \times 10^{12}\right)^{2}$ & $3 \times 10^{8}$ \\
\hline$\dagger:$ According to $[33]$.
\end{tabular}

TABLE 16

The line fitting (4.7) of the $2 m$ th vorticity moment $\Omega_{2 m}, m=2,3,4$, computed on the interval $\left[\tau_{1}, \tau_{2}\right]$. For comparison, the singularity time $\hat{t}_{s}$ estimated from (4.4) is also included.

\begin{tabular}{cccccccc}
\hline \multirow{2}{*}{ Mesh size } & \multirow{2}{*}{$\hat{t}_{s}$ from (4.4) } & \multicolumn{3}{c}{$\hat{t}_{2 m, s}$} & \multicolumn{3}{c}{$\hat{\gamma}_{2 m, 1}$} \\
\cline { 3 - 8 } & & $m=2$ & $m=3$ & $m=4$ & $m=2$ & $m=3$ & $m=4$ \\
\hline $1024 \times 1024$ & 0.0035070 & 0.0035231 & 0.0035124 & 0.0035097 & 1.2542 & 1.6129 & 1.8176 \\
$1280 \times 1280$ & 0.0035063 & 0.0035115 & 0.0035074 & 0.0035067 & 1.1306 & 1.5383 & 1.7658 \\
$1536 \times 1536$ & 0.0035056 & 0.0035056 & 0.0035056 & 0.0035056 & 1.0019 & 1.4857 & 1.7289 \\
$1792 \times 1792$ & 0.0035056 & 0.0035057 & 0.0035056 & 0.0035056 & 1.0039 & 1.4855 & 1.7285 \\
$2048 \times 2048$ & 0.0035056 & 0.0035057 & 0.0035056 & 0.0035056 & 1.0062 & 1.4857 & 1.7285 \\
\hline
\end{tabular}

4.5. Asymptotic scaling analysis II: Vorticity moments. Given the existence of a finite-time singularity as indicated by the blowing-up maximum vorticity $\|\omega\|_{\infty}$, we turn to the interesting question of whether the vorticity moment integrals

$$
\Omega_{2 m}=\left(\int_{D(1, L)}|\omega|^{2 m} d x\right)^{1 / 2 m}, \quad m=1,2, \ldots,
$$

blow up at the same time as $\|\omega\|_{\infty}$ does, and if yes, what type of asymptotic scalings they satisfy. According to Hölder's inequality, higher vorticity moments "control" the growth of lower vorticity moments in the sense that

$$
\Omega_{2 m} \leq \Omega_{2 n}|D(1, L)|^{(n-m) /(2 m n)}, \quad 1 \leq m<n .
$$

Thus the blowup of any vorticity moment $\Omega_{2 m}$ implies the blowup of all higher moments $\Omega_{2 n}(n \geq m)$. In particular, since $\|\omega\|_{\infty}=\Omega_{\infty}$, the blowup of any finite-order vorticity moment implies the blowup of the maximum vorticity, hence providing additional supporting evidence for the existence of a finite-time singularity.

We have carried out a detailed analysis of the vorticity moments and discovered that all moments of order higher than 2 blow up at a finite time. ${ }^{8}$ For the purpose of illustration, we report in Table 16 the singularity time $\hat{t}_{2 m, s}$ and the scaling exponent $\hat{\gamma}_{2 m, 1}$ estimated from the line fitting

$$
y(t):=\left[\frac{d}{d t} \log \Omega_{2 m}(t)\right]^{-1} \sim-\frac{1}{\gamma_{2 m}}\left(t-t_{s}\right)=: a t+b
$$

for $m=2,3,4$, where $\Omega_{2 m}$ is assumed to satisfy the scaling law

$$
\Omega_{2 m}(t) \sim c_{2 m}\left(t_{s}-t\right)^{-\gamma_{2 m}}, \quad c_{2 m}, \gamma_{2 m}>0 .
$$

\footnotetext{
${ }^{8}$ The enstrophy integral, $\Omega_{2}^{2}$, is observed to grow rapidly (faster than double-exponential), but careful analysis indicates that it is likely to remain bounded as the singularity time $t_{s}$ is approached.
} 
It can be observed from this table that all $\Omega_{2 m}$ with $m>1$ satisfy an inverse powerlaw with an exponent monotonically approaching $\hat{\gamma} \approx \frac{5}{2}$, and they all blow up at a finite time $\hat{t}_{2 m, s}$ approximately equal to the singularity time $\hat{t}_{s}$ estimated from (4.4). This confirms the blowup of $\|\omega\|_{\infty}$ at the predicted singularity time $t_{s}$ and hence the existence of a finite-time singularity.

4.6. Vorticity direction and spectral dynamics. The BKM criterion characterizes the finite-time blowup of the 3D Euler equations in terms of the maximum vorticity magnitude $|\omega|$ but makes no assumption on the vorticity direction $\xi=\omega /|\omega|$. It turns out that when less regularity is required on the vorticity magnitude, say boundedness in $L^{p}(p<\infty)$ instead of boundedness in $L^{\infty}$, the regularity of the vorticity direction can also play a role in controlling the blowup of the Euler solutions [17]. To see more precisely how the direction vector $\xi$ enters the analysis, recall the vorticity amplification equation

$$
|\omega|_{t}+u \cdot \nabla|\omega|=\alpha|\omega|,
$$

where $\alpha$ is the vorticity amplification factor:

$$
\alpha=\xi \cdot \nabla u \cdot \xi=\xi \cdot S \xi, \quad S=\frac{1}{2}\left(\nabla u+\nabla u^{T}\right) .
$$

It can be shown that $[17]$

$$
\alpha(x)=\frac{3}{4 \pi} \mathrm{P} \cdot \mathrm{V} \cdot \int_{\mathbb{R}^{3}} D(\hat{y}, \xi(x+y), \xi(x))|\omega(x+y)| \frac{d y}{|y|^{3}},
$$

where $\hat{y}=y /|y|$ is the unit vector pointing in the direction of $y$ and

$$
D\left(e_{1}, e_{2}, e_{3}\right)=\left(e_{1} \cdot e_{3}\right) \operatorname{det}\left(e_{1}, e_{2}, e_{3}\right) .
$$

Note that the quantity $D\left(e_{1}, e_{2}, e_{3}\right)$ is small when $e_{2}$ and $e_{3}$ are nearly aligned or antialigned, so a smoothly varying vorticity direction field $\xi$ near a spatial point $x$ can induce strong cancellation in the vorticity amplification $\alpha(x)$, thus preventing the vorticity $|\omega(x)|$ at $x$ from growing unboundedly. The most well known non-blowup criteria in this direction are those of Constantin, Fefferman, and Majda [19] and Deng, $\mathrm{Hou}$, and $\mathrm{Yu}[20]$. Under the assumption that the vorticity direction $\xi$ is "not too twisted" near the location of the maximum vorticity, they show that a suitable upper bound can be obtained for $\alpha$ and hence for $\|\omega\|_{\infty}$, establishing the regularity of the solutions to the 3D Euler equations.

The Constantin-Fefferman-Majda (CFM) and Deng-Hou-Yu (DHY) non-blowup criteria are useful for excluding false blowup candidates, but they cannot be used directly to verify a finite-time singularity. The reason is that these criteria provide only upper bounds for the amplification factor $\alpha$ while a blowup estimate requires a lower bound. Nevertheless, a careful examination of our numerical data against these criteria provides additional evidence for the existence of a finite-time singularity. It also offers additional insights into the nature of the blowup.

In what follows, we shall state the CFM and DHY non-blowup criteria and apply them to our numerical data (sections 4.6.1-4.6.3). We shall also investigate the vorticity amplification factor $\alpha$ directly at the location of the maximum vorticity and establish a connection between $\alpha$ and the eigenstructure of the symmetric strain tensor $S$ (section 4.6.4). Before proceeding, however, we shall point out that the representation formula (4.8c) for the vorticity amplification factor $\alpha$ is valid only in free space 
$\mathbb{R}^{3}$ and does not hold true for periodic-axisymmetric flows bounded by solid walls. In principle, formulas similar to (4.8c) can be derived in bounded and/or periodic domains; for example, in our case the vorticity amplification equation at the location of the maximum vorticity can be shown to take the form (see (4.5b))

$$
\frac{d}{d t}\|\omega(\cdot, t)\|_{\infty}=-\tilde{\psi}_{1, r z}\|\omega(\cdot, t)\|_{\infty}
$$

where

$$
\tilde{\psi}_{1, r z}=\psi_{1, r z}\left(\tilde{q}_{0}\right)=\frac{1}{L} \int_{0}^{1} r^{3} \int_{0}^{L} \omega_{1}(r, z) G_{1, z}(r, z) d z d r
$$

and $G_{1}$ is a certain "fundamental solution" of the five-dimensional Laplace operator. On the other hand, these representation formulas are often considerably more complicated than (4.8c), and in the presence of axial symmetry they may even obscure the connection between the vorticity amplification factor $\alpha$ and the vorticity direction $\xi$, as the formula (4.9) shows. Hence, instead of deriving and using a formula of the form (4.9), we shall apply in what follows the elegant formula (4.8c) directly to our numerical data. Although the analysis that results is not strictly rigorous, it reveals more clearly the role played by the vorticity direction $\xi$, hence leading to a better understanding of the interplay between the geometry of $\xi$ and the dynamics of the vorticity amplification $\alpha$.

4.6.1. The Constantin-Fefferman-Majda criterion. The CFM criterion consists of two parts. To state the results, we first recall the notion of smoothly directed and regularly directed sets.

Let $u=u(x, t)$ be the velocity field for the 3D incompressible Euler equations (1.1) and $X(q, t)$ be the corresponding flow map, defined by

$$
\frac{d X}{d t}=u(X, t), \quad X(q, 0)=q .
$$

Denote by $W_{t}=X\left(W_{0}, t\right)$ the image of a set $W_{0}$ at time $t$ and by $B_{r}(W)$ the neighborhood of $W$ formed with points situated at Euclidean distance not larger than $r$ from $W$. A set $W_{0}$ is said to be smoothly directed if there exist $\rho>0$ and $r \in\left(0, \frac{1}{2} \rho\right]$ such that the following three conditions are satisfied: first, for every $q \in W_{0}^{*}$ where

$$
W_{0}^{*}=\left\{q \in W_{0}:\left|\omega_{0}(q)\right| \neq 0\right\}
$$

and for all $t \in[0, T)$, the vorticity direction $\xi=\omega /|\omega|$ has a Lipschitz extension to the Euclidean ball of radius $4 \rho$ centered at $X(q, t)$ and

$$
M=\lim _{t \rightarrow T} \sup _{q \in W_{0}^{*}} \int_{0}^{t}\|\nabla \xi(\cdot, \tau)\|_{L^{\infty}\left(B_{4 \rho}(X(q, \tau))\right)}^{2} d \tau<\infty ;
$$

second,

$$
\sup _{B_{3 r}\left(W_{t}\right)}|\omega(x, t)| \leq m \sup _{B_{r}\left(W_{t}\right)}|\omega(x, t)|
$$

holds for all $t \in[0, T)$ with $m \geq 0$ constant; and finally,

$$
\sup _{B_{4 \rho}\left(W_{t}\right)}|u(x, t)| \leq U
$$

Copyright $@$ by SIAM. Unauthorized reproduction of this article is prohibited. 
holds for all $t \in[0, T)$. A set $W_{0}$ is said to be regularly directed if there exists $\rho>0$ such that

$$
\sup _{q \in W_{0}^{*}} \int_{0}^{T} K_{\rho}(X(q, t), t) d t<\infty
$$

where

$$
K_{\rho}(x, t)=\int_{|y| \leq \rho}|D(\hat{y}, \xi(x+y, t), \xi(x, t))| \cdot|\omega(x+y, t)| \frac{d y}{|y|^{3}}
$$

and

$$
D(\hat{y}, \xi(x+y), \xi(x))=(\hat{y} \cdot \xi(x)) \operatorname{det}(\hat{y}, \xi(x+y), \xi(x)) .
$$

The CFM criterion asserts the following [19].

Theorem 4.1. Assume $W_{0}$ is smoothly directed. Then there exist $\tau>0$ and $\Gamma>0$ such that

$$
\sup _{B_{r}\left(W_{t}\right)}|\omega(x, t)| \leq \Gamma \sup _{B_{\rho}\left(W_{t_{0}}\right)}\left|\omega\left(x, t_{0}\right)\right|
$$

holds for any $0 \leq t_{0}<T$ and $0 \leq t-t_{0} \leq \tau$.

TheOREM 4.2. Assume $W_{0}$ is regularly directed. Then there exists $\Gamma>0$ such that

$$
\sup _{q \in W_{0}}|\omega(X(q, t), t)| \leq \Gamma \sup _{q \in W_{0}}\left|\omega_{0}(q)\right|
$$

holds for all $t \in[0, T]$.

Both Theorems 4.1 and 4.2 can be reformulated in cylindrical coordinates. To fix the notation in the rest of this section, we shall denote by $x=\left(x_{1}, x_{2}, x_{3}\right)^{T}$ a point in $\mathbb{R}^{3}$ and by $\tilde{x}=\left(r, x_{3}\right)^{T}$ its projection onto the $r z$-plane, where $r=\sqrt{x_{1}^{2}+x_{2}^{2}}$. For any radially symmetric function $f$, we shall write $f(x)$ and $f(\tilde{x})$ interchangeably depending on the context. The notation $B_{\rho}(q)$ can denote a 3D Euclidean ball if its center $q$ is a point in $\mathbb{R}^{3}$ or a $2 \mathrm{D}$ Euclidean ball if $q$ is a point in the $2 \mathrm{D} r z$-plane.

To check our numerical data against the CFM criterion, we define, for each fixed time instant $t$, the neighborhood of the maximum vorticity:

$$
D_{\infty}(t)=\left\{(r, z) \in D\left(1, \frac{1}{4} L\right):|\omega(r, z, t)| \geq \frac{1}{2}\|\omega(\cdot, t)\|_{\infty}\right\} .
$$

As will be demonstrated below in section 4.7 , the diameter of $D_{\infty}(t)$ shrinks rapidly to 0 as the predicted singularity time $t_{s}$ is approached (see Figure 13(a)). Since the maximum vorticity is always attained at $\tilde{q}_{0}=(1,0)^{T}$, i.e., $\tilde{q}_{0} \in D_{\infty}(t)$ for all $t$, it follows that

$$
D_{\infty}(t) \subseteq B_{\delta}\left(\tilde{q}_{0}\right):=\left\{(r, z):(r-1)^{2}+z^{2}<\delta^{2}\right\}
$$

for any fixed $\delta>0$, provided that $t$ is sufficiently close to $t_{s}$. On the other hand, $\tilde{q}_{0}$ is a stagnation point of the flow field,

$$
\begin{gathered}
u^{r}\left(\tilde{q}_{0}\right)=-\psi_{1, z}\left(\tilde{q}_{0}\right)=0, \quad u^{\theta}\left(\tilde{q}_{0}\right)=u_{1}\left(\tilde{q}_{0}\right)=0, \\
u^{z}\left(\tilde{q}_{0}\right)=2 \psi_{1}\left(\tilde{q}_{0}\right)+\psi_{1, r}\left(\tilde{q}_{0}\right)=0,
\end{gathered}
$$

Copyright (c) by SIAM. Unauthorized reproduction of this article is prohibited. 
in view of the no-flow boundary condition $\psi_{1}(1, z)=0$ (see (2.2c)) and the odd symmetry of $u_{1}, \psi_{1}$ at $z=0$ (see section 2 ). This means that

$$
X\left(q_{0}, t\right) \equiv q_{0}, \quad q_{0}=(1,0,0)^{T} \forall t>0,
$$

and thus for any fixed $\rho>0$ and $t$ sufficiently close to $t_{s}$, the projection of the 3D Euclidean ball $B_{4 \rho}\left(X\left(q_{0}, t\right)\right) \equiv B_{4 \rho}\left(q_{0}\right)$ onto the $r z$-plane will always contain the set $D_{\infty}(t)$.

We are now ready to show that Theorem 4.1, when applied to our numerical data, does not exclude the possibility of a finite-time singularity. More specifically, we shall show that the condition (4.10a) that is required to define a smoothly directed set is not met by our numerical data. For this purpose, we take

$$
W_{0}=W_{0}^{*}=\left\{\left(x_{1}, x_{2}, x_{3}\right) \in \mathbb{R}^{3}:\left(\sqrt{x_{1}^{2}+x_{2}^{2}}, x_{3}\right) \in D_{\infty}(0)\right\}
$$

and note that

$$
\begin{aligned}
\sup _{q \in W_{0}^{*}} \int_{0}^{t} \| & \nabla \xi(\cdot, \tau) \|_{L^{\infty}\left(B_{4 \rho}(X(q, \tau))\right)}^{2} d \tau \\
& \geq \int_{t_{0}}^{t}\|\nabla \xi(\cdot, \tau)\|_{L^{\infty}\left(B_{4 \rho}\left(q_{0}\right)\right)}^{2} d \tau \geq \int_{t_{0}}^{t}\|\nabla \xi(\cdot, \tau)\|_{L^{\infty}\left(D_{\infty}(\tau)\right)}^{2} d \tau
\end{aligned}
$$

for any $t_{0} \in\left(0, t_{s}\right)$ sufficiently close to $t_{s}$ and any $t \in\left(t_{0}, t_{s}\right)$. This shows that, with $T=t_{s}$,

$$
M \geq \lim _{t \rightarrow t_{s}} \int_{t_{0}}^{t}\|\nabla \xi(\cdot, \tau)\|_{L^{\infty}\left(D_{\infty}(\tau)\right)}^{2} d \tau .
$$

To obtain a lower bound for the above integral, we consider the quantity

$$
L_{\xi, \tilde{q}_{0}}(t)=\sup _{\tilde{y} \in D_{\infty}(t)} \frac{\left|\xi(\tilde{y}, t)-\xi\left(\tilde{q}_{0}, t\right)\right|}{\left|\tilde{y}-\tilde{q}_{0}\right|},
$$

which defines the (local) Lipschitz constant of the vorticity direction $\xi$ at $\tilde{q}_{0}$ and which gives a lower bound of $\|\nabla \xi\|_{L^{\infty}\left(D_{\infty}(\tau)\right)}$ in view of the standard estimate

$$
\begin{aligned}
\left|\xi(\tilde{y}, t)-\xi\left(\tilde{q}_{0}, t\right)\right| & \leq \int_{0}^{1}\left|\nabla \xi\left(\tilde{q}_{0}+s\left(\tilde{y}-\tilde{q}_{0}\right), t\right)\right| \cdot\left|\tilde{y}-\tilde{q}_{0}\right| d s \\
& \leq\|\nabla \xi(\cdot, t)\|_{L^{\infty}\left(D_{\infty}(t)\right)}\left|\tilde{y}-\tilde{q}_{0}\right|
\end{aligned}
$$

(we note that $D_{\infty}(t)$ is convex; see Figure $13(\mathrm{c})$ ). Since the quantity $L_{\xi, \tilde{q}_{0}}$ estimated from our numerical data grows rapidly with $t$, as is clear from Figure 10, and a line fitting similar to (4.6) yields

$$
L_{\xi, \tilde{q}_{0}}(t) \sim c\left(\hat{t}_{s}-t\right)^{-2.9165}, \quad c=1.3497 \times 10^{-7},
$$

where $\hat{t}_{s}$ is the singularity time estimated from (4.4), it follows that the time integral of $\|\nabla \xi\|_{L^{\infty}\left(D_{\infty}(\tau)\right)}^{2}$ cannot remain bounded as $t$ approaches $t_{s}$. Hence (4.10a) cannot be satisfied by our choice of $W_{0}$. Returning to the statement of Theorem 4.1, we see that

$$
\sup _{B_{r}\left(W_{t}\right)}|\omega(x, t)|=\|\omega(\cdot, t)\|_{\infty}
$$

Copyright (C) by SIAM. Unauthorized reproduction of this article is prohibited. 


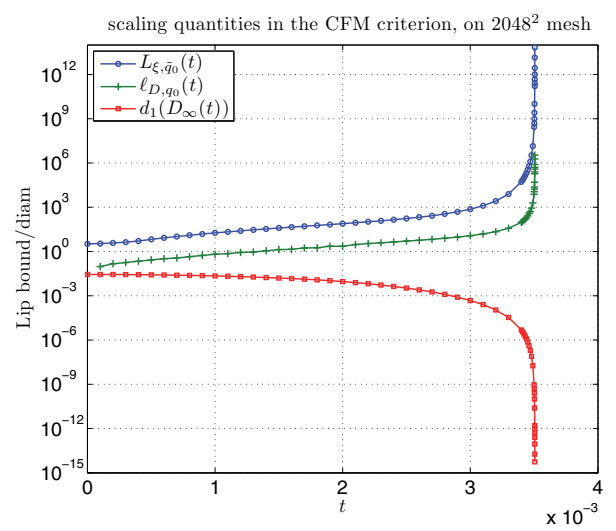

FIG. 10. The local Lipschitz constants $L_{\xi, \tilde{q}_{0}}, \ell_{D, q_{0}}$ and the length scale $d_{1}$ considered in the CFM criterion.

since $q_{0}$, the location of the maximum vorticity, lies in $W_{t}$ for all $t$. This shows that no a priori bound on the maximum vorticity can be inferred from Theorem 4.1.

Similarly, we can argue that Theorem 4.2, when applied to our numerical data, does not exclude the possibility of a finite-time singularity. To see this, we choose $W_{0}$ as above and note that

$$
\sup _{q \in W_{0}^{*}} \int_{0}^{t_{s}} K_{\rho}(X(q, t), t) d t \geq \int_{0}^{t_{s}} K_{\rho}\left(q_{0}, t\right) d t
$$

where

$$
K_{\rho}\left(q_{0}, t\right)=\int_{|y| \leq \rho}\left|D\left(\hat{y}, \xi\left(q_{0}+y, t\right), \xi\left(q_{0}, t\right)\right)\right| \cdot\left|\omega\left(q_{0}+y, t\right)\right| \frac{d y}{|y|^{3}} .
$$

The above integral has a lower bound estimate (Appendix D)

$$
K_{\rho}\left(q_{0}, t\right) \geq \frac{3 \pi}{640} d_{1}\left(D_{\infty}(t)\right)\|\omega(\cdot, t)\|_{\infty} \ell_{D, q_{0}}(t)
$$

where $\ell_{D, q_{0}}$ is the infimum of $|D| /|y|$ over some neighborhood of $q_{0}$ and $d_{1}$ is (roughly) the diameter of $D_{\infty}(t)$. Thus to complete the analysis, it suffices to estimate the quantities $\|\omega\|_{\infty}, d_{1}$, and $\ell_{D, q_{0}}$ from the numerical data. The estimate of $\|\omega\|_{\infty}$ is derived in section 4.4.3 and has the form

$$
\|\omega(\cdot, t)\|_{\infty} \sim c_{1}\left(t_{s}-t\right)^{-2.4568}, \quad c_{1}=7.3273 \times 10^{-4} .
$$

As for the other two quantities, it is observed that $\ell_{D, q_{0}}$ grows rapidly with $t$ while $d_{1}$ decays with $t$ (Figure 10). A line fitting similar to (4.6) then yields

$$
\begin{aligned}
\ell_{D, q_{0}}(t) \sim c_{2}\left(\hat{t}_{s}-t\right)^{-1.4597}, & c_{2}=1.7596 \times 10^{-4}, \\
d_{1}\left(D_{\infty}(t)\right) \sim\left[\delta^{-1}\left(\hat{t}_{s}-t\right)\right]^{2.9181}, & \delta=7.0214 \times 10^{-3},
\end{aligned}
$$

which, together with the estimate of $\|\omega\|_{\infty}$, shows that

$$
K_{\rho}\left(q_{0}, t\right) \geq C\left(t_{s}-t\right)^{-0.9984} .
$$


Taking into account the effect of numerical errors, we may conclude that $K_{\rho}\left(q_{0}, t\right) \gtrsim$ $C\left(t_{s}-t\right)^{-1}$ and the time integral of $K_{\rho}\left(q_{0}, t\right)$ diverges as $t$ approaches $t_{s}$. Thus the condition (4.11a) is not satisfied by our numerical data.

At first glance, the estimate (4.13b) may look a bit surprising, because the growth of the maximum vorticity $\|\omega\|_{\infty}$ is so strong while the blowup of $K_{\rho}\left(q_{0}\right)$ implied from (4.13b) is so marginal. Still, we believe this is not unreasonable, because (4.13b) provides only a lower bound for $K_{\rho}\left(q_{0}\right)$ which does not necessarily capture the rapid growth of $K_{\rho}\left(q_{0}\right)$. More importantly, both $K_{\rho}\left(q_{0}\right)$ and the amplification factor $\alpha\left(q_{0}\right)$ are roughly of the same order if $D\left(\hat{y}, \xi\left(q_{0}+y\right), \xi\left(q_{0}\right)\right)$ does not change sign in a neighborhood of $y=0$ (see (4.8c)). Since $\alpha\left(q_{0}\right)$ must grow like $\left(t_{s}-t\right)^{-1}$ if the maximum vorticity obeys an inverse power-law, the "marginal blowup" of $K_{\rho}\left(q_{0}\right)$ as indicated by (4.13b) may indeed be what is to be expected.

We also emphasize that the above analysis is purely formal, since the representation formula (4.11b) for the quantity $K_{\rho}(x)$ is not valid in bounded and/or periodic domains. On the other hand, the analysis suggests, through the key estimate (4.13a), that the formation of a singularity in the $3 \mathrm{D}$ Euler equations is likely a result of the subtle balance among the three competing "forces," namely the growth rate of the maximum vorticity $\|\omega\|_{\infty}$, the collapsing rate of the support of the vorticity as measured by $d_{1}$, and the smoothness of the vorticity direction field as measured by $\ell_{D, q_{0}}$. This observation is expected to hold true even in bounded and/or periodic domains where $(4.11 \mathrm{~b})$ is not valid, and this is where the significance of the above formal analysis lies.

4.6.2. The Deng-Hou-Yu criterion. The DHY criterion improves the nonblowup criterion of CFM, in particular the part stated in Theorem 4.1, by relaxing the regularity assumptions made on the velocity field $u$ and the vorticity direction $\xi$. Instead of assuming the integrability of the gradient of $\xi$ in an $O(1)$ region, the DHY criterion requires only the integrability of the divergence of $\xi$ along a vortex line segment whose length is allowed to shrink to 0 (Theorem 4.3). In addition, the velocity field $u$ is allowed to grow unboundedly in time, provided that a mild partial regularity condition on $u$ is satisfied along a vortex line (Theorem 4.4). These improvements make the criterion easier to apply in actual numerical simulations.

Like the CFM criterion, the DHY criterion consists of two parts, the first of which excludes the possibility of a point singularity under a certain regularity assumption on the divergence of the vorticity direction, $\nabla \cdot \xi$.

THEOREM 4.3. Consider the $3 D$ incompressible Euler equations (1.1), and let $x(t)$ be a family of points such that

$$
|\omega(x(t), t)| \geq c_{0}\|\omega(\cdot, t)\|_{\infty}
$$

for some absolute constant $c_{0}>0$. Let $y(t)$ be another family of points such that, for each $t \in[0, T), y(t)$ lies on the same vortex line as $x(t)$ and the vorticity direction $\xi=\omega /|\omega|$ is well defined along the vortex line lying between $x(t)$ and $y(t)$. If

$$
\left|\int_{x(t)}^{y(t)}(\nabla \cdot \xi)(s, t) d s\right| \leq C \quad \forall t \in[0, T)
$$

for some absolute constant $C$ and

$$
\int_{0}^{T}|\omega(y(t), t)| d t<\infty
$$

Copyright $@$ ㅇ by SIAM. Unauthorized reproduction of this article is prohibited. 
then there will be no blowup of $\omega(x(t), t)$ up to time T. Moreover,

$$
\mathrm{e}^{-C} \leq \frac{|\omega(x(t), t)|}{|\omega(y(t), t)|} \leq \mathrm{e}^{C} \quad \forall t \in[0, T) .
$$

The second part of the DHY criterion concerns the dynamic blowup of the vorticity along a vortex line. More specifically, consider a family of vortex line segments $L_{t}$ along which the vorticity is comparable to $\|\omega(\cdot, t)\|_{\infty}$. Denote by $L(t)$ the arc length of $L_{t}$, and define

$$
U_{\xi}(t)=\max _{x, y \in L_{t}}|(u \cdot \xi)(x, t)-(u \cdot \xi)(y, t)|, \quad U_{n}(t)=\max _{L_{t}}|u \cdot n|,
$$

and

$$
M(t)=\max \left\{\|\nabla \cdot \xi\|_{L^{\infty}\left(L_{t}\right)},\|\kappa\|_{L^{\infty}\left(L_{t}\right)}\right\},
$$

where $\kappa=|\xi \cdot \nabla \xi|$ is the curvature of the vortex line and $n$ is the unit normal vector of $L_{t}$.

TheOREM 4.4. Assume that there exist a family of vortex line segments $L_{t}$ and a $T_{0} \in[0, T)$ such that $X\left(L_{t_{1}}, t_{1}, t_{2}\right) \supseteq L_{t_{2}}$ for all $T_{0}<t_{1}<t_{2}<T$. Assume also that $\|\omega(\cdot, t)\|_{\infty}$ is monotonically increasing and that

$$
\|\omega(\cdot, t)\|_{L^{\infty}\left(L_{t}\right)} \geq c_{0}\|\omega(\cdot, t)\|_{\infty}
$$

for some absolute constant $c_{0}>0$ when $t$ is sufficiently close to $T$. If

(i) $U_{\xi}(t)+U_{n}(t) M(t) L(t) \leq c_{A}(T-t)^{-A}$ for some $A \in(0,1)$,

(ii) $M(t) L(t) \leq C_{0}$, and

(iii) $L(t) \geq c_{B}(T-t)^{B}$ for some $B<1-A$,

where $c_{A}, c_{B}, C_{0}$ are all absolute constants, then there will be no blowup of $\|\omega(\cdot, t)\|_{\infty}$ up to time $T$.

To check our numerical data against the DHY criterion, we first note that any vortex line segment containing the point $q_{0}=(1,0,0)^{T}$ must lie on the ray

$$
\left[0, q_{0}\right]:=\left\{\left(x_{1}, 0,0\right) \in \mathbb{R}^{3}: x_{1} \in(0,1)\right\} .
$$

This follows directly from the fact that the vorticity direction vectors $\xi(x)$, when restricted to $\left[0, q_{0}\right]$, all point in the same direction $(-1,0,0)^{T}$. Now we argue that the conditions of Theorem 4.3 cannot be satisfied for the particular choice $x(t) \equiv q_{0}$. Indeed, if $y(t)$ is a family of points satisfying the conditions of the theorem, then each $y(t)$ must lie on the same vortex line as $q_{0}$ and hence must lie on the ray $\left[0, q_{0}\right]$. Now assume $y(t)$ is the image of a Lagrangian marker ${ }^{9}$ and consider the quantity

$$
i_{1}(t)=\min _{x \in\left[0, q_{0}\right]}\left\{\int_{0}^{t}|\omega(x, s)| d s+\int_{x}^{q_{0}}(\nabla \cdot \xi)(y, t) d y\right\} .
$$

If we define, for each fixed $t \in\left(0, t_{s}\right)$ and $q \in\left(0, q_{0}\right)$, the particle trajectory

$$
\frac{d X_{q}}{d s}=u^{r}\left(X_{q}, s\right)=-\left|X_{q}\right| \psi_{1, z}\left(X_{q}, s\right), \quad X_{q}(t)=q
$$

\footnotetext{
${ }^{9}$ The same analysis applies to more general $y(t)$ after suitable modifications, but for simplicity we shall confine ourselves to the special case $y(t)=X(q, t)$, i.e., $y(t)$ is a Lagrangian marker.
}

Copyright $@$ by SIAM. Unauthorized reproduction of this article is prohibited. 


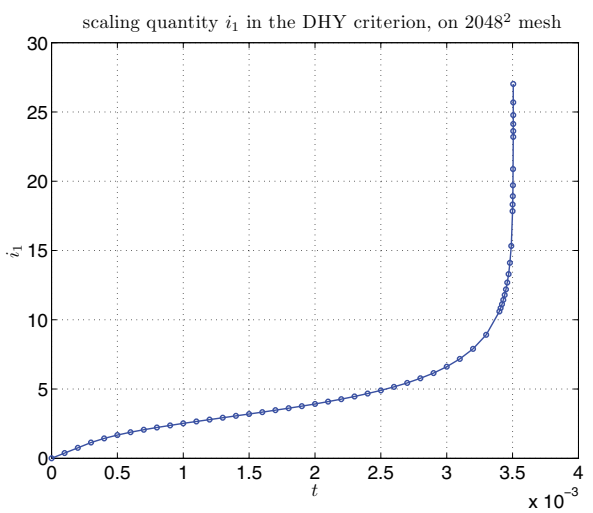

(a) $i_{1}(t)$

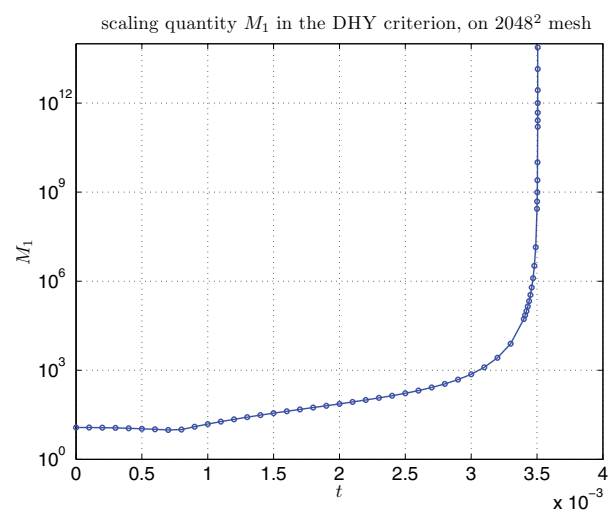

(b) $M_{1}(t)$

FIG. 11. The quantities $i_{1}$ and $M_{1}$ considered in the DHY criterion.

then clearly $i_{1}$ gives a lower bound for

$$
\int_{0}^{t}\left|\omega\left(X_{q}(s), s\right)\right| d s+\left|\int_{q}^{q_{0}}(\nabla \cdot \xi)(y, t) d y\right|
$$

since it is numerically observed that $u^{r}<0$ on $\left[0, q_{0}\right]$ and $|\omega|$ is monotonically increasing on $\left[0, q_{0}\right]$, which means that

$$
\left|\omega\left(X_{q}(s), s\right)\right| \geq\left|\omega\left(X_{q}(t), s\right)\right|=|\omega(q, s)| \quad \forall s \in[0, t] .
$$

As is clear from Figure 11(a), the quantity $i_{1}$ grows unboundedly as $t$ approaches $t_{s}$, and hence the two conditions (4.14a) and (4.14b) stated in Theorem 4.3 cannot be satisfied simultaneously.

To apply Theorem 4.4 to our data, we consider the quantity

$$
M_{1}(t)=\max _{x \in\left[0, q_{0}\right]}(\nabla \cdot \xi)(x, t),
$$

which defines the local maximum of the divergence of $\xi$ on $\left[0, q_{0}\right]$. As can be seen from Figure 11(b), the quantity $M_{1}(t)$ grows rapidly with $t$, and a line fitting similar to (4.6) shows that

$$
M_{1}(t) \sim c\left(\hat{t}_{s}-t\right)^{-2.9165}, \quad c=1.3497 \times 10^{-7} .
$$

We now argue that the conditions of Theorem 4.4 cannot be satisfied for any family of vortex line segments $L_{t}$ containing the point $q_{0}$. Indeed, as our numerical data shows, the maximum of $\nabla \cdot \xi$ on $\left[0, q_{0}\right]$ is always attained at $q_{0}$, i.e.,

$$
M_{1}(t)=(\nabla \cdot \xi)\left(q_{0}, t\right) \leq\|\nabla \cdot \xi\|_{L^{\infty}\left(L_{t}\right)} \leq M(t) .
$$

Thus conditions (ii) and (iii) in Theorem 4.4 cannot be satisfied simultaneously, since condition (ii), when combined with (4.15), implies that

$$
L(t) \leq C_{0} M^{-1}(t) \leq C_{0} M_{1}^{-1}(t) \sim C\left(\hat{t}_{s}-t\right)^{2.9165},
$$

which violates condition (iii). 


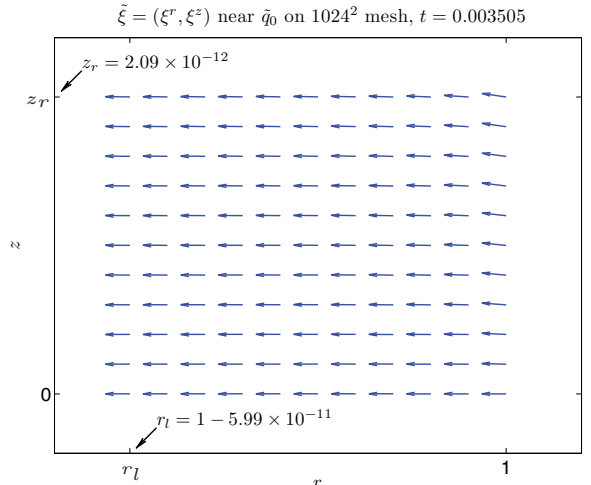

(a) $2 \mathrm{D}$ vorticity direction $\tilde{\xi}=\left(\xi^{r}, \xi^{z}\right)^{T}$

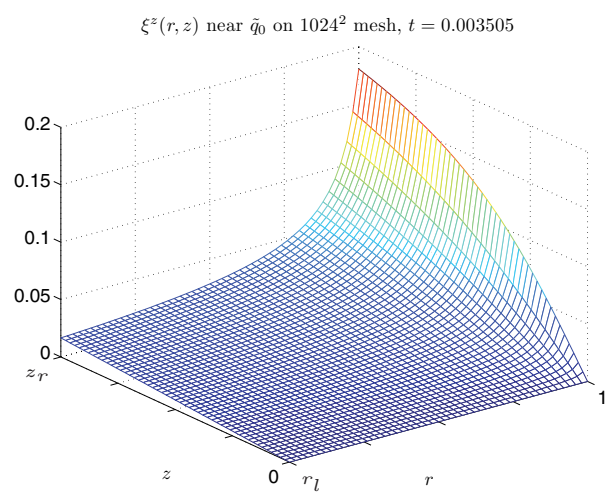

(b) $z$-component $\xi^{z}$ of vorticity direction

FIG. 12. The geometry of the vorticity direction: (a) the $2 D$ vorticity direction $\tilde{\xi}=\left(\xi^{r}, \xi^{z}\right)^{T}$ and (b) the $z$-direction component $\xi^{z}$ computed on the $1024 \times 1024$ mesh at $t=0.003505$. All plots in this figure are defined on the region $\left[r_{l}, 1\right] \times\left[0, z_{r}\right]$ where $r_{l}=1-5.99 \times 10^{-11}$ and $z_{r}=2.09 \times 10^{-12}$.

4.6.3. The geometry of the vorticity direction. It is illuminating to examine at this point the local geometric structure of the vorticity direction $\xi$ near the location of the maximum vorticity. Figure 12 shows a plot of the $2 \mathrm{D}$ vorticity direction $\tilde{\xi}=\left(\xi^{r}, \xi^{z}\right)^{T}$ and a plot of the $z$-direction component $\xi^{z}$, both defined at $t=0.003505$ on the set

$$
\tilde{D}_{\infty}=\left[1-5.99 \times 10^{-11}, 1\right] \times\left[0,2.09 \times 10^{-12}\right] .
$$

The through-plane $(\theta)$ component of $\xi$ has a maximum absolute value of $2.1874 \times 10^{-6}$ in $\tilde{D}_{\infty}$ and hence is negligible there. It can be observed from Figure 12 that the $z$ direction component $\xi^{z}$ experiences an $O(1)$ change in $\tilde{D}_{\infty}$ along the $z$-dimension. This indicates the formation of bundles of "densely packed" vortex lines near the location of the maximum vorticity, which explains the rapid growth of quantities like $L_{\xi, \tilde{q}_{0}}$ and $\nabla \cdot \xi$ observed in Figures $10-11$.

4.6.4. The spectral dynamics. The analysis carried out in the previous sections suggests that the growth of the vorticity amplification factor $\alpha$ depends on the local geometric structures of the vorticity vector. Alternatively, the dynamics of the vorticity amplification can be investigated from an algebraic point of view using the defining relation (see $(4.8 \mathrm{~b})$ )

$$
\alpha=\xi \cdot \nabla u \cdot \xi=\xi \cdot S \xi, \quad S=\frac{1}{2}\left(\nabla u+\nabla u^{T}\right),
$$

where the eigenstructure of the symmetric strain tensor $S$ plays the central role.

In what follows, we shall derive a remarkable connection between the vorticity amplification factor $\alpha$ and the eigenstructure of $S$ at the location of the maximum vorticity. The derivation starts with the representation formula of the velocity vector in cylindrical coordinates:

$$
u=u^{r} e_{r}+u^{\theta} e_{\theta}+u^{z} e_{z}
$$

where the three Cartesian components of $u$ are expressed in terms of the transformed 
variables $\left(u_{1}, \psi_{1}\right)$ :

$$
\begin{aligned}
& v_{1}=-r \psi_{1, z} \cos \theta-r u_{1} \sin \theta, \\
& v_{2}=-r \psi_{1, z} \sin \theta+r u_{1} \cos \theta, \\
& v_{3}=2 \psi_{1}+r \psi_{1, r} .
\end{aligned}
$$

The entries of the deformation tensor $\nabla u$ can be readily computed, yielding

$$
\begin{array}{lll}
\left.\partial_{x} v_{1}\right|_{\theta=0}=-\psi_{1, z}-r \psi_{1, r z}, & \left.\partial_{y} v_{1}\right|_{\theta=0}=-u_{1}, & \left.\partial_{z} v_{1}\right|_{\theta=0}=-r \psi_{1, z z}, \\
\left.\partial_{x} v_{2}\right|_{\theta=0}=u_{1}+r u_{1, r}, & \left.\partial_{y} v_{2}\right|_{\theta=0}=-\psi_{1, z}, & \left.\partial_{z} v_{2}\right|_{\theta=0}=r u_{1, z}, \\
\left.\partial_{x} v_{3}\right|_{\theta=0}=3 \psi_{1, r}+r \psi_{1, r r}, & \left.\partial_{y} v_{3}\right|_{\theta=0}=0, & \left.\partial_{z} v_{3}\right|_{\theta=0}=2 \psi_{1, z}+r \psi_{1, r z} .
\end{array}
$$

Note that the computation needs only be done on the meridian plane $\theta=0$ thanks to the axial symmetry. When further restricted to the point $\tilde{q}_{0}=(1,0)^{T}$, the location of the maximum vorticity, the above expressions reduce to

$$
\nabla \tilde{u}=\left(\begin{array}{ccc}
-\tilde{\psi}_{1, r z} & 0 & 0 \\
0 & 0 & \tilde{u}_{1, z} \\
0 & 0 & \tilde{\psi}_{1, r z}
\end{array}\right), \quad \tilde{S}=\left(\begin{array}{ccc}
-\tilde{\psi}_{1, r z} & 0 & 0 \\
0 & 0 & \frac{1}{2} \tilde{u}_{1, z} \\
0 & \frac{1}{2} \tilde{u}_{1, z} & \psi_{1, r z}
\end{array}\right),
$$

where for simplicity we have written $\nabla \tilde{u}=\nabla u\left(\tilde{q}_{0}\right), \tilde{S}=S\left(\tilde{q}_{0}\right)$, etc.

Now the eigenvalues of $\tilde{S}$ can be easily found to be

$$
\tilde{\lambda}_{1,3}=\frac{1}{2}\left[\tilde{\psi}_{1, r z} \pm\left(\tilde{\psi}_{1, r z}^{2}+\tilde{u}_{1, z}^{2}\right)^{1 / 2}\right], \quad \tilde{\lambda}_{2}=-\tilde{\psi}_{1, r z}
$$

with corresponding eigenvectors

$$
\tilde{w}_{1}=\left(\begin{array}{c}
0 \\
\frac{1}{2} \tilde{u}_{1, z} \\
\tilde{\lambda}_{1}
\end{array}\right), \quad \tilde{w}_{2}=\left(\begin{array}{c}
1 \\
0 \\
0
\end{array}\right), \quad \tilde{w}_{3}=\left(\begin{array}{c}
0 \\
\frac{1}{2} \tilde{u}_{1, z} \\
\tilde{\lambda}_{3}
\end{array}\right) .
$$

On the other hand, the vorticity vector $\omega$ at $\tilde{q}_{0}$ takes the form (see (4.5a))

$$
\tilde{\omega}=\left(\begin{array}{c}
-\tilde{u}_{1, z} \\
0 \\
0
\end{array}\right), \quad \text { with } \quad \tilde{\xi}=\frac{\tilde{\omega}}{|\tilde{\omega}|}=\left(\begin{array}{c}
-1 \\
0 \\
0
\end{array}\right) .
$$

Thus the vorticity direction $\tilde{\xi}$ at the location of the maximum vorticity is perfectly aligned with $\tilde{w}_{2}$, the second eigenvector of $\tilde{S}$. In addition,

$$
\alpha_{\infty}:=\tilde{\alpha}=\tilde{\xi} \cdot \tilde{S} \tilde{\xi}=\tilde{\lambda}_{2}=-\tilde{\psi}_{1, r z},
$$

consistent with the result derived earlier in section 4.4.1 (see (4.5b)).

It is worth noting that, when viewed in $\mathbb{R}^{3}$, the eigenvectors $\left\{\tilde{w}_{1}, \tilde{w}_{2}, \tilde{w}_{3}\right\}$ restricted to the "singularity ring"

$$
C=\left\{(x, y, z) \in \mathbb{R}^{3}: x^{2}+y^{2}=1, z=0\right\}
$$

form an orthogonal frame, with $\tilde{w}_{2}$ pointing in the radial direction and $\tilde{w}_{1}, \tilde{w}_{3}$ pointing in directions tangential to the lateral surface of the cylinder $r=1$.

Copyright $@$ ( ) by SIAM. Unauthorized reproduction of this article is prohibited. 
Finally, by making use of the relations

$$
\alpha_{\infty}=-\tilde{\psi}_{1, r z}, \quad\|\omega\|_{\infty}=|\tilde{\omega}|=\left|\tilde{u}_{1, z}\right|,
$$

we may also express the first and third eigenvalues of $\tilde{S}$ in the form

$$
\tilde{\lambda}_{1,3}=\frac{1}{2}\left[-\alpha_{\infty} \pm\left(\alpha_{\infty}^{2}+\|\omega\|_{\infty}^{2}\right)^{1 / 2}\right]
$$

Since $\alpha_{\infty}$ and $\|\omega\|_{\infty}$ both satisfy an inverse power-law with an exponent roughly equal to -1 (for $\alpha_{\infty}$ ) and $-\frac{5}{2}$ (for $\|\omega\|_{\infty}$ ), it follows that

$$
\tilde{\lambda}_{1,3} \sim \pm \frac{1}{2}\|\omega\|_{\infty}, \quad t \rightarrow t_{s}^{-} .
$$

This is confirmed by a line fitting similar to (4.6), which yields

$$
\begin{array}{rlrl}
\tilde{\lambda}_{1} & \sim c_{1}\left(\hat{t}_{s}-t\right)^{-2.4582}, & & c_{1}=3.6514 \times 10^{-4}, \\
\tilde{\lambda}_{3} & \sim c_{3}\left(\hat{t}_{s}-t\right)^{-2.4576}, & c_{3}=-3.6759 \times 10^{-4},
\end{array}
$$

where $\hat{t}_{s}$ is the singularity time estimated from (4.4).

4.7. Locally self-similar structure. The $3 \mathrm{D}$ Euler equations (1.1) have the well-known scaling property that if $u(x, t)$ is a solution of the equations, then

$$
u_{\lambda}(x, t):=\lambda^{\alpha} u\left(\lambda x, \lambda^{\alpha+1} t\right) \quad \forall \lambda>0, \forall \alpha \in \mathbb{R}
$$

is also a solution. A natural question is then whether the 3D Euler equations have self-similar solutions of the form

$$
u(x, t)=\frac{1}{[T-t]^{\gamma}} U\left(\frac{x-x_{0}}{[T-t]^{\beta}}\right),
$$

where $U$ is a self-similar velocity profile and $\beta, \gamma$ are scaling exponents. By substituting (4.16a) into (1.1), it is easily shown that

$$
\beta=\frac{1}{\alpha+1}, \quad \gamma=\frac{\alpha}{\alpha+1} \quad \forall \alpha \neq-1,
$$

which in particular implies that

$$
\nabla u(x, t)=\frac{1}{T-t} \nabla U\left(\frac{x-x_{0}}{[T-t]^{\beta}}\right) .
$$

In $[11,12]$, the existence of global self-similar solutions of the form $(4.16 \mathrm{c})$ is excluded under one of the following conditions: either [12]

$$
\limsup _{t \rightarrow T^{-}}(T-t)\|\nabla u(\cdot, t)\|_{\infty}=\|\nabla U\|_{\infty}<1,
$$

or $[11]$

$$
\Omega:=\nabla \times U \neq 0 \quad \text { and } \quad \Omega \in L^{p}\left(\mathbb{R}^{3}\right) \quad \forall p \in\left(0, p_{1}\right)
$$

for some $p_{1}>0$. Note that the first condition is not easy to interpret physically while the second is too strong, effectively requiring that $\Omega$ decay exponentially fast at 
infinity or have compact support. These nonexistence results were generalized later in [12] to $\alpha$-asymptotically global self-similar solutions $\bar{U}$ of the form

$$
\lim _{t \rightarrow T^{-}}[T-t]^{1-(3 \beta / p)}\left\|\nabla u(\cdot, t)-\frac{1}{T-t} \nabla \bar{U}\left(\frac{\cdot-x_{0}}{[T-t]^{\beta}}\right)\right\|_{L^{p}\left(\mathbb{R}^{3}\right)}=0,
$$

where $\beta=(\alpha+1)^{-1}$ and $p \in(0, \infty]$. Similar nonexistence results for local self-similar solutions were also obtained in [13].

In axisymmetric flows, self-similar solutions ${ }^{10}$ naturally take the form

$$
\begin{aligned}
& u_{1}(\tilde{x}, t) \sim[T-t]^{\gamma_{u}} U\left(\frac{\tilde{x}-\tilde{x}_{0}}{[T-t]^{\gamma_{l}}}\right), \\
& \omega_{1}(\tilde{x}, t) \sim[T-t]^{\gamma_{\omega}} \Omega\left(\frac{\tilde{x}-\tilde{x}_{0}}{[T-t]^{\gamma_{l}}}\right), \\
& \psi_{1}(\tilde{x}, t) \sim[T-t]^{\gamma_{\psi}} \Psi\left(\frac{\tilde{x}-\tilde{x}_{0}}{[T-t]^{\gamma_{l}}}\right), \quad \tilde{x} \rightarrow \tilde{x}_{0}, t \rightarrow T^{-},
\end{aligned}
$$

where $\tilde{x}=(r, z)^{T}$ is a point on the $r z$-plane and $(U, \Omega, \Psi)$ are self-similar profiles. Note that this ansatz does not correspond to a Leray-type self-similar solution when viewed in $\mathbb{R}^{3}$; rather, it describes a tube-like anisotropic singularity due to the axial symmetry. In addition, the ansatz induces a scaling law (see section 4.7.3)

$$
\|\nabla u(\cdot, t)\|_{\infty}=O(T-t)^{\min \left\{\gamma_{u}-\gamma_{\ell},-1\right\}}
$$

that is very different from the "standard" law $\|\nabla u(\cdot, t)\|_{\infty}=O(T-t)^{-1}$ assumed by $[11,12,13]$. Hence it gives new hope for the existence of a (meridian-plane) self-similar solution.

In what follows, we shall carry out an extensive study of the numerical solution near the location of the maximum vorticity $\tilde{q}_{0}=(1,0)^{T}$ and demonstrate the existence of a locally self-similar blowup. By applying a line fitting similar to (4.6), we also deduce the scaling law $\|\nabla u(\cdot, t)\|_{\infty}=O(T-t)^{-2.4529}$ satisfied by the self-similar solution, confirming again the existence of a finite-time singularity.

4.7.1. Existence of self-similar neighborhood. The identification of a locally self-similar solution requires the identification of its three basic ingredients: first, the center of self-similarity, $\tilde{x}_{0}$, around which the self-similar structure is developed; second, a neighborhood of $\tilde{x}_{0}$ in which the self-similar behavior is observed; third, a self-similar profile based on which the self-similar solution is determined. In our computations, the center of self-similarity must be $\tilde{q}_{0}=(1,0)^{T}$, the location of the maximum vorticity, since this is the point at which the solution is about to blow up. To identify a "self-similar neighborhood" of $\tilde{q}_{0}$, we consider again the set $D_{\infty}(t)$ introduced in (4.12),

$$
D_{\infty}(t)=\left\{(r, z) \in D\left(1, \frac{1}{4} L\right):|\omega(r, z, t)| \geq \frac{1}{2}\|\omega(\cdot, t)\|_{\infty}\right\}
$$

and plot in Figure 13(a) the boundary curves of $D_{\infty}(t)$,

$$
C_{\infty}(t)=\left\{(r, z) \in D\left(1, \frac{1}{4} L\right):|\omega(r, z, t)|=\frac{1}{2}\|\omega(\cdot, t)\|_{\infty}\right\}
$$

\footnotetext{
${ }^{10}$ In what follows, whenever we say "self-similar solutions" for an axisymmetric flow we always mean "self-similar solutions in the meridian plane."
}

Copyright $@$ by SIAM. Unauthorized reproduction of this article is prohibited. 


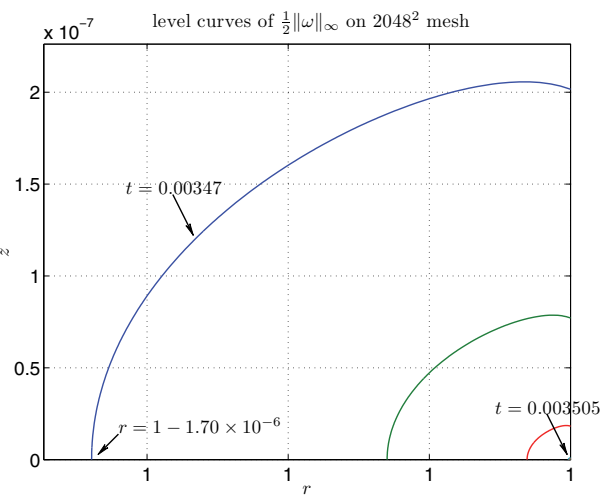

(a) linear-linear

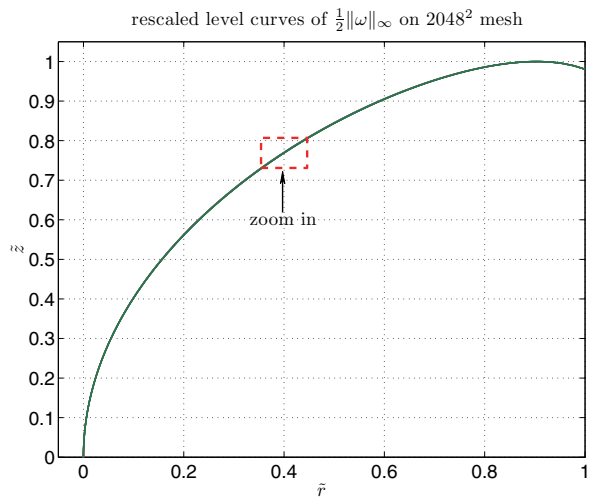

(c) rescaled

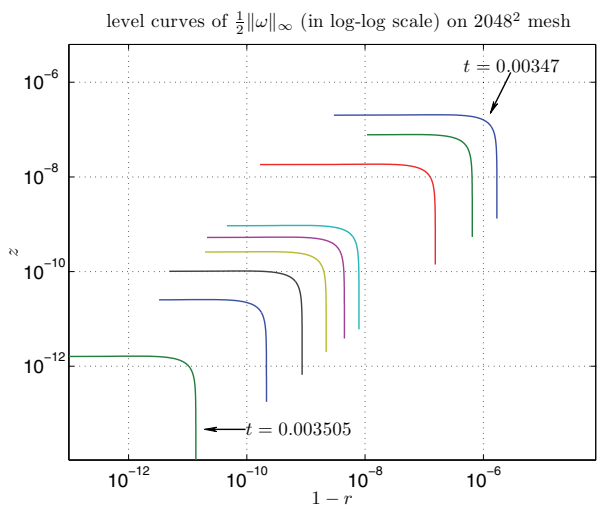

(b) $\log -\log$

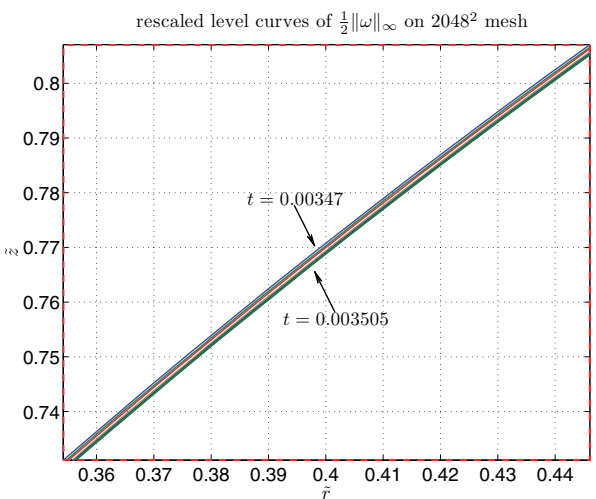

(d) rescaled (zoom-in)

FIG. 13. The level curves $C_{\infty}(t)$ at various time instants in (a) linear-linear and (b) log-log scale (against the variables $(1-r)$ and $z$ ). The rescaled level curves and their zoom-in view are shown in (c) and (d).

at the nine time instants

$$
\begin{aligned}
& \mathcal{T}_{s}=\{0.00347,0.00348,0.00349,0.0035 \\
&0.003501,0.003502,0.003503,0.003504,0.003505\} .
\end{aligned}
$$

It can be observed from the figure that the level curves $C_{\infty}(t)$ shrink rapidly toward $\tilde{q}_{0}$ and have very similar shapes at the first few time instants when they are still visible in the figure. To reveal more clearly the asymptotic behavior of $C_{\infty}(t)$ at the later times, we plot the same nine curves in Figure 13(b) in log-log scale against the variables $(1-r)$ and $z$. The results show that the shape of $C_{\infty}(t)$ indeed remains roughly the same at all nine time instants. Motivated by this observation, we rescale each curve $C_{\infty}(t)$ according to the rule

$$
\tilde{r}=1-\frac{1-r}{d_{r}\left(C_{\infty}(t)\right)}, \quad \tilde{z}=\frac{z}{d_{z}\left(C_{\infty}(t)\right)},
$$

where

$$
d_{r}\left(C_{\infty}(t)\right)=\max _{(r, z) \in C_{\infty}(t)}|1-r|, \quad d_{z}\left(C_{\infty}(t)\right)=\max _{(r, z) \in C_{\infty}(t)}|z| .
$$

Copyright $@$ by SIAM. Unauthorized reproduction of this article is prohibited. 


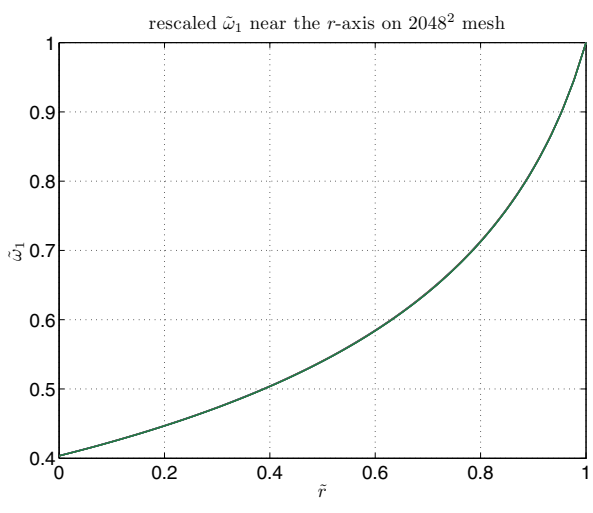

(a) near the $r$-axis

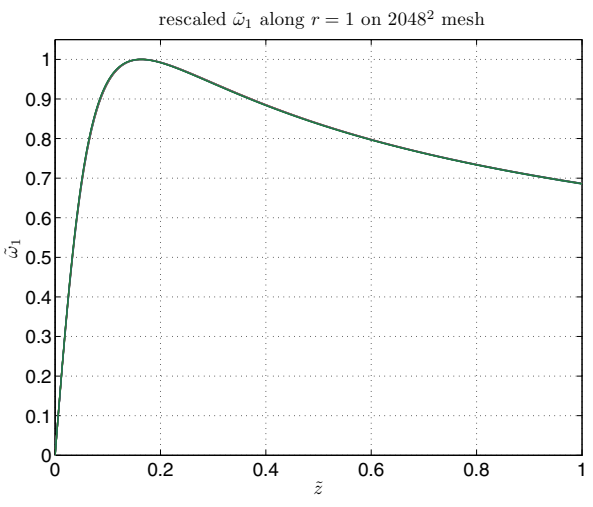

(b) along the wall $r=1$

FIG. 14. The $1 D$ self-similar profiles of $\omega_{1}$ (a) near the $r$-axis and (b) along the wall $r=1$, obtained by rescaling the solutions at the nine time instants given by (4.19).

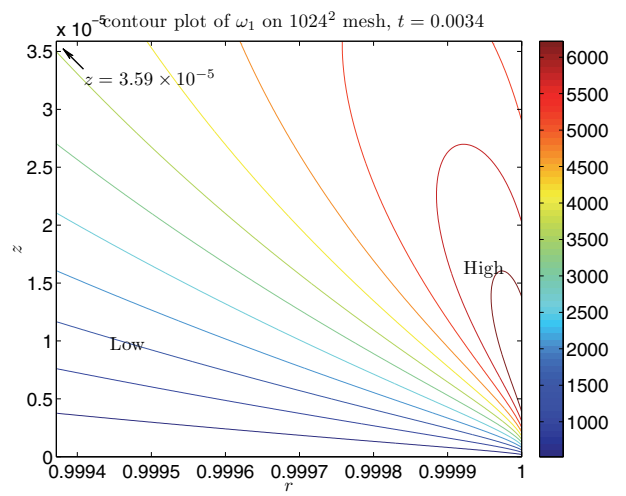

(a) $t=0.0034$

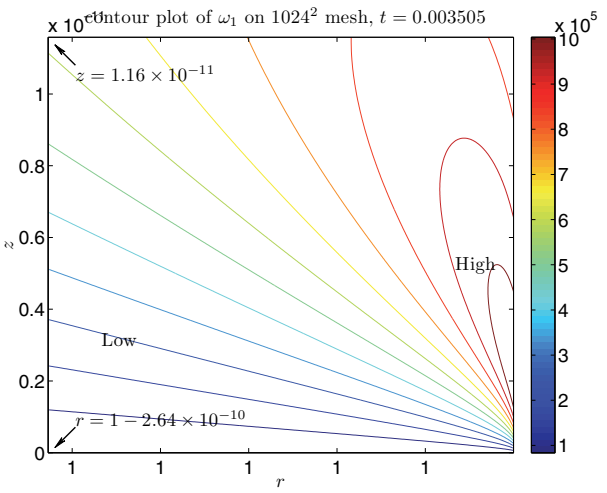

(b) $t=0.003505$

FIG. 15. The $2 D$ contour plot of $\omega_{1}$ near the location of the maximum vorticity at (a) $t=0.0034$ and (b) $t=0.003505$, both computed on the $1024 \times 1024$ mesh.

The rescaled curves $\tilde{C}_{\infty}(t)$, as shown in Figure 13(c), collapse almost perfectly to a single curve, which confirms the existence of a self-similar neighborhood of $\tilde{q}_{0}$. The small variations among the different rescaled curves are shown in Figure 13(d) and are manifestations of the local (inexact) nature of the self-similarity.

4.7.2. Existence of self-similar profiles. By employing a procedure completely similar to that described in the previous section, we examine the solution $\left(u_{1}, \omega_{1}, \psi_{1}\right)$ in the self-similar neighborhood $D_{\infty}(t)$ and confirm the existence of selfsimilar profiles. For purposes of illustration, we plot in Figure 14 the one-dimensional (1D) self-similar profiles of $\omega_{1}$ along selected 1D $r$ - and $z$-mesh lines, and in Figure 15 we plot the $2 \mathrm{D}$ contour plots of $\omega_{1}$ near the location of the maximum vorticity at $t=0.0034$ and 0.003505. Similar plots are also obtained for $u_{1}, \psi_{1}$ and are omitted here for the sake of brevity.

4.7.3. Self-similar analysis. Given the existence of self-similar profiles in the self-similar neighborhood $D_{\infty}(t)$, we conclude that the solution $\left(u_{1}, \omega_{1}, \psi_{1}\right)$ develops 
TABLE 17

Scaling exponents of the self-similar solution (4.17).

\begin{tabular}{cccccccc}
\hline Mesh size & $\hat{\gamma}_{l}$ & $\hat{\gamma}_{u}$ & $\hat{\gamma}_{\omega}$ & $\hat{\gamma}_{\psi}$ & $-1+\frac{1}{2} \hat{\gamma}_{l}$ & $-1+2 \hat{\gamma}_{l}$ & $\hat{\gamma}_{u}-\hat{\gamma}_{l}$ \\
\hline $1024 \times 1024$ & 2.7359 & 0.4614 & -0.9478 & 4.7399 & 0.3679 & 4.4717 & -2.2745 \\
$1280 \times 1280$ & 2.9059 & 0.4629 & -0.9952 & 4.8683 & 0.4530 & 4.8118 & -2.4430 \\
$1536 \times 1536$ & 2.9108 & 0.4600 & -0.9964 & 4.8280 & 0.4554 & 4.8215 & -2.4508 \\
$1792 \times 1792$ & 2.9116 & 0.4602 & -0.9966 & 4.8294 & 0.4558 & 4.8232 & -2.4514 \\
$2048 \times 2048$ & 2.9133 & 0.4604 & -0.9972 & 4.8322 & 0.4567 & 4.8266 & -2.4529 \\
\hline
\end{tabular}

a locally self-similar structure near the point of blowup $\tilde{q}_{0}$. This motivates the representation formula (4.17) with $\tilde{x}_{0}=\tilde{q}_{0}=(1,0)^{T}$. Upon the substitution of (4.17) into the 3D Euler equations (2.1), we obtain the dominant balance

$$
\begin{aligned}
\gamma_{u}-1 & =\gamma_{u}+\gamma_{\psi}-2 \gamma_{l}, \\
\gamma_{\omega}-1 & =\gamma_{\omega}+\gamma_{\psi}-2 \gamma_{l}=2 \gamma_{u}-\gamma_{l}, \\
\gamma_{\psi}-2 \gamma_{l} & =\gamma_{\omega},
\end{aligned}
$$

which, after simplification, yields the one-parameter family of scaling laws

$$
\gamma_{u}=-1+\frac{1}{2} \gamma_{l}, \quad \gamma_{\omega}=-1, \quad \gamma_{\psi}=-1+2 \gamma_{l} .
$$

Table 17 summarizes the scaling exponents estimated from the numerical data. It is clearly seen that (4.20) is approximately satisfied, which confirms the existence of a self-similar solution. In addition to (4.20), it is also known that, for a blowup to occur, the scaling exponent $\gamma_{l}$ must satisfy $\gamma_{l} \geq \frac{2}{5}$ in view of energy conservation [17]. If the velocity $u$ is uniformly bounded, which is the case in our computation, $\gamma_{l}$ must also satisfy $\gamma_{l} \geq 1$ [17]. It is clear from Table 17 that both constraints are satisfied by our numerical data.

The leading-order equations resulting from the dominant balance take the form

$$
\begin{aligned}
-\gamma_{u} U+\gamma_{l}(\tilde{r}, \tilde{z}) \cdot \nabla U+\left[-\Psi_{\tilde{z}} U_{\tilde{r}}+\Psi_{\tilde{r}} U_{\tilde{z}}\right] & =0 \\
-\gamma_{\omega} \Omega+\gamma_{l}(\tilde{r}, \tilde{z}) \cdot \nabla \Omega+\left[-\Psi_{\tilde{z}} \Omega_{\tilde{r}}+\Psi_{\tilde{r}} \Omega_{\tilde{z}}\right] & =2 U U_{\tilde{z}} \\
-\left[\Psi_{\tilde{r} \tilde{r}}+\Psi_{\tilde{z} \tilde{z}}\right] & =\Omega
\end{aligned}
$$

where $(\tilde{r}, \tilde{z})$ are the rescaled coordinates. In principle, one can solve these equations numerically and compare the solutions to that in (4.17), which will confirm the selfsimilar behavior in a different way. This has not been done in the current study but will be considered in a future work.

Finally, the fitting results shown in Table 17 imply that

$$
\begin{gathered}
\omega^{r}=-r u_{1, z}=O\left(t_{s}-t\right)^{-2.45}, \quad \omega^{\theta}=r \omega_{1}=O\left(t_{s}-t\right)^{-1}, \\
\omega^{z}=2 u_{1}+r u_{1, r}=O\left(t_{s}-t\right)^{-2.45},
\end{gathered}
$$

which confirms the scaling law $\|\omega(\cdot, t)\|_{\infty}=O\left(t_{s}-t\right)^{-2.45}$ and hence the existence of a finite-time singularity.

4.8. Understanding the blowup. For the specific initial condition (2.2a) considered in our study, it is observed that $r u_{1}^{0}$ is monotonically increasing in both $r$ and $z$ within the quarter cylinder $D\left(1, \frac{1}{4} L\right)$. It turns out that this property is preserved by the equations (2.1) (for reasons yet to be determined); thus $u_{1, z}$ and consequently 


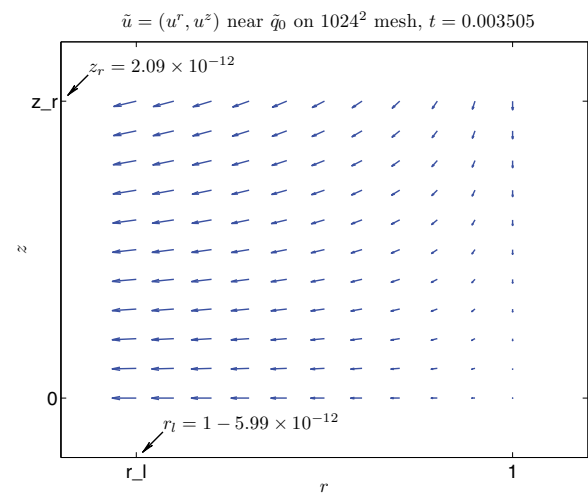

(a) local flow field

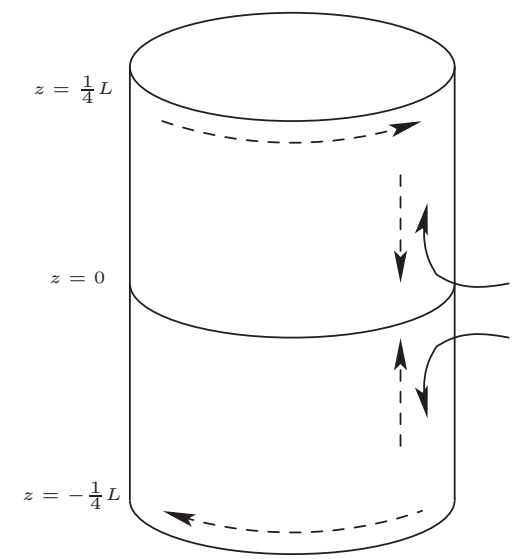

(b) global vorticity dynamics

FIG. 16. Understanding the blowup: (a) local velocity field near the point of the maximum vorticity; (b) global vorticity kinematics of the $3 D$ Euler singularity. In (b), the vortex lines (curved solid arrows) end at the wall and are brought to sections of zero circulation by the axial flow (straight dashed arrows). The curved dash arrows indicate vortical circulation. See also Figure 5 in [14] and Figure 7 in [15].

$\omega_{1}$ (see (2.1b)) remain positive for as long as the solution is smooth. The positivity of $\omega_{1}$ and the homogeneous boundary condition of $\psi_{1}$ together imply the positivity of $\psi_{1}$ (see (2.1c)), which in turn implies that

$$
u^{z}=2 \psi_{1}+r \psi_{1, r}=\psi_{1, r} \leq 0 \quad \text { on } \quad r=1, z \in\left[0, \frac{1}{4} L\right] .
$$

This shows that the flow has a compression mechanism near the corner $\tilde{q}_{0}=(1,0)^{T}$ (Figure 16(a); recall $u^{z}$ is odd at $z=0$ ), which seems to be responsible for the generation of the finite-time singularity observed at $\tilde{q}_{0}$. Indeed, as far as the formation of a singularity is concerned, the precise form of the initial data seems to be immaterial. As long as $r u_{1}^{0}$ has the desired symmetry properties and is monotonically increasing in both $r$ and $z$ in the quarter cylinder $D\left(1, \frac{1}{4} L\right)$, the solution of the initial-boundary value problem (2.1)-(2.2) should develop a singularity in finite time, in much the same way as the solution described in this paper does.

From a physical point of view, the blowup can be deduced from vorticity kinematics applied to the initially rotating eddy [14]. The gradient of circulation down the tube, $2 \pi r u_{z}^{\theta}$, creates a $\theta$-component of vorticity (see $(2.1 \mathrm{~b})$ ). This component in turn creates the flow $\left(u^{r}, u^{z}\right)$ (see (2.1c)-(2.1d)) which advects toward the symmetry plane $z=0$ on the solid wall $r=1$. Since vortex lines threading through the wall are carried by this flow, their points of intersection with the wall move toward the symmetry plane $z=0$ and then collapse onto $z=0$ in finite time (see Figure 16(b)). This is similar to what was observed by [14] in the study of a model problem, which was derived as the leading-order approximation to a stretched version of the TaylorGreen initial value problem for the 3D Euler equations. The model closely resembles the axisymmetric Euler equations, except that the fluid inertia $\left(D_{t} u^{r}\right)$ in the radial transport equation is missing. Since the variable $u^{\theta}$ studied in [14] occurs as coefficients of the asymptotic expansions, the blowup of its $z$-derivatives merely indicates the breakdown of the expansions and the return of the flow to three-dimensionality. It does not imply the loss of regularity of the underlying solutions. 


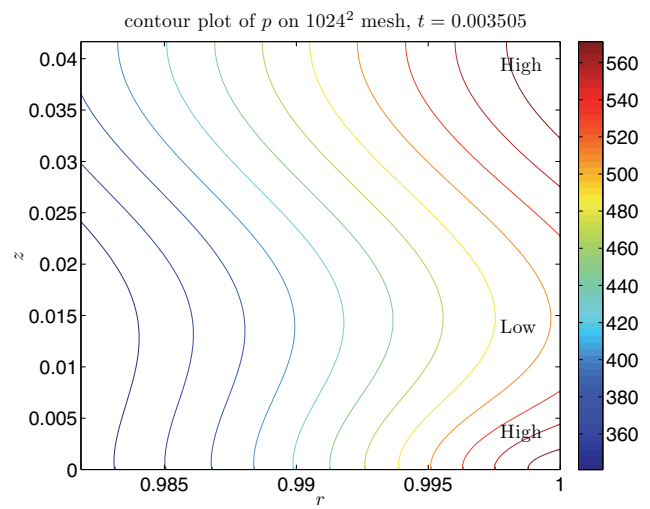

FIG. 17. The contour plot of the pressure $p$ near the location of the maximum vorticity at $t=0.003505$. Note the unfavorable axial pressure gradient near $z=0$.

Despite the apparent similarity between our computations and the model studied by [14], there is a fundamental difference between the two scenarios. More precisely, in [14], it was observed that the absence of radial momentum transfer creates a favorable pressure gradient, which sets up an axial flow near the solid wall toward the symmetry plane $z=0$. In our case, however, it is observed that the pressure gradient near the solid wall $r=1$ and the symmetry plane $z=0$ is unfavorable in the sense that it tends to push fluids away from $z=0$ (Figure 17). Thus unlike the scenario described in [14], it must be the radial fluid inertia, not the pressure gradient, that is responsible for the finite-time blowup observed at the corner $\tilde{q}_{0}=(1,0)^{T}$.

5. Conclusion and future work. In this paper, we have numerically studied the 3D axisymmetric Euler equations in a periodic cylinder and have discovered a class of potentially singular solutions from carefully chosen initial data. By using a specially designed yet highly effective adaptive mesh, we have resolved the nearly singular solution with high accuracy and have advanced the solution to a point asymptotically close to the predicted singularity time. Detailed analysis based on rigorous mathematical blowup/non-blowup criteria, including Beale-Kato-Majda, Constantin-Fefferman-Majda, and Deng-Hou-Yu, provides convincing evidence for the existence of a singularity. Local analysis also suggests the existence of a self-similar blowup in the meridian plane.

Besides providing a promising candidate for the finite-time blowup of the 3D Euler equations, our computations also suggest a possible route to the finite-time blowup of the 2D Boussinesq equations. The Boussinesq equations describe the motion of variable-density, stratified flows under the influence of gravitational forces, and like the 3D Euler equations, the existence or nonexistence of globally regular solutions to the 2D Boussinesq equations is a well-known open problem in fluid dynamics (see, for example, [48]). Since the 2D Boussinesq equations are known to be qualitatively similar to the 3D axisymmetric Euler equations away from the symmetry axis [41], and the singularity discovered in our Euler computations lies on the solid boundary of the cylinder, the solution of the 2D Boussinesq equations resulting from similar initial data is likely to develop a singularity in finite time. This has been confirmed in a separate computation and will be the subject of a forthcoming paper.

Motivated by the observation that the Euler/Boussinesq singularity is likely a 
consequence of a compression flow along the solid wall, we have derived a $1 \mathrm{D}$ model

$$
\begin{aligned}
\theta_{t}+u \theta_{z} & =0, \quad z \in(0, L), \\
\omega_{t}+u \omega_{z} & =\theta_{z},
\end{aligned}
$$

where the nonlocal velocity $u$ is defined by

$$
u(z)=\frac{1}{\pi} \int_{0}^{L} \omega(y) \log |\sin [\mu(z-y)]| d y, \quad \mu=\pi / L .
$$

This 1D model can be viewed as the "restriction" of the 3D axisymmetric Euler equations (2.1) to the wall $r=1$, with the identifications

$$
\theta(z) \sim u_{1}^{2}(1, z), \quad \omega(z) \sim \omega_{1}(1, z), \quad u(z) \sim \psi_{1, r}(1, z) .
$$

We have numerically solved problem (5.1) with the initial condition

$$
u^{0}(z)=10^{4} \sin ^{2}\left(\frac{2 \pi}{L} z\right), \quad \omega^{0}(z)=0,
$$

and discovered that the solution develops a singularity in finite time in much the same way as the solution to the 3D Euler equations (2.1) does (Figure 18). The details of these computations, as well as the derivation and analysis of the 1D model (5.1), will be reported in a forthcoming paper.

Appendix A. Construction of the adaptive mesh. The mesh mapping functions $r(\rho), z(\eta)$ are defined through an analytic function $\mu$,

$$
r(\rho)=\mu\left(\rho ; \alpha^{r}, \sigma^{r}\right), \quad z(\eta)=\mu\left(\eta ; \alpha^{z}, \sigma^{z}\right),
$$

where $\alpha^{r}, \sigma^{r}$, etc., are parameters and

$$
\mu_{s}(s ; \alpha, \sigma)=\alpha_{0}+\alpha_{1} \mathrm{e}^{-\pi s^{2} / \sigma_{1}^{2}}+\alpha_{2} \mathrm{e}^{-\pi(s-1)^{2} / \sigma_{2}^{2}}, \quad s \in[0,1] .
$$

The particular form of $\mu$ is chosen to meet the following goals. First, it should map the interval $[0,1]$ onto another interval, say $[0, L]$, in a one-to-one manner. Second, given any subset $[a, b]$ of $[0, L]$ and any $\delta \in(0,1)$, it should place at least a $\delta$-fraction of the mesh points in $[a, b]$ and maintain a uniform mesh on $[a, b]$. In our computations, the interval $[0, L]$ will be the entire computational domain along either the $r$ - or the $z$-dimension, and $[a, b]=\mathcal{P}\left\{(r, z):|\omega| \geq \delta_{0}\|\omega\|_{\infty}\right\}$ will be a small neighborhood of the maximum vorticity along that dimension where $\mathcal{P}$ is the projection to $r$ or to $z$ and $\delta_{0} \in(0,1)$ is a small parameter. The mesh mapping functions constructed in this way will always place enough points near the maximum vorticity, provided that the vorticity blows up in a self-similar fashion with a bell-shaped similarity profile. This is what we observe in our case.

The one-to-one correspondence of the map generated by $\mu$ is equivalent to the positivity of $\mu_{s}$, which can be ensured, provided that $\alpha_{0}>0$ and $\alpha_{1}, \alpha_{2} \geq 0$. To place the required amount of mesh points in the interval $[a, b]$ and ensure a uniform mesh on $[a, b]$, we observe that

$$
\mu_{s}(s ; \alpha, \sigma)=\alpha_{0}+\alpha_{1} \mathrm{e}^{-\pi s^{2} / \sigma_{1}^{2}}+\alpha_{2} \mathrm{e}^{-\pi(s-1)^{2} / \sigma_{2}^{2}} \approx \alpha_{0}, \quad s \in\left[2 \sigma_{1}, 1-2 \sigma_{2}\right],
$$




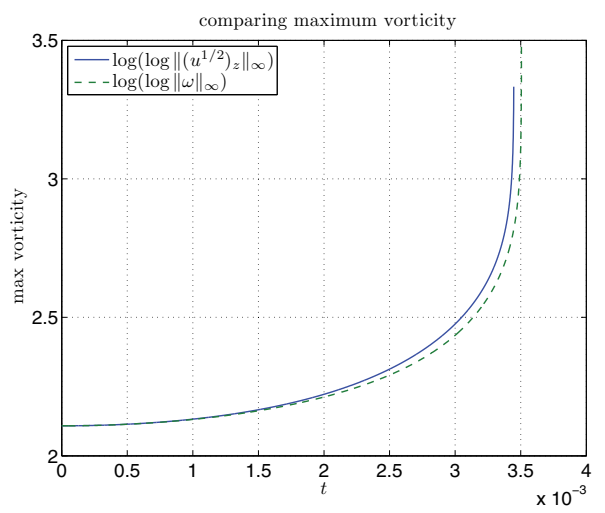

(a) $\log \left(\log \left\|\left(u^{1 / 2}\right)_{z}\right\|_{\infty}\right)$ vs. $\log \left(\log \|\omega\|_{\infty}\right)$

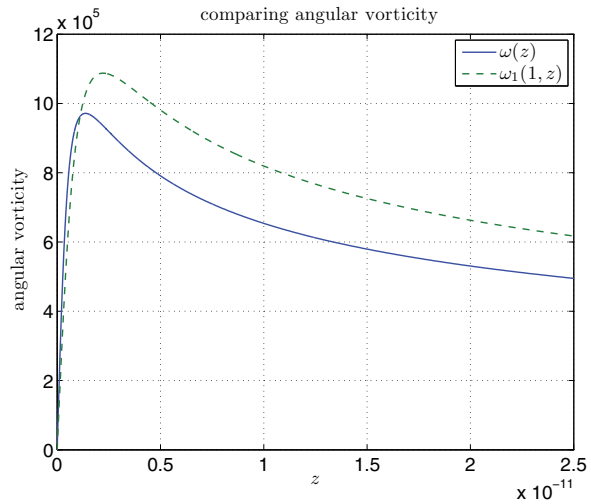

(c) $\omega(z)$ vs. $\omega_{1}(1, z)$

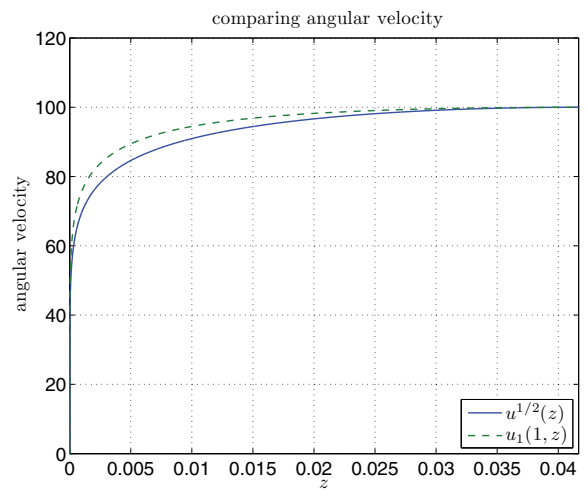

(b) $u^{1 / 2}(z)$ vs. $u_{1}(1, z)$

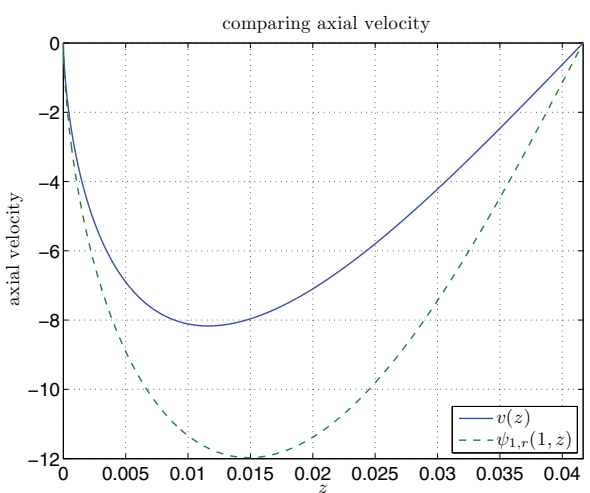

(d) $v(z)$ vs. $\psi_{1, r}(1, z)$

FIG. 18. Comparison of numerical solutions of the $1 D$ model (5.1) with those of the $3 D$ axisymmetric Euler equations (2.1): (a) maximum vorticity, (b) angular velocity, (c) angular vorticity, and (d) axial velocity. In all the plots the solution of the $1 D$ model is computed at $t=0.003447$ and that of the $3 D$ Euler is computed at $t=0.003505$.

in view of the rapid decay of the Gaussians away from their centers. Therefore, if we choose $\left(\sigma_{1}, \sigma_{2}\right)$ such that $1-2 \sigma_{1}-2 \sigma_{2}=\delta$ and map the interval $\left[2 \sigma_{1}, 1-2 \sigma_{2}\right]$ onto $[a, b]$, the resulting mesh will have the desired properties.

We remark that there are other ways to construct adaptive meshes with similar point concentration properties. Examples include the popular sine transform

$$
\mu(s)=s+\frac{\alpha}{\pi} \sin (\pi s), \quad s \in[0,1], \alpha \in(-1,1),
$$

the Chebyshev (cosine) transform

$$
\mu(s)=\cos (s), \quad s \in[0, \pi],
$$

and the "negative Gaussian" transform

$$
\mu(s)=s-\alpha \mathrm{e}^{-\left(s-s_{0}\right)^{2} / \sigma^{2}}, \quad s \in[0,1], \alpha>0 .
$$

The drawback of these "traditional" mapping functions is that the resulting mesh has unlimited resolution at only a single point, and this point must be one of the 
end points in the case of sine/cosine transforms. This is inadequate when unlimited resolution is demanded in an interval, such as in our case, and the mapping function proposed in (A.1) provides an effective way to achieve this.

The mapping function $\mu$ defined by (A.1) is constructed using the following procedure. First, the parameters $\left(\sigma_{1}, \sigma_{2}\right)$, which specify the amount of points to be distributed to the intervals $[0, a]\left(2 \sigma_{1}\right),[a, b]\left(1-2 \sigma_{1}-2 \sigma_{2}\right)$, and $[b, L]\left(2 \sigma_{2}\right)$, are supplied by the users and are fixed throughout the computations. To ensure a proper mesh, these parameters must satisfy

$$
0<\sigma_{1}, \sigma_{2}<\frac{1}{4} .
$$

Next, the parameters $\left(\alpha_{0}, \alpha_{1}, \alpha_{2}\right)$ are determined from the equations

$$
\mu(0)=0, \quad \mu\left(2 \sigma_{1}\right)=a, \quad \mu\left(1-2 \sigma_{2}\right)=b, \quad \mu(1)=L,
$$

which ensure that $[0,1]$ is mapped onto $[0, L]$ and $\left[2 \sigma_{1}, 1-2 \sigma_{2}\right]$ is mapped onto $[a, b]$. If $\left(\sigma_{1}, \sigma_{2}\right)$ are reasonably small, as we shall assume in what follows, (A.2b) may be replaced by the approximate equations

$$
\begin{aligned}
2 \sigma_{1} \alpha_{0}+\frac{1}{2} \sigma_{1} \alpha_{1} & =a, \\
\left(1-2 \sigma_{2}\right) \alpha_{0}+\frac{1}{2} \sigma_{1} \alpha_{1} & =b, \\
\alpha_{0}+\frac{1}{2} \sigma_{1} \alpha_{1}+\frac{1}{2} \sigma_{2} \alpha_{2} & =L,
\end{aligned}
$$

which can be readily solved to give

$$
\alpha_{0}=\frac{b-a}{1-2 \sigma_{1}-2 \sigma_{2}}, \quad \alpha_{1}=\frac{2}{\sigma_{1}}\left(a-2 \sigma_{1} \alpha_{0}\right), \quad \alpha_{2}=\frac{2}{\sigma_{2}}\left(L-b-2 \sigma_{2} \alpha_{0}\right) .
$$

Note that $\alpha_{0}>0$, since $b>a$ (by construction) and $\sigma_{1}+\sigma_{2}<\frac{1}{2}$ (by constraint (A.2a)). If $\alpha_{1}$ and $\alpha_{2}$ as given by (A.3) are both nonnegative, then a unique, strictly increasing mesh mapping function $\mu$ satisfying (A.2) results. If not, then the values of $\alpha_{i}$ 's need to be adjusted to maintain the strict monotonicity of $\mu$. Consider first the case where $\alpha_{1}$ as given by (A.3) is negative. In this case the left end point $a$ of the "singularity interval" $[a, b]$ is too close to $\mu=0$ (so close that $a<2 \sigma_{1} \alpha_{0}$ ), and the interval $[0, a]$ must be merged with $[a, b]$ to form a larger singularity interval $[0, b]$. The mesh mapping function is modified accordingly by setting $\alpha_{1}=0$ in (A.1):

$$
\mu_{s}(s ; \alpha, \sigma)=\alpha_{0}+\alpha_{2} \mathrm{e}^{-\pi(s-1)^{2} / \sigma_{2}^{2}}, \quad s \in[0,1],
$$

and the values of $\alpha_{0}, \alpha_{2}$ are recomputed from the constraints:

$$
\mu\left(1-2 \sigma_{2}\right)=b, \quad \mu(1)=L,
$$

which yield, after suitable simplifications,

$$
\alpha_{0}=\frac{b}{1-2 \sigma_{2}}>0, \quad \alpha_{2}=\frac{2}{\sigma_{2}}\left(L-\alpha_{0}\right) .
$$

If $\alpha_{2}$ computed in this way is still negative, then the right end point $b$ of the (extended) singularity interval is too close to $\mu=L$ (so close that $\left(1-2 \sigma_{2}\right) L<b$ ), and the interval $[b, L]$ must be merged with $[0, b]$ to form a larger singularity interval $[0, L]$. In this case the mesh mapping function simply takes the form

$$
\mu_{s}(s ; \alpha, \sigma)=\alpha_{0}=L, \quad s \in[0,1],
$$


and the adaptive mesh reduces to a uniform mesh. The case where $\alpha_{1} \geq 0, \alpha_{2}<0$ in (A.3) can be handled in a similar way.

Appendix B. Construction of the B-spline subspace. Consider the finitedimensional subspace of weighted uniform B-splines of even order $k$ :

$$
V_{h}:=V_{w, h}^{k}=\operatorname{span}\left\{w(\rho) b_{j, h_{r}}^{k}(\rho) b_{i, h_{z}}^{k}(\eta)\right\} \cap V,
$$

where $w(\rho)=1-\rho^{2}, h_{r}=1 / N, h_{z}=1 / M$, and

$$
\begin{aligned}
V=\operatorname{span}\left\{\phi \in H^{1}[0,1]^{2}: \phi(-\rho, \eta)\right. & =\phi(\rho, \eta), \\
\phi(1, \eta) & =0, \phi(\rho, \ell-\eta)=-\phi(\rho, \ell+\eta) \forall \ell \in \mathbb{Z}\} .
\end{aligned}
$$

The functions $b_{\ell, h}^{k}(s)=b^{k}((s / h)-(\ell-k / 2))$ are shifted and rescaled uniform B-splines of order $k$ where $b^{k}$, the "reference" uniform B-splines, satisfy the recursion [30]

$$
\begin{gathered}
b^{1}(s)=\chi_{[0,1)}(s)= \begin{cases}1 & \text { if } 0 \leq s<1, \\
0 & \text { otherwise }\end{cases} \\
b^{k}(s)=\int_{s-1}^{s} b^{k-1}(\tau) d \tau, \quad k \geq 2 .
\end{gathered}
$$

A basis of the subspace $V_{h}$ can be conveniently chosen as

$$
B_{w, i, j}(\rho, \eta):=w(\rho) B_{j}(\rho) B_{i}(\eta), \quad 1 \leq i \leq M-1,0 \leq j \leq N+k / 2-1,
$$

where

$$
B_{j}(\rho)=\frac{b_{j, h_{r}}^{k}(\rho)+b_{j, h_{r}}^{k}(-\rho)}{1+\delta_{j 0}}, \quad B_{i}(\eta)=\sum_{\ell=-\infty}^{\infty}\left[b_{i, h_{z}}^{k}(2 \ell+\eta)-b_{i, h_{z}}^{k}(2 \ell-\eta)\right] .
$$

If we write

$$
\psi_{h}(\rho, \eta)=\sum_{i, j} c_{i j} B_{w, i, j}(\rho, \eta)
$$

then the finite-dimensional variational problem

$$
a\left(\psi_{h}, \phi_{h}\right)=f\left(\phi_{h}\right) \quad \forall \phi_{h} \in V_{h}
$$

can be transformed to an equivalent linear system $A x=b$, which in component form reads as

$$
\sum_{i, j} a\left(B_{w, i, j}, B_{w, m, n}\right) c_{i j}=f\left(B_{w, m, n}\right)
$$

In our computations, the entries of $A, b$ are approximated using composite 6-point Gauss-Legendre quadrature rules. This essentially reproduces the exact values of the stiffness matrix $A$ and hence ensures the uniform $V_{h}$-ellipticity of the approximate bilinear forms and the convergence of the discrete approximations [16]. The large sparse linear system resulting from the Galerkin discretization is solved using the 
PaStiX package, ${ }^{11}$ a parallel sparse direct solver based on the supernodal (left-looking) method [29].

Appendix C. Description of the test problem. The finite-element, finitedifference hybrid adaptive method described in section 3 is applied to a forced axisymmetric Euler system:

$$
\begin{aligned}
u_{1, t}+u^{r} u_{1, r}+u^{z} u_{1, z} & =2 u_{1} \psi_{1, z}+F^{u}, \\
\omega_{1, t}+u^{r} \omega_{1, r}+u^{z} \omega_{1, z} & =\left(u_{1}^{2}\right)_{z}+F^{\omega}, \\
-\left[\partial_{r}^{2}+(3 / r) \partial_{r}+\partial_{z}^{2}\right] \psi_{1} & =\omega_{1},
\end{aligned}
$$

where the forcing terms $F^{u}, F^{\omega}$ are generated from a smooth test solution:

$$
\begin{aligned}
\tilde{u}_{1}(r, z, t) & =\xi(r, T-t) \sin \left[\frac{1}{2} \pi \zeta(z, T-t)\right], \\
\tilde{\psi}_{1}(r, z, t) & =30\left(1-r^{2}\right) \xi(r, T-t) \sin [\pi \zeta(z, T-t)], \\
\tilde{\omega}_{1}(r, z, t) & =-\left[\partial_{r}^{2}+(3 / r) \partial_{r}+\partial_{z}^{2}\right] \tilde{\psi}_{1}(r, z, t) .
\end{aligned}
$$

The solution as given by (C.2) develops a singularity at a finite time $T$ with locally self-similar profiles determined by the functions $\xi$, $\zeta$, which in our case are chosen to be

$$
\xi(r, t)=t^{2} \exp \left(-\frac{1-r^{2}}{10 t^{2}}\right), \quad \zeta(z, t)=\tanh \left(\frac{2 z}{5 L t^{2}}\right) .
$$

The velocity component $\tilde{u}_{1}$ of the test solution contains a sharp front near $\tilde{q}_{0}=$ $(1,0)^{T}$, which would become a shock with finite strength at $t=T$ if the scaling factor $t^{2}$ in $\xi(r, t)$ were absent (this scaling factor is introduced to mitigate the stiff forcing terms $\left.F^{u}, F^{\omega}\right)$. Meanwhile, the vorticity component $\tilde{\omega}_{1}$ contains a sharp peak propagating toward $\tilde{q}_{0}$, which would blow up at $t=T$ without the $t^{2}$ factor. This particular test solution closely resembles the behavior of the potentially singular Euler solution computed from (2.1)-(2.2), and it provides an excellent benchmark on the performance of the numerical method described in section 3.

The forced system (C.1) is complemented with the initial condition

$$
u_{1}^{0}(r, z)=\tilde{u}_{1}(r, z, 0), \quad \omega_{1}^{0}(r, z)=\tilde{\omega}_{1}(r, z, 0), \quad \psi_{1}^{0}(r, z)=\tilde{\psi}_{1}(r, z, 0)
$$

and boundary conditions $(2.2 \mathrm{~b})-(2.2 \mathrm{c})$. It is solved with $T=0.03, L=\frac{1}{6}$ on the quarter cylinder $D\left(1, \frac{1}{24}\right)$ to $t=0.029$, at which time the errors are computed and reported in Table 7.

Appendix D. Derivation of the lower bound for $\boldsymbol{K}_{\rho}\left(q_{0}\right)$. Consider the quantity

$$
K_{\rho}\left(q_{0}, t\right)=\int_{|y| \leq \rho}\left|D\left(\hat{y}, \xi\left(q_{0}+y, t\right), \xi\left(q_{0}, t\right)\right)\right| \cdot\left|\omega\left(q_{0}+y, t\right)\right| \frac{d y}{|y|^{3}} .
$$

To obtain a lower bound for the above integral, we consider the set $N_{0, t}=V_{0, t}-q_{0}$, where

$$
\begin{gathered}
V_{0, t}=\left\{\left(x_{1}, x_{2}, x_{3}\right) \in \mathbb{R}^{3}:\left(\sqrt{x_{1}^{2}+x_{2}^{2}}, x_{3}\right) \in D_{\infty}(t), \tan ^{-1}\left(x_{2} / x_{1}\right) \in\left(-d_{1}, 0\right)\right\}, \\
d_{1}=d_{1}\left(D_{\infty}(t)\right)=\min _{\tilde{y} \in C_{\infty}(t)}\left|\tilde{y}-\tilde{q}_{0}\right|
\end{gathered}
$$

\footnotetext{
${ }^{11}$ https://gforge.inria.fr/projects/pastix
} 
where $C_{\infty}(t), D_{\infty}(t)$ are as defined in (4.18) and (4.12). In words, $V_{0, t}$ is the "cylindrical shell" obtained by rotating the set $D_{\infty}(t)$ about the symmetry axis $r=0$, starting from the angle $\theta=0$ and ending at the angle $\theta=-d_{1}$. Since the diameter of $D_{\infty}(t)$ shrinks rapidly to 0 as $t$ approaches $t_{s}$, we deduce that $N_{0, t} \subseteq B_{\rho}(0)$ for $t$ sufficiently close to $t_{s}$, and hence (recall $|\omega| \geq \frac{1}{2}\|\omega\|_{\infty}$ on $D_{\infty}(t)$ )

$$
K_{\rho}\left(q_{0}, t\right) \geq \frac{1}{2}\|\omega(\cdot, t)\|_{\infty} \int_{N_{0, t}}\left|D\left(\hat{y}, \xi\left(q_{0}+y, t\right), \xi\left(q_{0}, t\right)\right)\right| \frac{d y}{|y|^{3}} .
$$

To continue, we observe that

$$
\omega^{\theta}=r \omega_{1}=0, \quad \omega^{z}=2 u_{1}+r u_{1, r}=0 \quad \text { on } \quad z=0,
$$

due to the odd symmetry of $u_{1}, \omega_{1}$ at $z=0$ (see section 2). This means that the direction vectors $\xi\left(q_{0}+y\right)$, when restricted to the plane $z=0$, all point in the radial direction $-(\cos \theta, \sin \theta, 0)^{T}$ and hence are closely aligned with $\xi\left(q_{0}\right)=(-1,0,0)^{T}$, provided that $|\theta| \ll 1$. Consequently, $|D|$ is small near the intersection of the planes $z=0$ and $\theta=0$. In addition, for a point $x=(\cos \theta, \sin \theta, z)^{T}$ lying on the solid wall $r=1$, the vector $y=x-q_{0}$ satisfies

$$
\hat{y} \cdot \xi\left(q_{0}\right)=\frac{(\cos \theta-1, \sin \theta, z)}{|(\cos \theta-1, \sin \theta, z)|} \cdot(-1,0,0)=\frac{1-\cos \theta}{\sqrt{2-2 \cos \theta+z^{2}}} \approx \frac{\theta^{2}}{2 \sqrt{\theta^{2}+z^{2}}},
$$

provided that $|\theta| \ll \min \{|z|, 1\}$. This shows that $\hat{y} \cdot \xi\left(q_{0}\right)$ and hence $|D|$ is small near the intersection of the wall $r=1$ and the plane $\theta=0$. Motivated by these observations, we choose to estimate $|D|$ on the set $N_{1, t}=V_{1, t}-q_{0} \subseteq N_{0, t}$, where

$$
\begin{gathered}
V_{1, t}=\left\{\left(x_{1}, x_{2}, x_{3}\right) \in V_{0, t}:\left(\sqrt{x_{1}^{2}+x_{2}^{2}}, x_{3}\right) \in S_{t}+\tilde{q}_{0}\right\}, \\
S_{t}=\left\{\left(\tilde{y}_{1}, \tilde{y}_{2}\right): \phi=\tan ^{-1}\left(\tilde{y}_{2} / \tilde{y}_{1}\right) \in\left(\frac{11}{20} \pi, \frac{3}{4} \pi\right),|\tilde{y}| \in\left(\frac{1}{2} \rho(\phi), \rho(\phi)\right)\right\},
\end{gathered}
$$

where $\rho(\phi), \phi \in\left[\frac{1}{2} \pi, \pi\right]$, denotes a parametrization of the curve $C_{\infty}(t)$ in polar coordinates (see Figure 13(a) for an illustration of $C_{\infty}(t)$ ). Note that $S_{t}+\tilde{q}_{0}$ lies in the interior of the set $D_{\infty}(t)$ and stays away from the rays $z=0, r=1$ where $|D|$ is small.

Now we estimate

$$
K_{\rho}\left(q_{0}, t\right) \geq \frac{1}{2}\|\omega(\cdot, t)\|_{\infty} \ell_{D, q_{0}}(t) \int_{N_{1, t}} \frac{d y}{|y|^{2}}
$$

where

$$
\ell_{D, q_{0}}(t)=\inf _{y \in N_{1, t}} \frac{1}{|y|}\left|D\left(\hat{y}, \xi\left(q_{0}+y, t\right), \xi\left(q_{0}, t\right)\right)\right| .
$$

For each $y \in N_{1, t}$ and $x \in V_{1, t}$ such that $y=x-q_{0}$, we have

$$
|y|=\left|x-q_{0}\right| \leq|x-\tilde{x}|+\left|\tilde{x}-\tilde{q}_{0}\right| \leq d_{1}+\rho(\phi) \leq 2 \rho(\phi), \quad \phi=\pi+\tan ^{-1} \frac{x_{3}}{r-1},
$$

where as usual $\tilde{x}=\left(r, x_{3}\right)^{T}$ denotes the projection of $x$ onto the $r z$-plane. It then follows that

$$
\int_{N_{1, t}} \frac{d y}{|y|^{2}} \geq \frac{1}{4} \int_{N_{1, t}} \frac{d y}{\rho^{2}(\phi)} \geq \frac{1}{8} d_{1} \int_{11 \pi / 20}^{3 \pi / 4} \rho^{-2}(\phi) \int_{\rho(\phi) / 2}^{\rho(\phi)} s d s d \phi=\frac{3}{320} d_{1} \pi
$$

Copyright $\odot$ by SIAM. Unauthorized reproduction of this article is prohibited. 
where in the second inequality above we have used the fact that, for any $y \in N_{1, t}$, the distance between the point $x=y+q_{0}$ and the symmetry axis is greater than $\frac{1}{2}$ for $t$ sufficiently close to $t_{s}$. This leads to the estimate (4.13a):

$$
K_{\rho}\left(q_{0}, t\right) \geq \frac{3 \pi}{640} d_{1}\left(D_{\infty}(t)\right)\|\omega(\cdot, t)\|_{\infty} \ell_{D, q_{0}}(t)
$$

Acknowledgments. This research was supported in part by NSF FRG grant DMS-1159138 and DOE grant DE-FG02-06ER25727. The authors would like to gratefully acknowledge the computing resources provided by the SHC cluster at Caltech Center for Advanced Computing Research (CACR) and the Brutus cluster at ETH Zürich (ETHZ). The authors gratefully acknowledge the excellent support provided by the staff members at SHC, especially Sharon Brunett, and the support provided by Prof. Petros Koumoutsakos at ETHZ, who kindly allowed them to use his computing resources. The authors also thank the anonymous referees for their helpful comments. The first author gratefully acknowledges the travel support provided by NSF FRG grant DMS-1159133, made available to him by Prof. Alexander Kiselev, for his trip to 2013 Stanford summer school, and by the Department of Computing and Mathematical Sciences at Caltech for his trip to 2013 AMS Fall central sectional meeting at Washington University in St. Louis.

\section{REFERENCES}

[1] M. Arioli, J. W. Demmel, And I. S. Duff, Solving sparse linear systems with sparse backward error, SIAM J. Matrix Anal. Appl., 10 (1989), pp. 165-190.

[2] C. Bardos and E. S. Titi, Euler equations for incompressible ideal fluids, Russian Math. Surveys, 62 (2007), pp. 409-451.

[3] J. T. Beale, T. Kato, And A. Majda, Remarks on the breakdown of smooth solutions for the 3-D Euler equations, Comm. Math. Phys., 94 (1984), pp. 61-66.

[4] M. Berger And R. V. Kohn, A rescaling algorithm for the numerical calculation of blowing-up solutions, Comm. Pure Appl. Math., 41 (1988), pp. 841-863.

[5] O. N. Boratav and R. B. Pelz, Direct numerical simulation of transition to turbulence from a high-symmetry initial condition, Phys. Fluids, 6 (1994), pp. 2757-2784.

[6] C. J. Budd, S. Chen, ANd R. D. Russell, New self-similar solutions of the nonlinear Schrödinger equation with moving mesh computations, J. Comput. Phys., 152 (1999), pp. $756-789$.

[7] C. J. Budd, W. Huang, And R. D. Russell, Moving mesh methods for problems with blow-up, SIAM J. Sci. Comput., 17 (1996), pp. 305-327.

[8] M. D. Bustamante And R. M. KerR, 3D Euler about a 2D symmetry plane, Phys. D, 237 (2008), pp. 1912-1920.

[9] L. Caffarelli, R. Kohn, and L. Nirenberg, Partial regularity of suitable weak solutions of the Navier-Stokes equations, Comm. Pure Appl. Math., 35 (1982), pp. 771-831.

[10] R. E. CAflisch, Singularity formation for complex solutions of the $3 D$ incompressible Euler equations, Phys. D, 67 (1993), pp. 1-18.

[11] D. CHAE, Nonexistence of self-similar singularities for the 3D incompressible Euler equations, Comm. Math. Phys., 273 (2007), pp. 203-215.

[12] D. CHAE, On the generalized self-similar singularities for the Euler and the Navier-Stokes equations, J. Funct. Anal., 258 (2010), pp. 2865-2883.

[13] D. Chat, On the self-similar solutions of the 3D Euler and the related equations, Comm. Math. Phys., 305 (2011), pp. 333-349.

[14] S. Childress, Nearly two-dimensional solutions of Euler's equations, Phys. Fluids, 30 (1987), pp. 944-953.

[15] S. Childress, Topological Fluid Dynamics for Fluid Dynamicists, preprint, 2004.

[16] P. G. Ciarlet, The Finite Element Method for Elliptic Problems, Classics Appl. Math. 40, SIAM, Philadelphia, 2002.

[17] P. Constantin, Geometric statistics in turbulence, SIAM Rev., 36 (1994), pp. 73-98.

[18] P. Constantin, On the Euler equations of incompressible fluids, Bull. Amer. Math. Soc. (N.S.), 44 (2007), pp. 603-621. 
[19] P. Constantin, C. Fefferman, and A. Majda, Geometric constraints on potentially singular solutions for the 3-D Euler equations, Comm. Partial Differential Equations, 21 (1996), pp. 559-571.

[20] J. Deng, T. Y. Hou, And X. Yu, Geometric properties and non-blowup of $3 D$ incompressible Euler flow, Comm. Partial Differential Equations, 30 (2005), pp. 225-243.

[21] W. E And C.-W. Shu, Small-scale structures in Boussinesq convection, Phys. Fluids, 6 (1994), pp. 49-58.

[22] A. B. Ferrari, On the blow-up of solutions of the 3-D Euler equations in a bounded domain, Comm. Math. Phys., 155 (1993), pp. 277-294.

[23] H. Fujita, On the blowing up of solutions of the Cauchy problem for $u_{t}=\Delta u+u^{1+\alpha}$, J. Fac. Sci. Univ. Tokyo Sect. A Math., 16 (1966), pp. 105-113.

[24] J. D. GibBon, The three-dimensional Euler equations: Where do we stand?, Phys. D, 237 (2008), pp. 1894-1904.

[25] J. D. Gibbon And E. S. Titi, The 3D incompressible Euler equations with a passive scalar: A road to blow-up?, J. Nonlinear Sci., 23 (2013), pp. 993-1000.

[26] R. Grauer, C. Marliani, and K. Germaschewski, Adaptive mesh refinement for singular solutions of the incompressible Euler equations, Phys. Rev. Lett., 80 (1998), pp. 4177-4180.

[27] R. Grauer and T. C. Sideris, Numerical computation of 3D incompressible ideal fluids with swirl, Phys. Rev. Lett., 67 (1991), pp. 3511-3514.

[28] B. Gustafsson, H.-O. Kreiss, and J. Oliger, Time Dependent Problems and Difference Methods, Wiley-Interscience, New York, 1995.

[29] P. HÉnon, P. Ramet, and J. Roman, PaStiX: A high-performance parallel direct solver for sparse symmetric definite systems, Parallel Comput., 28 (2002), pp. 301-321.

[30] K. HöLlig, Finite Element Methods with B-Splines, SIAM, Philadelphia, 2003.

[31] T. Y. Hou AND Z. LEI, On the stabilizing effect of convection in three-dimensional incompressible flows, Comm. Pure Appl. Math., 62 (2009), pp. 501-564.

[32] T. Y. Hou And R. LI, Dynamic depletion of vortex stretching and non-blowup of the 3-D incompressible Euler equations, J. Nonlinear Sci., 16 (2006), pp. 639-664.

[33] T. Y. Hou AND R. Li, Blowup or no blowup? The interplay between theory and numerics, Phys. D, 237 (2008), pp. 1937-1944.

[34] W. Huang, J. Ma, and R. D. Russell, A study of moving mesh PDE methods for numerical simulation of blowup in reaction diffusion equations, J. Comput. Phys., 227 (2008), pp. 6532-6552.

[35] W. Huang, Y. Ren, And R. D. Russell, Moving mesh methods based upon moving mesh partial differential equations, J. Comput. Phys., 113 (1994), pp. 279-290.

[36] Y. Huang And A. Bertozzi, Self-similar blowup solutions to an aggregation equation in $R^{n}$, SIAM J. Appl. Math., 70 (2010), pp. 2582-2603.

[37] T. KATO, Nonstationary flows of viscous and ideal fluids in $R^{3}$, J. Funct. Anal., 9 (1972), pp. 296-305.

[38] R. M. KERR, Evidence for a singularity of the three-dimensional incompressible Euler equations, Phys. Fluids A, 5 (1993), pp. 1725-1746.

[39] R. M. KerR, Bounds for Euler from vorticity moments and line divergence, J. Fluid Mech., 729 (2013), R2.

[40] J.-G. LIU AND W.-C. WANG, Convergence analysis of the energy and helicity preserving scheme for axisymmetric flows, SIAM J. Numer. Anal., 44 (2006), pp. 2456-2480.

[41] A. Majda And A. Bertozzi, Vorticity and Incompressible Flow, Cambridge University Press, Cambridge, UK, 2002.

[42] D. W. Mclaughlin, G. C. Papanicolaou, C. Sulem, and P. L. Sulem, Focusing singularity of the cubic Schrödinger equation, Phys. Rev. A, 34 (1986), pp. 1200-1210.

[43] P. Orlandi and G. F. Carnevale, Nonlinear amplification of vorticity in inviscid interaction of orthogonal Lamb dipoles, Phys. Fluids, 19 (2007), 057106.

[44] G. Ponce, Remarks on a paper by J. T. Beale, T. Kato, and A. Majda, Comm. Math. Phys., 98 (1985), pp. 349-353.

[45] A. Pumir And E. D. Siggia, Development of singular solutions to the axisymmetric Euler equations, Phys. Fluids A, 4 (1992), pp. 1472-1491.

[46] T. Shirota And T. Yanagisawa, A continuation principle for the 3-D Euler equations for incompressible fluids in a bounded domain, Proc. Japan Acad. Ser. A Math. Sci., 69 (1993), pp. $77-82$.

[47] M. Siegel And R. E. Caflisch, Calculation of complex singular solutions to the $3 D$ incompressible Euler equations, Phys. D, 238 (2009), pp. 2368-2379.

[48] V. I. Yudovich, Eleven great problems of mathematical hydrodynamics, Mosc. Math. J., 3 (2003), pp. 711-737.

Copyright (c) by SIAM. Unauthorized reproduction of this article is prohibited. 